Final accepted version of : Moradi, G., Vermeulen, B., Rennie, C., Cardot, R. and Lane, S.N., 2019..

Evaluation of aDcp processing options for secondary flow identification at river junctions. Earth Surface Processes and Landforms. Fully formatted final version is freely available as Read Only at https://rdcu.be/bVZNP

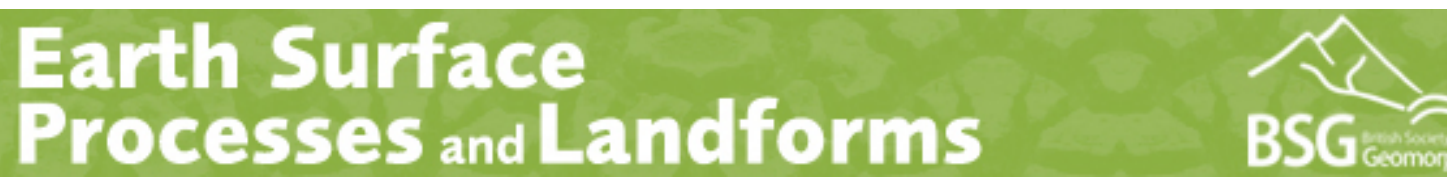

\title{
Evaluation of aDcp processing options for secondary flow identification at river junctions
}

\begin{tabular}{|r|l|}
\hline Journal: & Earth Surface Processes and Landforms \\
\hline Manuscript ID & ESP-19-0030.R2 \\
\hline Diley - Manuscript type: & Research Article \\
\hline Author: & $31-$ Jul-2019 \\
\hline & $\begin{array}{l}\text { Complete List of Authors: } \\
\text { Geosciences and Environment } \\
\text { Water Engineering and Management Department } \\
\text { Rennie, Colin; University of Ottawa, Civil Engineering } \\
\text { Cardot, Romain; UNIL, Institute of Earth Surface Dynamics, Faculty of } \\
\text { Geosciences and Environment } \\
\text { Lane, Stuart; UNIL, Institute of Earth Surface Dynamics, Faculty of } \\
\text { Geosciences and Environment }\end{array}$ \\
\hline Keywords: & $\begin{array}{l}\text { Acoustic Doppler current profiler, Secondary circulation, River } \\
\text { confluences, River junctions }\end{array}$ \\
\hline \multicolumn{2}{|l}{} \\
\hline You must view these files (e.g. movies) online.
\end{tabular}

\section{SCHOLARONE Manuscripts}


1 "For the ESPL special issue: Measuring and numerical modelling of hydro-

2 morphological processes in open-water"

3 Evaluation of aDcp processing options for secondary flow 4 identification at river junctions

5 Gelare Moradi ${ }^{1}$, Bart Vermeulen ${ }^{3}$, Colin D. Rennie ${ }^{2}$, Romain Cardot ${ }^{1}$, Stuart N. Lane ${ }^{1}$

6 1: University of Lausanne - Institute of Earth Surface Dynamics (UNIL - IDYST)

7 Université de Lausanne - IDYST Quartier Mouline - Bâtiment Géopolis - Switzerland

8 2: Civil Engineering Department, University of Ottawa

9 3: Water Engineering and Management Department, University of Twente, the

10 Netherlands

\section{Abstract}

12 Secondary circulation in river confluences results in a spatial and temporal variation of

13 fluid motion and a relatively high level of morphodynamic change. Acoustic Doppler

14 current profiler (aDcp) vessel-mounted flow measurements are now commonly used to

15 quantify such circulation in shallow water fluvial environments. It is well established that

16 such quantification using vessel-mounted aDcps requires repeated survey of the same

17 cross-section. However, less attention has been given to how to process these data. Most

18 aDcp data processing techniques make the assumption of homogeneity between the

19 measured radial components of velocity. As acoustic beams diverge with distance from

20 the aDcp probe, the volume of the flow that must be assumed to be homogeneous

21 between the beams increases. In the presence of secondary circulation cells, and where 22 there are strong rates of shear in the flow, the homogeneity assumption may not apply, 
23 especially deeper in the water column and close to the bed. To reduce dependence on 24 this assumption, we apply a newly-established method to aDcp data obtained for two 25 medium-sized ( $60-80 \mathrm{~m}$ wide) gravel-bed river confluences and compare the results with 26 those from more conventional data processing approaches. The comparsion confirms that 27 in the presence of strong shear our method produces different results to more 28 conventional approaches. In the absence of a third set of fully independent data, we 29 cannot demonstrate conclusively which method is best, but our method involves less 30 averaging and so in the presence of strong shear is likely to be more reliable. We conclude 31 that it is wise to apply both our method and more conventional methods to identify where 32 data analysis might be impacted upon by strong shear and where inferences of secondary 33 circulation may need to be made more cautiously.

\section{Keywords}

37 Acoustic Doppler current profiler

38 Secondary circulation

39 River confluences

40 River junctions

\section{Introduction}

42 Acoustic Doppler current profilers (aDcps) are now used widely to measure river flow in 43 three-dimensions, notably for the quantification of secondary flows. Applications have 
44 been made to river bedforms (e.g., Parsons et al., 2005; Kostaschuk et al., 2009; Shugar 45 et al., 2010), bends (e.g., Dinehart and Burau, 2005; Kasvi et al., 2013; Vermeulen et al., 46 2014a, 2015; Engel and Rhoads, 2016; Knox and Latrubesse, 2016; Kasvi et al., 2017; 47 Lotsari et al., 2017; Parsapour-Moghaddam and Rennie, 2018), junctions (e.g., Parsons 48 et al., 2007; Lane et al., 2008; Szupiany et al., 2009; Riley and Rhoads, 2012; Riley et al., 49 2015; Gualtieri et al., 2017), bifurcations (e.g., Parsons et al., 2007; Szupiany et al., 50 2012), canyons (e.g., Alvarez et al., 2017; Tomas et al., 2018; Venditti et al., 2014), deltas 51 (e.g., Czuba et al., 2011) and gravity currents (e.g., Garcia et al., 2007; Garcia et al., 52 2012). Research has also shown the need to make repeat section measurements (e.g., 53 Szupiany et al., 2007; Jackson et al., 2008) and also to process these data carefully, 54 (Muste et al., 2004; Rennie and Church 2010; Tsubaki et al., 2012; Parsons et al., 2013; 55 Petrie et al., 2013). Such processing must take into account positioning (Rennie and 56 Rainville, 2006) and orientation (Zhao et al., 2014) errors, and the treatment of repeat 57 section measurements (e.g., Szupiany et al., 2007; Jackson et al., 2008).

58 This paper is concerned with recent observations regarding the inference of secondary 59 flows from aDcp data and concerns regarding the assumption that flow is homogenous in 60 the fluid volumes defined by the acoustic beams emitted from an aDcp and used to 61 calculate any one point estimate (Vermeulen et al., 2014b). Acoustic beams are reflected 62 by suspended particles, which, if moving, cause a Doppler shift in beam frequency, which 63 is then detected at the sensor. This shift is directional so each beam measures the radial 64 velocity, which is the velocity of particle motion parallel to the acoustic path. This can be 65 assumed to be the flow velocity if the particle motion is identical to fluid motion. In order 66 to resolve flow in more than one direction, aDcps require at least three acoustic beams to 
67 estimate three Cartesian components of velocity. The radial velocities originating from the 68 beams are traditionally analyzed for a single measurement cycle at a single depth at a 69 time (Vermeulen et al., 2014b). The velocity then applies to the volume of fluid defined by 70 the beams at each depth. Flow within this volume is assumed to be homogeneous.

71 However, as the beams spread from the sensor, depth bins increase in horizontal size

72 (Rennie et al., 2002). This means that: (1) bins further from the sensor are likely to produce

73 less reliable velocities because the bin size is greater and the flow within bins is more 74 likely to be heterogeneous (Gunawan et al., 2011); and (2), even in smaller bins, velocities 75 may be less reliable in zones of strong shear where also the within-bin flow is less likely 76 to be homogeneous. In a river where measurements are made throughout the flow depth, 77 the maximum shear may be close to the bed, where the beam divergence may also be 78 greatest.

79 One solution to this problem accounts for first order shear within the flow volume (e.g. 80 Marsden and Ingram, 2004) through a Taylor expansion of the coordinate transform used 81 to determine the Cartesian velocity components. Under this solution, flow is allowed to 82 vary linearly within the bin, but the bin's volume becomes potentially larger with distance 83 from the sensor. Vermeulen et al. (2014b) developed and tested a second solution. As 84 explained in detail below, multiple radial (beam) velocity measurements within a single bin 85 are put through a Cartesian transform to obtain a localized within-bin three-dimensional 86 velocity. This method strongly reduces the volume over which homogeneity should be 87 assumed and Vermeulen et al. (2014b) found that this significantly impacted 88 interpretations of secondary velocities in the presence of strong shear. In this paper, we 89 seek to quantify the effects of this method for the measurement of secondary flow in two 
90 medium-sized river junctions (c. $60-80 \mathrm{~m}$ post-junction channel width). River junctions are 91 associated with very strong shear (e.g. Best and Roy, 1991; Biron et al., 1993, 1996a, 92 1996b; Sukhodolov and Rhoads, 2001; Rhoads and Sukhodolov, 2004, 2008; Konsoer 93 and Rhoads, 2014; Sukhodolov et al., 2017), as well as well-developed secondary 94 circulation (e.g. Ashmore et al., 1992; Rhoads and Kenworthy, 1995, 1998; Rhoads and 95 Sukhodolov et al., 2001; Lane et al., 2008; Riley and Rhoads, 2012; Riley et al., 2015). 96 Thus, understanding how to process effectively the aDcp data used to describe them is of 97 paramount importance.

\section{Methods for estimating Cartesian velocity components from aDcp data}

99 In this section, we describe the two different methodological approaches used in this study 100 to estimate Cartesian velocity components: (1) Method A, the Vermeulen et al., (2014b) 101 method; and (2) Method B, the conventional method. Common to all methods is the 102 assumption that data are available from repeat measurement of the same cross-section, 103 as has been shown to be critical for obtaining reliable estimates of secondary circulation 104 from aDcp data (Szupiany et al., 2007), particularly when single transect measurements 105 are not close enough together.

\section{Method A: based on Vermeulen et al., (2014b)}

107 Application of the Vermeulen et al. (2014b) method requires mapping of radial beam 108 velocity data onto a predefined mesh. This mesh requires both a bottom topography or 109 bathymetric model, and an upper limit just below the water surface. As the measurements 110 were made using several repeat transects for each cross section, the first step is to define 111 a mean cross section for each set of individual transects (boat tracks). The second step 
112 is to define a grid mesh for this mean cross section. Third, all measured beam velocities

113 are projected on to this cross section mesh. Finally, the beam velocities within each mesh

114 cell are then used to resolve a Cartesian velocity for the mesh cell. Errors that influence

115 these steps can be estimated.

116 The first step is estimation of the mesh extremes, both the lower boundary or bathymetry

117 model and the upper boundary near the water surface. To generate the bathymetry model

118 we use depth soundings collected with the aDcp. We recognize that each beam may

119 register a different distance of the stream bed from the sounder, especially as we are

120 dealing with bathymetrically irregular cross-sections. Specifically, for each bottom track

121 sounding within each transect, we use the UTM coordinates obtained with a coupled

122 differential GPS (dGPS), the range of each bottom track beam return, and the instrument

123 tilt to estimate the bed elevation and horizontal position of each beam impingement point

124 on the bed. These bed positions are combined together to identify an initial mean transect.

125 Provided a point is within a certain distance from the initial mean cross-section, LOWESS

126 interpolation (Appendix A) is applied, which has the effect of defining a bathymetric model

127 that gives most weight to points that appear to be closer to the cross-section. It is important

128 to note that this mean transect is not necessarily orthogonal to the primary flow direction

129 and so will not yield true primary and secondary flow estimates without further correction.

130 We address this below.

131 Once the initial bathymetric model is defined, we estimate a unique vector using the initial

132 mean transect; that is the principal direction of the scatter cloud of all $x$ and $y$ UTM 133 positions at the bed. This unique vector points in the direction of the largest eigenvector 134 of the covariance matrix of all UTM positions $(t)$. We then calculate the mean UTM position 
$135\left(p_{\text {mean }}\right)$ for each set of individual transects and the difference between each measured 136 beam position $\left(p_{b}\right)$ and the mean position. The dot product of these obtained values and

137 the unique vector is then used to define the projection of each UTM position in the direction

138 of the unique vector. To identify the final mean cross section, we sum up all individual

139 projected vectors and obtain the best fit to all available data (Figure 1).

140 To define the upper boundary of the mesh, we estimate the elevation of the water surface.

141 As there is a blanking distance at the surface of the water during the measurement, we

142 then remove this blanking distance, taken as $0.30 \mathrm{~m}$. Thus, the mesh has also a blanking

143 distance and the upper part of the cross-section is, strictly, the upper limit of available 144 data, not the water surface.

145 "Figure1"

146 The second step uses the defined bathymetric model and available velocity bins within 147 the measured area (not influenced by side lobes, and below the blanking distance) to 148 define a cross-section mesh. The side-lobe interference is caused by the striking of the 149 channel bed by side-lobe energy from each of the acoustic beams. This side-lobe energy 150 has strong reflections from the bed, which result in echoes that overwhelm the signal from 151 scatters near the bed. The thickness of the side-lobe layer is typically $6-7 \%$ of the 152 measured depth (Morlock, 1996).

153 To generate the mesh, the cross section is initially subdivided into vertical slices with equal 154 widths $(\Delta \mathrm{n})$. For each slice, the simplest definition of mesh cell thicknesses $(\Delta \mathrm{z})$ divides 155 each vertical equally. These verticals are converted to non-dimensional $\sigma$ coordinates 156 using following equation: 
$157 \quad \sigma=1-\left(\frac{p_{v} \cdot \boldsymbol{k}-\eta}{p_{b} \cdot \boldsymbol{k}-\eta}\right) \quad$ (Vermeulen et al. 2014b)

158 where $p_{v}$ stands for velocity measurement positions $(\mathrm{m}), p_{b}$ is the corresponding bed 159 position $(\mathrm{m})$ that is found using velocity measurement horizontal positions and applying 160 the bathymetric model, $\boldsymbol{k}$ is the upward pointing unit vector and $\eta$ are the water surface 161 fluctuations around the mean water level at which $\mathrm{z}=0$.

162 However, because of beam spreading and differences in the distance of the sounder from 163 the bed, which varies with position of the sounder, this tends to produce a highly 164 heterogeneous number of measurements in each cell within the mesh. The alternative, 165 adopted here, is to allow mesh cell thickness to vary through the water column such that 166 there is a roughly equal number of beam velocities contributing to each mesh cell (see 167 Figure 2 for a typical distribution).

168 As the river bed form is varying, to follow its shape, each mesh cell is considered to be a 169 cuboid with 6 edges, two on the left side, two in the middle and two on the right side. To 170 define these edges, the first step is to define the middle point of each mesh cell. Once 171 defined, by calculating the slope for each half part of the mesh cell, edges can be obtained.

172 The mesh cell faces are then calculated on the basis of adjacent verticals and the mesh 173 cell upper and lower boundaries.

174 To identify the beams that contribute to each mesh cell, an index for each beam velocity 175 is defined, which shows its associated mesh cell, using the projection of each radial 176 velocity onto the estimated mean cross section (Figure 2).

177 "Figure 2" 
178 In the third step, the radial velocities for each beam (b) that contribute to each mesh cell 179 (the $\mathrm{N}$ beam velocities) have to be transformed into Cartesian velocities $\left(\mathrm{v}_{\mathrm{x}}, \mathrm{v}_{\mathrm{y}}\right.$ and $\left.\mathrm{v}_{\mathrm{z}}\right)$ 180 using:

$181 \quad\left(\begin{array}{c}b_{1} \\ \vdots \\ b_{N}\end{array}\right)=\left(\begin{array}{c}q_{1} \\ \vdots \\ q_{N}\end{array}\right) \cdot\left(\begin{array}{c}v_{x} \\ v_{y} \\ v_{z}\end{array}\right) \leftrightarrow b=Q \cdot u$

182 where $\mathbf{q}$ is a unit vector which describes the direction of the acoustic beam.

183 To obtain the raw beam velocities, we use matrix transformations obtained from the raw 184 data to transform measured velocities in $X Y Z$ coordinates into beam velocities. The 185 Vermeulen et al., (2014b) method includes in the transformations an explicit treatment of 186 the random errors due to internal and external factors and the bias (systematic errors) 187 caused by the measurement system and the nature of river flow (Tsubaki et al., 2012). 188 Random errors include those that come from sampling a time-varying flow in the presence 189 of strong gradients and represent a form of aliasing. By adding a combined term of errors $190 \varepsilon,(2)$ becomes:

$191 \quad b=Q u+\varepsilon$

192 A least squares solution is fitted to (3) that minimizes the sum of the square of the errors.

193 The optimal estimation $(\widehat{\mathbf{u}})$ for $(\mathbf{u})$ is then given by the normal equation:

$194 \widehat{\mathbf{u}}=\mathrm{Q}^{+} \mathbf{b}+\varepsilon$

195 where $Q^{+}$can be defined as:

$196 Q^{+}=\left(Q^{\top} Q\right)^{-1} Q^{\top}$ 
197 To solve three Cartesian velocity components, we need at least three equations. Each 198 beam measurement in a mesh cell adds an equation. Where enough beam velocities are 199 collected in a mesh cell and the equations are different from each other (beam velocities 200 are measured from different directions), the velocity can be estimated. To check whether 201 this is the case, the matrix describing the system of equations can be analyzed. In the 202 processing we use the rank which indicates how many unknowns can be solved from the 203 system of equations. When the rank is three, the three Cartesian velocities can be solved. 204 Where the rank of the matrix is one or two, the system cannot be solved. Where the 205 system of equations is overdetermined, the obtained solution is a matrix with more 206 equations (rows) than unknowns (columns). The velocity can be solved using the 207 generalized inverse of the matrix and in such a way that the sum of squared errors is 208 minimized. As this combined term of errors also contains information about the turbulence 209 and accuracy of the measurements, we can obtain the covariance matrix of the velocity 210 components:

$211 \hat{\varepsilon}=\mathbf{b}-Q \widehat{u}$

$212 \operatorname{var}(\widehat{\mathbf{u}})=\frac{\hat{\varepsilon}^{\top} \hat{\varepsilon}\left(Q^{\top} Q\right)^{-1}}{\mathrm{~N}-3}$

213 and the variance of the velocity across the section can be then estimated as:

$214 \operatorname{var}(\mathbf{u})=\frac{\operatorname{var}(\hat{\mathbf{u}})}{\mathrm{N}}$

\section{Method B: the standard aDcp method}

216 As the Doppler shift is directional, it can only measure radial velocities. With the standard 217 method, to determine Cartesian velocity components, radial velocities then have to be 
218 resolved into three orthogonal velocity vectors. To do so, at least three beam velocities 219 pointed in known directions are required. Also, because the beams are measuring 220 different water profiles along their individual slant ranges, the assumption of horizontal 221 homogeneity must be taken into account. Hence, in the standard method, the three 222 dimensional velocity for each depth bin for each ping can be solved for a typical four-beam 223 system using the following equations (Mueller and Wagner, 2009):

$224 \quad V_{x}=\frac{\left(b_{3}-b_{1}\right)}{\sqrt{2} \sin \theta}$

$225 \quad V_{y}=\frac{\left(b_{4}-b_{2}\right)}{\sqrt{2} \sin \theta}$

$226 \quad V_{z}=\frac{-\left(b_{1}+b_{3}\right)}{(2 \cos \theta)}=\frac{-\left(b_{2}+b_{4}\right)}{(2 \cos \theta)}$

227 where $V_{y}$ is the cross stream velocity assuming beam 3 is pointed upstream, $V_{x}$ is the 228 streamwise velocity, $V_{z}$ is the vertical velocity, $b_{1}, b_{2}, b_{3}$ and $b_{4}$ are the radial velocities 229 measured in beams $1,2,3$ and 4 respectively and $\theta$ is the tilt angle of the beams referenced 230 to vertical. These data should then be corrected for pitch and roll angles, obtained from 231 the internal inclinometer and the heading angle from the internal compass. Velocity 232 outputs are already corrected for ship velocities.

233 To compare results obtained using Method B with those of Method A, we use the same 234 mean cross section built for Method A, as well as the same bathymetric model and the 235 same mesh. Each measured velocity vector is assigned to the appropriate mesh cell by 236 projecting its $3 \mathrm{D}$ position (horizontal position and depth) onto the mean cross section 237 mesh. We then average $x, y$, and $z$ components of all velocities measured within a mesh 238 cell to obtain the mean velocity vector for the mesh cell. 


\section{Methodology}

240 This paper is motivated by the need to acquire three-dimensional data from junctions of

241 tributaries with a main river stem, here the River Rhône, western Switzerland, and so the

242 need to identify methods for reliably obtaining Cartesian velocities from aDcp data. The

243 Rhône tributaries typically have very high bedload transport rates for short periods of time,

244 leading to the formation of very large tributary mouth bars downstream of their junctions

245 with the main river. These bars are maintained for weeks or months such that at lower

246 tributary flow, with negligible sediment supply, there is a legacy effect of previous high

247 momentum tributary events upon junction morphology and secondary flow formation.

248 For this paper, we used a specially-designed rope and pulley system to collect aDcp data 249 from the junction of two tributaries with the Rhône (Figure 3).

250 "Figure 3"

251 The Lizerne is a Rhône tributary of almost $20 \mathrm{~km}$ length that flows south-westward from 252 the western slopes of the Tête Noire $(2451 \mathrm{~m})$ or La Fava $(2612 \mathrm{~m})$, in the Bernese Alps.

253 This river is heavily regulated for hydropower with sediment extracted upstream of the 254 junction. As a result, there is negligible sediment supply and no evidence of point bar 255 formation. It reaches the Rhône between Ardon and Vétroz, forming a $90^{\circ}$ junction angle 256 and it has a bed that is nearly concordant with the Rhône.

257 The Grande Eau is a second tributary of the Rhône River which has a length of $26 \mathrm{~km}$ and 258 takes its source on the Vaud side of the Les Diablerets and flows into the Rhône River 259 with a $70^{\circ}$ confluence angle, near Aigle. The Grande Eau bed is $1.5 \mathrm{~m}$ higher than the 260 Rhône such that it is markedly discordant. 
261 In this section, we: (1) describe the aDcp used to collect data; (2) describe how the aDcp 262 was deployed; and (3) outline the analytical approaches used to interpret the results from 263 the different methods. Although the method is valid for any aDcp that has an onboard 264 compass and potential for differential GPS positioning, as is standard with most aDcps, 265 we use a Sontek M9 aDcp in this study.

\section{The Sontek M9 aDcp}

267 The SonTek M9 aDcp is a nine-transducer system with three acoustic frequencies, 268 configured as two sets of four profiling beams $(3 \mathrm{MHz}$ and $1 \mathrm{MHz}$ transducers in Janus 269 configurations) and one vertical beam (0.5 MHz Echo sounder) for depth measurements 270 (SonTek YSI, 2010). It uses these two sets of four beams to provide raw radial velocity 271 samples. These beams are equally spaced at $90^{\circ}$ azimuth angles and are projected at an 272 angle $\theta$ of $25^{\circ}$ off the vertical axis (SonTek YSI, 2000). For the standard configuration, 273 the four beams encompass a sampling diameter of $93 \%$ of the distance from the aDcp 274 (7\% of side-lobe) (SonTek YSI, 2000).

275 The output velocities from the SonTek M9 Riversurveyor are either in Cartesian 276 coordinates $(\mathrm{XYZ})$ that are relative to sensor orientation or in Earth coordinates (ENU) for 277 a SonTek system with compass and tilt sensors. These raw velocity data in Earth 278 coordinates or XYZ coordinates are already corrected for the ship motion. To apply 279 Method A to Sontek output data, as this method is based on radial velocities, it is 280 necessary to transform these output velocities to radial velocities. To do so, we add ship 281 velocities to these output velocities and then apply the inverses of the instrument's matrix 282 coordinate transformations (obtained from MATLAB files output by the SonTek data 283 collection software RiverSurveyor). As the survey is being undertaken using a moving 
284 vessel, these radial velocities then have to be corrected again for the boat velocity. There 285 are two key methods for doing this. The first uses the bottom tracking to measure the boat 286 velocity relative to the river bed, under the assumption that the latter is stationary (i.e. 287 there is no bedload transport). The second tracks the boat position using differential GPS 288 (dGPS, e.g. Zhao et al., 2014). In this study, we corrected all raw beam velocities for ship 289 velocities, using dGPS as we could not exclude the possibility of there being bedload 290 transport.

291 To apply Method B in this study, we use the raw velocity data in Earth coordinates and 292 we correct it for pitch and roll angles, obtained from internal inclinometer and heading 293 angle data for the internal compass. For SonTek M9 aDcps, pitch is a y-axis rotation and 294 roll an $\mathrm{x}$-axis rotation.

295 Depending on the water depth and velocity, the Sontek M9 firmware changes the acoustic 296 operating frequency and the water profiling mode on-the-fly, thus the number of sampled 297 points in the vertical varies automatically from one profile to the next. Specifically, when 298 the water is shallower than $0.75 \mathrm{~m}$ and the maximum velocity is less than $0.4 \mathrm{~ms}^{-1}$, the 299 M9 reports data acquired with a $3 \mathrm{MHz}$ frequency using the pulse coherent mode to obtain 300 a 2cm depth measurement resolution. For deeper situations, this frequency changes to 1 $301 \mathrm{MHz}$ pulse coherent pings using a $6 \mathrm{~cm}$ aDcp cell size. If the maximum velocity is greater 302 than $0.4 \mathrm{~ms}^{-1}$ then SmartPulse (i.e., broadband) mode is utilized, with the $3 \mathrm{MHz}$ beams 303 if depth is less than $5 \mathrm{~m}$ and the $1 \mathrm{MHz}$ beams if depth is greater than $5 \mathrm{~m}$, with the aDcp 304 cell size optimized based on the current water depth. As a result of these on-the-fly 305 changes, each measured profile has a different number of aDcp cells and different aDcp 306 cell sizes. Hence, to correct the aDcp cell size variability, for both methods A and B there 307 is the need to define a cross-sectional mesh and to project the measured velocities to this 
308 mesh. For Method A we use the beam velocity vertical positions in a non-dimensionalized 309 coordinate system using equation 1, within the predefined mesh explained in section 2.1.

\section{Deployment of the Sontek M9 in the river junctions}

311 The survey work was undertaken in two junctions of the Swiss River Rhône, the Lizerne312 Rhône confluence in August 2017 and the Grande Eau-Rhône confluence in May 2018, 313 using a Sontek M9 vessel mounted aDcp and a specially-designed rope-pulley system 314 (Figure 3c). The survey was spatial, monitoring 11 cross-sections from upstream of the 315 junction to its downstream at the Lizerne-Rhône confluence with a Momentum ratio (Mr) 316 of 0.018 (Figure 3a) and 11 cross-sections at the Grande Eau-Rhône confluence with a 317 Mr of 0.022 (Figure 3b). Table 1 shows the general characteristics of these two 318 confluences on the date of the measurements.

319 "Table 1"

320 As proposed previously by Dinehart and Burau (2005), Szupiany et al. (2007), Gunawan 321 et al. (2011) and Vermeulen et al., (2014b) at least five repeats are required to have a 322 robust estimation of secondary velocities. Hence, in this paper, data are processed for 323 cross-section 6 at the Lizerne-Rhône confluence (Figure 3a), and for cross section 3 at 324 the Grande Eau-Rhône confluence (Figure 3b). Identification of the minimum number of 325 repeat transects necessary per cross-section was undertaken using cross-section 9 at the 326 Lizerne-Rhône confluence (Figure 3a), which involves 16 repetitions. We noted that after 327 application of Method A, the standard deviation of velocity stabilized with six repetitions, 328 which is the number we adopt for this study. 


\section{Bin position error determination}

330 Application of Method A requires estimation of the error terms in (2). The size of the 331 sampling volume in each beam is determined by the size of the bin used. As the SonTek

332 M9 aDcp uses different bin sizes depending on the water track frequency (section 2.1.3), 333 these volumes could vary. Applying Method A might improve the velocity estimation for 334 large measurement volumes at depth, as it does not rely on the homogeneity assumption. 335 But as bins with a small number of velocity measurements will have greater error, this 336 method can estimate velocities with error. Also, if the beam velocity distribution within 337 each mesh cell is not linear, as averaging is made in the middle of each mesh cell, it can 338 introduce error in velocity estimation. Thus, it is necessary to calculate a minimum 339 necessary mesh cell size when applying Method A.

340 Method B is inherently limited by spatial averaging due to the potential use of divergent

341 beams and the associated homogeneity assumption. In other words, one must assume 342 that the velocity is homogeneous over the horizontal domain defined by beam divergence 343 (Eq.12). Method $\mathrm{A}$ has the advantage that velocities are recorded within an individual 344 beam depth bin, thus no spatial averaging between beams is required. However, in order 345 for Method A to overcome the uncertainty induced by spatial averaging inherent to Method $346 \mathrm{~B}$, it is essential that the bin location is known explicitly. Error in bin location can be induced

347 by dGPS position and or tilt sensor (pitch and roll) errors. We therefore compare possible 348 bin position errors using Method A to beam divergence obtained from Method B to indicate 349 when Method A should be advantageous over Method B.

350 Beam divergence is the spatial separation of the beams due to the Janus configuration of 351 the beams with beam angles of $25^{\circ}$. This divergence determines the sampling volume that 
352 must be considered homogeneous for Method B and can be calculated using equation 353 12:

$354 x_{b}=2 d \tan \theta$

355 where $d$ is the depth in $m$ and $\theta$ is the beam angle which for a SonTek aDcp is $25^{\circ}$. The

356 aDcp dGPS is used to reference the velocity measurements in space and to estimate the 357 ship velocity. If dGPS is used for ship velocity, this introduces errors in measurement of 358 the absolute water velocity (because ship velocity is subtracted from the water velocity 359 measured in the reference frame of the aDcp). This uncertainty introduces error in velocity 360 calculations.

361 To estimate the errors due to dGPS and the tilt sensors, in this study we assume normally 362 distributed random errors with a standard deviation of $\pm 1^{\circ}$ for tilt sensors, based on 363 manufacturer specifications, and a normally distributed displacement error measured by 364 the dGPS for the dGPS positions (as a function of satellite configuration during 365 measurement), and we apply a Monte Carlo approach which we run 100 times sampling 366 under these uncertainties. Each time we calculate the estimated secondary velocity 367 differences as compared with the original secondary velocities.

368 To be able to reduce the uncertainty due to velocity estimation using Method A compared 369 to Method B, the errors induced in Method A related to GPS uncertainty and tilt sensors 370 must be less than the errors in Method B due to beam divergence and the homogeneity 371 assumption. Hence, Method A can be used if the error associated with a minimum aDcp 372 cell size is in between the error due to beam divergence and the maximum estimated error 373 due to the GPS and tilt sensors. Otherwise using this method introduces more error in 374 velocity estimations than using Method B. 


\section{Data interpretation}

376 Methods A and B, described above, were applied to the Sontek M9 data, to determine 377 Cartesian velocities $\left(v_{x}, v_{y}\right.$ and $\left.v_{z}\right)$. As our interest is in process estimation, here we 378 describe the methods we apply to the Cartesian velocities to estimate processes relevant 379 to junction dynamics. In order to distinguish between primary and secondary components 380 of flow, we need to rotate the initial mean transect. Options for doing this are reviewed in 381 Lane et al. (2000) and we do not assess them here, but rather apply the zero net cross 382 stream discharge definition (Lane et al., 2000). By calculating the mean values of the $x$ 383 and $y$ velocity components $(U$ and $V$ ), we then calculate the velocity magnitude $(v)$. By 384 rotating these velocity components to the direction of the cross-stream velocity, using the 385 unique vector $(\boldsymbol{\sigma})$, primary velocity vectors $\left(\boldsymbol{v}_{\boldsymbol{p}}\right)$ and secondary velocity vectors $\left(\boldsymbol{v}_{\boldsymbol{s}}\right)$ then 386 can be estimated.

$387 v=\sqrt{U^{2}+V^{2}}$

$388\left(\begin{array}{l}\sigma_{x} \\ \sigma_{y}\end{array}\right)=\left(\begin{array}{l}U \\ V\end{array}\right) / v$

389 where $\sigma_{x}$ and $\sigma_{y}$ are sin and cos of the angle between the section angle and east.

$390 \quad \boldsymbol{v}_{p}=\sigma_{x} \boldsymbol{v}_{x}+\sigma_{y} \boldsymbol{v}_{y}$

$391 \quad \boldsymbol{v}_{s}=-\sigma_{y} \boldsymbol{v}_{x}+\sigma_{x} \boldsymbol{v}_{y}$

392 However, secondary circulation is all flow that is orthogonal to the primary flow and not 393 just horizontal flow; there should be not net secondary flux in a section; and so correction 394 should also consider vertical velocities. Thus, we extend these relationships to include 395 vertical velocities: 
$396 \quad\left(\begin{array}{lll}\sigma_{x, 1} & \sigma_{x, 2} & \sigma_{x, 3} \\ \sigma_{y, 1} & \sigma_{y, 2} & \sigma_{y, 3} \\ \sigma_{z, 1} & \sigma_{z, 2} & \sigma_{z, 3}\end{array}\right)=\left(\begin{array}{c}U \\ V \\ W\end{array}\right) / v$

397 where: $U, V$ and $W$ are the mean velocities of $x, y$ and $z$ velocity components, respectively 398 and $v$ is the magnitude of the velocity which can be obtained using:

$399 v=\sqrt{U^{2}+V^{2}+W^{2}}$

$400 \quad \boldsymbol{v}_{p}=\sigma_{x, 1} \boldsymbol{v}_{x}+\sigma_{x, 2} \boldsymbol{v}_{y}+\sigma_{x, 3} \boldsymbol{v}_{z}$

$401 \quad \boldsymbol{v}_{s}=\sigma_{y, 1} \boldsymbol{v}_{x}+\sigma_{y, 2} \boldsymbol{v}_{y}+\sigma_{y, 3} \boldsymbol{v}_{z}$

$402 \quad \boldsymbol{v}_{v}=\sigma_{z, 1} \boldsymbol{v}_{x}+\sigma_{z, 2} \boldsymbol{v}_{y}+\sigma_{z, 3} \boldsymbol{v}_{z}$

403 To estimate velocity gradients, and to correct for weak curvature with the survey method 404 at the edges of each transect line (e.g. Figure 3), all data have been transformed into row 405 and column coordinates ( $\eta$ and $\zeta$ ) using the following transformation:

$406 \quad\left(\begin{array}{c}\frac{\partial}{\partial n} \\ \frac{\partial}{\partial z}\end{array}\right)=\left(\begin{array}{ll}\frac{\partial \eta}{\partial n} & \frac{\partial \zeta}{\partial n} \\ \frac{\partial \eta}{\partial z} & \frac{\partial \zeta}{\partial z}\end{array}\right)\left(\begin{array}{c}\frac{\partial}{\partial \eta} \\ \frac{\partial}{\partial \zeta}\end{array}\right)$

407 where $n$ and $z$ are horizontal and vertical coordinates on the section plane, respectively 408 (Vermeulen et al., 2014b).

\section{Results}

410 Primary and secondary velocities

411 Primary and secondary velocities estimated using methods $A$ and $B$ for the Lizerne-Rhône 412 confluence appear to be similar at cross-section 6 (Figures $4 a$ and $4 b$ ) and the differences 
413 in estimated secondary flows are minor. The differences are most pronounced between 41410 and $5 \mathrm{~m}$, in the middle of the main channel.

415 These primary and secondary velocity patterns show higher differences at cross-section 4163 of the confluence of Grande Eau-Rhône (Figures $4 c$ and $4 d$ ) despite it having a similar 417 momentum ratio to the Lizerne during measurement. Primary velocities differ significantly 418 between methods A and B: (1) at greater distance from the aDcp because the bins contain 419 larger volumes of water assumed to be homogenous; and (2) at the edges of the cross420 section where there are more beam velocity measurements (contours in Figures $4 \mathrm{c}$ and $4214 d$ ). Secondary velocity vectors estimated using Method A indicate flow convergence at 422 the surface and flow descending towards the riverbed throughout the centre of the channel 423 (Figure 4c). This is due to a high degree of bed discordance between the Grande Eau and 424 the Rhône, which increases the penetration of the tributary flow into the main channel over 425 the junction, and which forms a zone of high lateral and vertical shear, on the one hand, 426 and main channel narrowing because of penetration of the tributary mouth bar on the other 427 hand. The secondary velocity vectors estimated by Method B show a weaker penetration 428 of the tributary flow into the main channel, which results in a reverse flow towards the bank 429 on the tributary side of the channel at the surface of the mixing interface (Figure $4 d$ ). In 430 this case, the core of the secondary circulation is located in the middle of the main channel 431 and closer to the inner bank.

432 "Figure 4"

433 Figure 5 and Figure 6 quantify the differences in primary and secondary velocity patterns 434 estimated using methods A and B, for the Lizerne-Rhône confluence. Figures $5 \mathrm{a}$ and $5 \mathrm{c}$ 435 and Figures $6 a$ show that almost $4 \%$ of mesh cells have a relative difference in primary 
436 velocities between methods $A$ and $B$ of more than $10 \%$. These differences can exceed $437 \quad 0.2 \mathrm{~ms}^{-1}$ and so they are relatively small. Velocity differences are more pronounced in 438 estimated secondary velocities, with almost $82 \%$ of mesh cells having a difference of more 439 than $10 \%$, and almost $37 \%$ of mesh cells having a difference of more than $50 \%$ (Figure $4405 b, 5 d$ and $6 b)$.

441 "Figure 5"

442 "Figure 6"

443 At the Grande Eau-Rhône confluence, these differences are greater as compared with 444 those of the Lizerne-Rhône confluence. Figures 7a, 7c and 8a show that these differences 445 for primary velocities exceed $0.4 \mathrm{~ms}^{-1}$ in the zone of high vertical and lateral shear and 446 near the inner bank. Almost $20 \%$ of the mesh cells have a relative difference in primary 447 velocities between methods $A$ and $B$ of more than $10 \%$. The secondary velocity 448 differences are more pronounced between these two methods. Figures $7 b$ and $7 d$ show 449 differences with a magnitude of $0.4 \mathrm{~ms}^{-1}$ near the edges and near the bed. Almost all the 450 mesh cells have a difference in estimated secondary velocities between two methods.

451 Figure $8 \mathrm{~b}$ shows that almost $93 \%$ of the mesh cells have a relative difference of $10 \%$ 452 between methods $A$ and $B$. although this value decreases to $55 \%$ for a relative difference 453 of $90 \%$ between these two methods.

454 "Figure 7"

455 "Figure 8" 


\section{Velocity gradients}

457 As Figure 9 shows, there is a strong relationship between lateral gradient in secondary 458 velocities and differences between the secondary velocities estimated using methods $A$ 459 and B for both the Lizerne-Rhône and the Grande Eau-Rhône confluences. This is 460 because a stronger velocity gradient increases the probability that the assumption of flow

461 homogeneity within a bin is likely to fail. Indeed, the marked differences between methods $462 \mathrm{~A}$ and B at the Grande Eau confluence (Figure 7) are also in a zone of strong lateral shear.

463 "Figure 9"

\section{$464 \quad$ Number of repeat transects}

465 One way to reduce data fluctuations due to random errors and turbulence, during the 466 measurement using moving vessel aDcps, is to average by using several repeat transects 467 together in one cross section. As each estimated velocity measurement is a single sample 468 in time, adding in a repeat section adds in an additional estimated velocity measurement. 469 Under [8], this should cause the variance to increase, despite the number of 470 measurements used in its estimation increasing, until the point at which there are enough 471 repeats to capture the effects the range of scales of variation in turbulence impacting the 472 measurement. Then, this variance will become stable. At this stage we can consider the 473 number of repeats as the minimum number required to have a robust estimation of 474 secondary velocity vectors that is to have reached estimates of velocity that are 475 asymptotic on this stable state.

476 Here we apply both methods A and B to the survey of 16 repeats at cross-section 9 in 477 Figure $3 a$ at the Lizerne-Rhône confluence. To allow a reasonable comparison, three 478 mesh cells in the middle of the cross section, and at three different depths (near the 
479 surface, middle depth and near the bed) have been chosen (Figure 11). Results show 480 that by using Method $A$, after six repeats, a stable variance of the velocity estimator is 481 obtained at the Lizerne-Rhône confluence (Figure 11a). Many more repeats are needed 482 using Method B (Figure 11b) and this is likely because Method B uses fewer 483 measurements per mesh cell. These results also show a higher standard deviation of the 484 velocity estimation near the surface, using Method A and before achieving the stable 485 situation. This can be explained by the fact that near the surface Method A is more 486 sensitive to errors caused by positioning, while near the bed, hence with distance from 487 the sounder, as the beam spread increases, the improvement obtained using Method $A$ 488 is more pronounced (Figure 11a).

489 "Figure 10"

490 "Figure 11"

491 DGPS and tilt sensor uncertainty analysis

492 As explained above a normally distributed random error has been applied 100 times to 493 both dGPS positioning (by adding a random offset) and tilt sensors (by changing pitch and 494 roll angles randomly) and the secondary velocities have been estimated using Method $A$ 495 for each perturbed dataset. As Figure 12 shows, the magnitude of errors related to dGPS 496 accuracy are higher than those related to tilt sensor accuracy, for both confluences. These 497 values can reach $\pm 0.03 \mathrm{~ms}^{-1}$ and confirms the earlier finding of Rennie and Rainville 498 (2006) which showed that GPS corrections can have average errors of about $\pm 0.03 \mathrm{~ms}^{-1}$ 499 (Figures 12a and 12c). These magnitudes are also higher near the surface and near the 500 bed for the Lizerne-Rhône confluence (Figure 12a). Near the surface, as there fewer 501 measurements that can contribute to the estimation of aDcp position and tilt, uncertainties 
502 in dGPS data will have a greater effect. Near the bed, as the velocity gradient is higher, 503 errors will be greater as well. Figure 12c shows higher magnitudes near the surface at 504 cross-section 3 in Figure 3b for the Grande Eau-Rhône confluence.

505 Errors related to tilt sensor uncertainty are higher where there is a higher velocity gradient. 506 This is related to the fact that within the mesh cells with higher velocity gradients, as the 507 velocity distribution is not linear, and as averaging is made in the middle of the mesh cell, 508 it is more probable that the velocity will be affected by sensor inaccuracies of bin 509 positioning, and so be in error (Figures 12b and 12d).

510 "Figure 12"

\section{Homogeneity assumption analysis}

512 Figure13 shows the maximum inhomogeneity allowance, using Method B for both case 513 studies. These results are obtained by dividing the velocity gradient obtained from 514 equation 22 by the divergence of the beams from equation 12 . They confirm that, for the 515 homogeneity assumption to be valid and thus error to be minimized using Method B, the 516 maximum mesh cell size, which can be used is as small as $5 \mathrm{~cm}$ near the bed. Clearly, 517 this is impossible as the configuration of the beams using aDcps always results in beam 518 divergence greater than $5 \mathrm{~cm}$.

519 "Figure 13"

520 Primary and secondary flow patterns

521 In this section, we compared estimated primary and secondary velocities using methods $522 \quad A$ and $B$ for other cross sections in Figure 3 for both river confluences. 
523 Figure 14 shows the results for cross sections 4, 5 and 7 (in Figure 3a) at the Lizerne524 Rhône confluence. These cross sections also show similar results in primary and 525 secondary velocity patterns for both methods A and B. Figure 15 shows different patterns 526 in primary and secondary velocities estimation using Method A and B for cross sections 5274,6 and 8 in Figure 3b at the Grande Eau-Rhône confluence. Method A leads to the 528 identification of a stronger and more coherent tributary penetration at cross-section 4 and 529 weaker upwelling mid-channel, giving the impression of less intense secondary circulation 530 (Figure 15). At section 6, flow towards the true left across the shallow top of the tributary 531 mouth bar is identified and is coherent with Method A. At the channel-scale there is 532 general flow convergence reflecting channel narrowing (Figure 15). When using Method $533 \mathrm{~B}$, these patterns are less coherent and flow is towards the true right in the vicinity of the 534 tributary mouth bar. These patterns are repeated for section 8 (Figure 15).

535 "Figure 14"

536 "Figure 15"

\section{Discussion}

538 In this paper we used data collected with boat-mounted aDcp technology at two 539 confluences of the Swiss river Rhône, both with similar and very low momentum ratios $540(0.018,0.022)$ and analysed these using two different methods, $A$ and $B$, to estimate 541 Cartesian velocity components. Method A is based on a methodological approach 542 developed by Vermeulen et al. (2014b). It differs by treating explicitly each individual beam 543 velocity based on its position within a predefined mesh. Results show that this method 544 reduces the volume over which the flow must be assumed to be homogenous (Fig 13). It 
545 can, but not necessarily does, result in differences in estimated primary and secondary 546 velocities as compared with the more traditional method ( $B$ in this study), that involves 547 determining velocities by averaging data from the spreading beams. Our results show that 548 these differences are more pronounced in estimated secondary velocities than primary 549 velocities and are higher where there is a greater lateral velocity gradient (Figure 9). The 550 comparison between the two case studies shows that even though both confluences have 551 a very low momentum ratio, as the confluence of the Grande Eau-Rhône has a more 552 complex shear zone, likely due to the effects of bed discordance, and there are more 553 significant differences in the estimation of primary and secondary velocities. This is related 554 to the extent to which spreading of the aDcp measurement beams influences the 555 secondary velocities, particularly in relation to lateral gradients in flow conditions. More 556 standard methods (Method B in this study) are valid if the flow is completely homogenous 557 over the diameter of the fluid column that the beams spread. This diameter varies over 558 depth and is largest near the bed. In the case of the Grande Eau-Rhône confluence where 559 stronger lateral velocity gradients exist in the flow, individual beams will not be measuring 560 homogenous conditions, particularly near the bed and in the zone of high shear near the 561 inner bank, because the spread of the beams may be greater in diameter than the width 562 of the zone of lateral velocity variation. In this case, as Method A involves less spatial563 averaging than Method B, it may provide more accurate information on the flow behavior, 564 but such a conclusion really needs a third and independent method to confirm it. At the 565 Lizerne-Rhône confluence, even though the momentum ratio is similar to Grande Eau566 Rhône confluence, there is only more localized shear in the flow and a simplified shear 567 zone (Figure 9). In such a situation, using Method B to detect the large scale patterns of 568 secondary flow may be more advantageous, because it involves more spatial averaging. 
569 The above discussion suggests that whether or not high rates of later shear influence the 570 potential importance of Method A depends on distance from the aDcp: with more 571 divergence at greater depths, lower levels of lateral shear are likely to be acceptable.

572 Figures $16 \mathrm{a}$ and $16 \mathrm{~b}$ quantifies the relationship between lateral velocity gradient, depth 573 and the magnitude of the relative differences in secondary velocities estimated using 574 methods $A$ and $B$ for the cross-section 6 of the Lizerne-Rhône and cross-section 3 of the 575 Grande Eau-Rhône confluences, respectively. At the Lizerne-Rhône confluence, as the 576 zone of high lateral shear is absent, even though there is a strong relationship between 577 the magnitude of the relative differences in secondary velocities estimated using methods $578 \quad A$ and $B$ and the depth (Figure 16a), their relationship with the lateral velocity gradients is 579 poor. In contrast, for the case of the Grande Eau-Rhône confluence (Figure 16b), where 580 increasing the lateral velocity gradient and depth results in higher relative differences in 581 secondary velocities. Thus, the need to use Method A will depend on the case being used 582 and the extent to which there is lateral shear at greater distances from the aDcp. This is 583 why whilst it may be tempting to introduce some kind of shear or velocity gradient 584 threshold to identify when Method A might be preferable, to do so would be misleading as 585 the threshold will also depend on the distance of the shear from the aDcp.

586 "Figure 16"

587 Results also confirm that several repeat transects are indispensable to provide a robust 588 estimation of secondary circulation and to reduce the effect of spatial inhomogeneity and 589 temporal variations. Although Method A reduces the minimum number of repeat transects 590 needed to estimate the secondary velocities, a larger number of these minimum repeat 591 transects (6 or more repeats for Lizerne-Rhône confluence) appeared to be required. This 
592 is higher than in the earlier findings of Szupiany et al. (2007) and Vermeulen et al., (2014b) 593 who argue that 5 repeats are enough to have a robust estimation of the turbulence 594 averaged velocity. We also note that an even number of repeats may be important to 595 avoid directional bias in dGPS positions.

596 Since Method A is based on the position of beams, if the bin position errors related to 597 dGPS accuracy as well as sensor tilt are greater than homogeneity errors associated with 598 beam divergence, standard Method $B$ is more reliable. This is likely to be the case 599 particularly in rivers shallower than those studied here and where high resolution is 600 required due to large velocity gradients. In rivers of the scale studied here, and deeper, 601 by increasing the mesh cell size, we can still have sufficient data to estimate velocity 602 vectors, and the effects dGPS and tilt sensor errors have a minor effect. This confirms the 603 earlier findings by Vermeulen et al., (2014b), which showed that Method A provides the 604 greatest improvement where the aDcp cell size is much smaller than the beam spread. 605 We are not yet in a position to identify the depth at which Method A becomes preferable 606 to Method B, and again this will depend on other parameters such as the intensity of shear 607 and so may not be readily generalizable between confluences.

608 The difficulty of identifying the depths of rivers and intensities of shear that make one 609 method preferable over another precludes adoption of simple quantitative guidance on 610 which method to use when. As both methods have some disadvantages, we argue that 611 both methods should be applied. If they give similar results, then there should be 612 confidence in both. If and where they differ, analysis should be undertaken to identify why, 613 and hence which method is likely to be preferable. Association of the differences in 614 primary and secondary velocities inferred between the two methods with estimates of 
615 shear intensity and with estimated tilt and positioning errors should then help decide 616 whether Method A or Method B is preferable in a particular case. This preference may 617 vary between confluences but also through time at a confluence, if shear or flow depth 618 changes significantly between survey dates.

619 Finally, we wish to emphasise that the impact of averaging is only one element that must 620 be considered in obtaining reliable primary and secondary clow estimates in river 621 confluences. Other issues, such as the rotation method needed to distinguish primary and 622 secondary circulation, remain important and should be considered routinely.

\section{Conclusions}

624 This paper shows the advantage of working with the radial (beam) velocity measurements 625 of an aDcp within each bin prior to averaging them across a given volume of fluid (Method 626 A) as opposed to identify volumes of fluid and assuming bend homogeneity within them 627 (Method B). Such a treatment is important where there are strong velocity gradients in the 628 flow as with river channel confluences. In the first of our case-study confluences, the 629 Lizerne-Rhône, a very small tributary joined the main river, and the pattern of primary and 630 secondary velocities obtained with methods $A$ and $B$ were relatively similar, more so for 631 primary velocities. But for a second confluence, the Grande Eau-Rhône, with a similar 632 momentum ratio, there were much larger differences. We attributed this to the formation 633 of much stronger shear at this confluence. Method A also appeared to reduce the number 634 of repeat transects needed to estimate secondary velocities reliably. The main downside 635 is that Method A is more sensitive to errors related to positioning. Thus, good dGPS 636 accuracy and precision are required to perform a robust estimation of velocity. 
637 In smaller/shallower rivers, Method B may be acceptable indeed preferable as it is less 638 sensitive to GPS errors. In larger rivers, Method A may be necessary, especially in the 639 presence of strong shear at the confluence. Choice between these methods should be 640 based upon an initial screening of the extent to which there is strong shear in the flow as 641 well as the extent to which bins further from the aDcp are influenced by beam divergence.

\section{Appendix A}

643 The LOWESS model is a locally weighted polynomial regression, which at each point and

644 in the range of dataset, a low degree polynomial is fitted to a subset of the data, using 645 weighted least squares. This polynomial fit gives more weight to points closer to the point 646 whose response is being estimated. The value of the regression function for the point is 647 then obtained by evaluating the local polynomial using the explanatory variable values for 648 that data point. The LOWESS fit is complete after regression function values have been 649 computed for each of the $n$ data points. Many of the details of this method, such as the 650 degree of the polynomial model and the weights, are flexible ("Local regression," n.d.).

\section{Acknowledgements}

652 This paper benefited from constructive and detailed suggestions from two anonymous 653 reviewers and Editor Mike Kirkby. The work was supported by Swiss National Science 654 Foundation Grant 200021_160020. The codes used may be obtained upon request to 655 Gelare Moradi. 


\section{References}

657 Alvarez, L. V., Schmeeckle, M.W., Grams, P.E., 2017. A detached eddy simulation model 658 for the study of lateral separation zones along a large canyon-bound river. J. Geophys. 659 Res. Earth Surf. 122, 25-49. https://doi.org/10.1002/2016JF003895.

660 Ashmore, P.E., Ferguson, R.I., Prestegaard, K.L., Ashworth, P.J. and Paola, C., 1992. 661 Secondary flow in coarse-grained braided river confluences. Earth Surface Processes 662 and Landforms, 17, 299-312.

663 Best, J.L. and Roy, A.G., 1991. Mixing-layer distortion at the confluence of channels of 664 different depth. Nature, 350, 411-413.

665 Biron, P., De Serres, B., Roy, A.G., Best, J.L., 1993. Shear layer turbulence at an unequal 666 depth channel confluence. In: Clifford, N.J., French, J.R., Hardisty J. (editors) 667 Turbulence: Perspectives on Flow and Sediment Transport, Wiley, Chichester, 197668213.

669 Dinehart, R.L. and Burau, J.R., 2005. Averaged indicators of secondary flow in repeated 670 acoustic Doppler current profiler crossings of bends. Water Resources Research, 41, $671 \quad$ W09405.

672 Engel, F.L. and Rhoads, B.L., 2016. Three-dimensional flow structure and patterns of bed 673 shear stress in an evolving compound meander bend. Earth Surface Processes and $674 \quad$ Landforms, 41, 1211-1226.

675 Gualtieri, C., lanniruberto, M., Filizola, N., Santos, R. and Endreny, T., 2017. Hydraulic 676 complexity at a large river confluence in the Amazon basin. Ecohydrology, 10, e1863 
677 Gunawan, B., Neary, V.S., McNutt, J.R., 2011. Ornl Adv Post-Processing Guide and 678 Matlab Algorithms for Mhk Site Flow and Turbulence Analysis, Ornl/Tm-2011/404. 679 doi:10.2172/1034377.

680 Jackson, P.R., Garcia, C.M., Oberg, K.A., Johnson, K.K. and Garcia, M.H., 2008. Density 681 currents in the Chicago River: Characterization, effects on water quality, and potential 682 sources. Science of the Total Environment 401: 130-143.

683 Kasvi, E., Vaaja, M., Alho, P., Hyyppä, H., Hyyppä, J., Kaartinen, H. and Kukko, A., 2013. 684 Morphological changes on meander point bars associated with flow structure at 685 different discharges. Earth Surface Processes and Landforms, 38, 577-590.

686 Kasvi, E., Laamanen, L., Lotsari, E. and Alho, P., 2017. Flow Patterns and Morphological 687 Changes in a Sandy Meander Bend during a Flood-Spatially and Temporally Intensive 688 ADCP Measurement Approach. Water, 9, 106.

689 Knox, R. L. and Latrubesse, E.M., 2017. A geomorphic approach to the analysis of 690 bedload and bed morphology of the Lower Mississippi River near the Old River Control 691 Structure. Geomorphology, 268, 35-47.

692 Konsoer, K.M. and Rhoads, B.L., 2014. Spatial-temporal structure of mixing interface 693 turbulence at two large river confluences. Environmental Fluid Dynamics, 14, $1043-$ 6941070.

695 Kostaschuk, R.A., Shugar, D., Best, J.L., Parsons, D.R., Lane, S.N., Hardy, R.J. and 696 Orfeo, O., 2009. Suspended sediment transport and deposition over a dune: Río 697 Paraná, Argentina. Earth Surface Processes and Landforms, 34, 1605-1611. 
698 Lane, S.N., Parsons, D.R., Best, J.L., Orfeo, O., Kostaschuk, R. a., Hardy, R.J., 2008. 699 Causes of rapid mixing at a junction of two large rivers: Río Paraná and Río Paraguay, 700 Argentina. Journal of Geophysical Research, 113, 1-16. 701 https://doi.org/10.1029/2006JF000745.

702 Lane, S.N., Bradbrook, K.F., Richards, K.S., Biron, P.M., Roy, a. G., 2000. Secondary 703 circulation cells in river channel confluences : measurement artefacts or coherent flow 704 structures? Hydrological Processes, 14, 2047-2071. https://doi.org/10.1002/1099705 1085(20000815/30)14:11/12<2047::aid-hyp54>3.0.co;2-4.

706 Local regression [WWW Document], n.d.

707 URL:https://en.wikipedia.org/wiki/Local_regression (accessed 1.22.18).

708 Lotsari, E., Kasvi, E., Kämäri, M., and Alho, P., 2017. The effects of ice cover on flow 709 characteristics in a subarctic meandering river. Earth Surface Processes and $710 \quad$ Landforms, 42, 1195-1212.

711 Marsden, R.F. and Ingram, R.G., 2004. Correcting for beam spread in acoustic Doppler 712 current profiler measurements. Journal of Atmospheric and Oceanice Technology, 21, $713 \quad 1491-1498$.

714 Morlock, S.E., 1996. Evaluation of Acoustic Doppler Current Profiler Measurements of 715 River Discharge. U.S. Geological Survey Water Resources Investigations Report 95$716 \quad 4218$.

717 Muste, M., Yu, K. and Spasojevic M. 2004. Practical aspects of ADCP data use for 718 quantification of mean river flow characteristics: Part I: Moving-vessel measurements. 719 Flow Measurement and Instrumentation, 15, 1-16. 
720 Parsapour-Moghaddam, P. and Rennie, C.D., 2018. Calibration of a 3D Hydrodynamic 721 meandering river model using fully spatially distributed 3D ADCP velocity data. Journal 722 of Hydraulic Engineering, 144, 04018010.

723 Parsons, D.R., Best, J.L., Orfeo, O., Hardy, R.J., Kostaschuk, R.A. and Lane, S.N., 2005. 724 The morphology and flow fields of three-dimensional dunes, Rio Paraná, Argentina: 725 results from simultaneous multibeam echo sounding and acoustic Doppler current 726 profiling. Journal of Geophysical Research, 110, F04S03.

727 Parsons, D.R., Best, J.L., Lane, S.N, Orfeo, O., Hardy, R.J. and Kostaschuk, R.A., 2007. 728 Form roughness and the absence of secondary flow in a large confluence-diffluence, 729 Rio Paraná, Argentina. Earth Surface Processes and Landforms, 32, 155-162.

730 Parsons, D.R., Jackson, P.R., Czuba, J.A., Engel, F.L., Rhoads, B.L., Oberg, K.A., Best, 731 J.L., Mueller, D.S., Johnson, K.K., Riley, J.D., 2013. Velocity Mapping Toolbox (VMT): 732 A processing and visualization suite for moving-vessel ADCP measurements. Earth 733 Surface Processes and Landforms, 38, 1244-1260.

734 Petrie, J., Diplas, P., Gutierrez, M. and Nam, S., 2013. Combining fixed- and moving735 vessel acoustic Doppler current profiler measurements for improved characterization 736 of the mean flow in a natural river. Water Resources Research, 49, 5600-5614.

737 Rennie, C.D., Millar, R.G. and Church, M.A., 2002. Measurement of bed load velocity 738 using an acoustic Doppler current profiler. Journal of Hydraulic Engineering - ASCE, $739 \quad 128,473-483$. 
740 Rennie, C.D. and Millar, R.G., 2004. Measurement of the spatial distribution of fluvial 741 bedload transport velocity in both sand and gravel. Earth Surface Processes and 742 Landforms, 29, 1173-1193.

743 Rennie, C.D., Rainville, F., 2006. Case study of precision of GPS differential correction 744 strategies: influence on aDcp Velocity and discharge estimates. Journal of Hydraulic $745 \quad$ Engineering, 132, 225-234.

746 Rennie, C. D., and Church, M., 2010. Mapping spatial distributions and uncertainty of 747 water and sediment flux in a large gravel bed river reach using an acoustic Doppler 748 current profiler, Journal of Geophysical Research - Earth Surface, 115.

749 Rhoads, B.L. and Kenworthy, S.T., 1995. Field measurements of flow structure at a high750 angle asymmetrical stream confluence. Geomorphology 11, 273-293.

751 Rhoads, B.L. and Kenworthy, S.T., 1998. Time-averaged flow structure in the central 752 region of a stream confluence. Earth Surface Processes and Landforms, 23, 171-191.

753 Rhoads, B.L., and Sukhodolov, A.N., 2001. Field investigation of three-dimensional flow 754 structure at stream confluences: 1 . Thermal mixing and time-averaged velocities. Water $755 \quad$ Resources Research, 27, 2393-410.

756 Rhoads, B.L., and Sukhodolov, A.N., 2004. Spatial and temporal structure of shear layer 757 turbulence at a stream confluence. Water Resources Research, 40, W06304.

758 Rhoads, B.L., and Sukhodolov, A.N., 2008. Lateral momentum flux and the spatial 759 evolution of flow within a confluence mixing interface. Water Resources Research, 44, 760 W08440. 
761 Riley, J.D. and Rhoads, B.L., 2012. Flow structure and channel morphology at a natural 762 confluent meander bend. Geomorphology, 163, 84-98.

763 Riley, J.D., Rhoads, B.L., Parsons, D.R. and Johnson K.K., 2015. Influence of junction 764 angle on three-dimensional flow structure and bed morphology at confluent meander 765 bends during different hydrological conditions. Earth Surface Processes and 766 Landforms, 40, 252-271.

767 Shugar, D.H., Kostaschuk, R., Best, J.L., Parsons, D.R., Lane, S.N., Orfeo, O. and Hardy, 768 R,J., 2010. On the relationship between flow and suspended sediment transport over 769 the crest of a sand dune, Río Paraná, Argentina. Sedimentology 57: 252-272.

770 SonTek YSI, 2000. Acoustic Doppler Profiler (ADP ®) Principles of Operation.

771 SonTek YSI, 2010. RiverSurveyor S5/M9 System Manual 115.

772 Sukhodolov, A.N. and Rhoads, B. L., 2001. Field investigation of three-dimensional flow 773 structure at stream confluences 2. Turbulence. Water Resources Research, 27, 2411 2474.

775 Sukhodolov, A. N., Krick, J., Sukhodolova, T. A., Cheng, Z., Rhoads, B. L. and 776 Constantinescu, G. S., 2017. Turbulent flow structure at a discordant river confluence: 777 Asymmetric jet dynamics with implications for channel morphology. Journal of 778 Geophysical Research: Earth Surface, 122(6), 1278-1293. 779 https://doi.org/10.1002/2016JF004126. 
780 Szupiany, R.N., Amsler, M.L., Best, J.L. and Parsons, D.R., 2007. Comparison of fixed781 and moving vessel measurements with an aDp in a large river. Journal of Hydraulic $782 \quad$ Engineering, 133, 1299-1309.

783 Szupiany, R.N., Amsler, M.L., Parsons, D.R. and Best, J.,L., 2009. Morphology, flow 784 structure, and suspended bed sediment transport at two large braid-bar confluences. $785 \quad$ Water Resources Research, 45, W05415.

786 Szupiany, R.N., Amsler, M.L., Hernandez, J., Parsons, D.R., Best, J.L., Fornari, E. and 787 Trento, A., 2012. Flow fields, bed shear stresses, and suspended bed sediment 788 dynamics in bifurcations of a large river. Water Resources Research, 48, W11515.

789 Tomas, G., Bleninger, T., Rennie, C.D., Guarneri, H., 2018. Advanced 3D mapping of 790 hydrodynamic parameters for the analysis of complex flow Motions in a submerged 791 bedrock canyon of the Tocantins River, Brazil. Water (Switzerland) 10, 1-19. 792 https://doi.org/10.3390/w10040367.

793 Tsubaki, R., Kawahara, Y., Muto, Y. and Fujita, I., 2012. New 3-D flow interpolation 794 method on moving ADCP data. Water Resources Research, 48, 1-15.

795 Venditti, J.G., Rennie, C.D., Bomhof, J., Bradley, R.W., Little, M., Church, M., 2014. Flow 796 in bedrock canyons. Nature 513, 534-537. https://doi.org/10.1038/nature13779.

797 Vermeulen, B., Hoitink, A.J.F., van Berkum, S.W. and Hidayat, 2014a. Sharp bends 798 associated with deep scours in a tropical river: The river Mahakam (East Kalimantan, 799 Indonesia). Journal of Geophysical Research, Earth Surface, 119, 1441-1454. 
800 Vermeulen, B., Sassi, M.G. and Hoitink, A.J.F., 2014b. Improved flow velocity estimates 801 from moving-boat ADCP measurements. Water Resources Research, 50, 4186-4196.

802 Vermeulen, B., Hoitink, A.J.F. and Labeur, R.J., 2015. Flow structure caused by a local 803 cross-sectional area increase and curvature in a sharp river bend. Journal of 804 Geophysical Research, Earth Surface, 120, 1771-1783.

805 Zhao, J., Chen, Z., and Zhang, H., 2014. A robust method for determining the heading 806 misalignment angle of GPS compass in ADCP measurement. Flow Measurements and 807 Instrumentation, 35, 1-10. 
1 "For the ESPL special issue: Measuring and numerical modelling of hydro-

2 morphological processes in open-water"

\section{Evaluation of aDcp processing options for secondary flow}

\section{4 identification at river junctions}

5 Gelare Moradi ${ }^{1}$, Bart Vermeulen ${ }^{3}$, Colin D. Rennie ${ }^{2}$, Romain Cardot ${ }^{1}$, Stuart N. Lane ${ }^{1}$

6 1: University of Lausanne - Institute of Earth Surface Dynamics (UNIL - IDYST)

7 Université de Lausanne - IDYST Quartier Mouline - Bâtiment Géopolis - Switzerland

8 2: Civil Engineering Department, University of Ottawa

9 3: Water Engineering and Management Department, University of Twente, the

10 Netherlands

\section{Abstract}

12 Secondary circulation in river confluences results in a spatial and temporal variation of

13 fluid motion and a relatively high level of morphodynamic change. Acoustic Doppler

14 current profiler (aDcp) vessel-mounted flow measurements are now commonly used to 15 quantify such circulation in shallow water fluvial environments. It is well established that

16 such quantification using vessel-mounted aDcps requires repeated survey of the same 17 cross-section. However, less attention has been given to how to process these data. Most 18 aDcp data processing techniques make the assumption of homogeneity between the 19 measured radial components of velocity. As acoustic beams diverge with distance from 20 the aDcp probe, the volume of the flow that must be assumed to be homogeneous 21 between the beams increases. In the presence of secondary circulation cells, and where 22 there are strong rates of shear in the flow, the homogeneity assumption may not apply, 
23 especially deeper in the water column and close to the bed. To reduce dependence on 24 this assumption, we apply a newly-established method to aDcp data obtained for two 25 medium-sized ( $\sim 60-80 \mathrm{~m}$ wide) gravel-bed river confluences and compare the results with 26 those from more conventional data processing approaches. The comparsion confirms that 27 in the presence of strong shear our method produces different results to more 28 conventional approaches. In the absence of a third set of fully independent data, we

29 cannot demonstrate conclusively which method is best, but our method involves less

30 averaging and so in the presence of strong shear is likely to be more reliable. We conclude

31 that it is wise to apply both our method and more conventional methods to identify where

32 data analysis might be impacted upon by strong shear and where inferences of secondary 33 circulation may need to be made more cautiously.

35 suggests an improvement in secondary flow representation compared to more 36 conventional methods whilst also confirming that repeated transects are required to obtain 37 reliable secondary flow and turbulence measurement. Use of the method resolves two 38 counter-rotating cells in the confluence zone more clearly, with downward velocity in the 39 channel centre. This pattern helps to explain development of confluence scour holes in 40 such streams.

\section{Keywords}

42 Acoustic Doppler current profiler

43 Secondary circulation

$44 \quad$ River confluences 
45 River junctions

\section{Introduction}

47 Acoustic Doppler current profilers (aDcps) are now used widely to measure river flow in 48 three-dimensions, notably for the quantification of secondary flows. Applications have 49 been made to river bedforms (e.g., Parsons et al., 2005; Kostaschuk et al., 2009; Shugar 50 et al., 2010), bends (e.g., Dinehart and Burau, 2005; Kasvi et al., 2013; Vermeulen et al., 51 2014a, 2015; Engel and Rhoads, 2016; Knox and Latrubesse, 2016; Kasvi et al., 2017; 52 Lotsari et al., 2017; Parsapour-Moghaddam and Rennie, 2018), junctions (e.g., Parsons 53 et al., 2007; Lane et al., 2008; Szupiany et al., 2009; Riley and Rhoads, 2012; Riley et al., 54 2015; Gualtieri et al., 2017), bifurcations (e.g., Parsons et al., 2007; Szupiany et al., 55 2012), canyons (e.g., Alvarez et al., 2017; Tomas et al., 2018; Venditti et al., 2014), deltas 56 (e.g., Czuba et al., 2011) and gravity currents (e.g., Garcia et al., 2007; Garcia et al., 57 2012). Research has also shown the need to make repeat section measurements (e.g., 58 Szupiany et al., 2007; Jackson et al., 2008) and also to process these data carefully, 59 (Muste et al., 2004; Rennie and Church 2010; Tsubaki et al., 2012; Parsons et al., 2013; 60 Petrie et al., 2013). Such processing must take into account positioning (Rennie and 61 Rainville, 2006) and orientation (Zhao et al., 2014) errors, and the treatment of repeat 62 section measurements (e.g., Szupiany et al., 2007; Jackson et al., 2008).

63 This paper is concerned with recent observations regarding the inference of secondary 64 flows from aDcp data and concerns regarding the assumption that flow is homogenous in 65 the fluid volumes defined by the acoustic beams emitted from an aDcp and used to 66 calculate any one point estimate (Vermeulen et al., 2014b). Acoustic beams are reflected 
67 by suspended particles, which, if moving, cause a Doppler shift in beam frequency, which

68 is then detected at the sensor. This shift is directional so each beam measures the radial 69 velocity, which is the velocity of particle motion parallel to the acoustic path. This can be 70 assumed to be the flow velocity if the particle motion is identical to fluid motion. In order

71 to resolve flow in more than one direction, aDcps require at least three acoustic beams to

72 estimate three Cartesian components of velocity. The radial velocities originating from the

73 beams are traditionally analyzed for a single measurement cycle at a single depth at a

74 time (Vermeulen et al., 2014b). The velocity then applies to the volume of fluid defined by

75 the beams at each depth. Flow within this volume is assumed to be homogeneous.

76 However, as the beams spread from the sensor, depth bins increase in horizontal size

77 (Rennie et al., 2002). This means that: (1) bins further from the sensor are likely to produce

78 less reliable velocities because the bin size is greater and the flow within bins is more

79 likely to be heterogeneous (Gunawan et al., 2011); and (2), even in smaller bins, velocities

80 may be less reliable in zones of strong shear where also the within-bin flow is less likely

81 to be homogeneous. In a river where measurements are made throughout the flow depth,

82 the maximum shear may be close to the bed, where the beam divergence may also be 83 greatest.

84 One solution to this problem accounts for first order shear within the flow volume (e.g.

85 Marsden and Ingram, 2004) through a Taylor expansion of the coordinate transform used

86 to determine the Cartesian velocity components. Under this solution, flow is allowed to

87 vary linearly within the bin, but the bin's volume becomes potentially larger with distance

88 from the sensor. Vermeulen et al. (2014b) developed and tested a second solution. As

89 explained in detail below, multiple radial (beam) velocity measurements within a single bin 
90 are put through a Cartesian transform to obtain a localized within-bin three-dimensional 91 velocity. This method strongly reduces the volume over which homogeneity should be 92 assumed and Vermeulen et al. (2014b) found that this significantly impacted 93 interpretations of secondary velocities in the presence of strong shear. In this paper, we 94 seek to quantify the effects of this method for the measurement of secondary flow in two 95 medium-sized river junctions (c. $60-80 \mathrm{~m}$ post-junction channel width). River junctions are 96 associated with very strong shear (e.g. Best and Roy, 1991; Biron et al., 1993, 1996a, 97 1996b; Sukhodolov and Rhoads, 2001; Rhoads and Sukhodolov, 2004, 2008; Konsoer 98 and Rhoads, 2014; Sukhodolov et al., 2017), as well as well-developed secondary 99 circulation (e.g. Ashmore et al., 1992; Rhoads and Kenworthy, 1995, 1998; Rhoads and 100 Sukhodolov et al., 2001; Lane et al., 2008; Riley and Rhoads, 2012; Riley et al., 2015). 101 Thus, understanding how to process effectively the aDcp data used to describe them is of 102 paramount importance.

\section{Methods for estimating Cartesian velocity components from aDcp data}

104 In this section, we describe the two different methodological approaches used in this study 105 to estimate Cartesian velocity components: (1) Mmethod A, the Vermeulen et al., (2014b) 106 method; and (2) Mmethod B, the conventional method. Common to all methods is the 107 assumption that data are available from repeat measurement of the same cross-section, 108 as has been shown to be critical for obtaining reliable estimates of secondary circulation 109 from aDcp data (Szupiany et al., 2007), particularly when single transect measurements 110 are not close enough together. 


\section{Method A: based on Vermeulen et al., (2014b)}

112 Application of the Vermeulen et al. (2014b) method requires mapping of radial beam 113 velocity data onto a predefined mesh. This mesh requires both a bottom topography or

114 bathymetric model, and an upper limit just below the water surface. As the measurements 115 were made using several repeat transects for each cross section, the first step is to define 116 a mean cross section for each set of individual transects (boat tracks). The second step 117 is to define a grid mesh for this mean cross section. Third, all measured beam velocities 118 are projected on to this cross section mesh. Finally, the beam velocities within each mesh 119 grid-cell are then used to resolve a Cartesian velocity for the meshgrid cell. Errors that 120 influence these steps can be estimated.

121 The first step is estimation of the mesh extremes, both the lower boundary or bathymetry 122 model and the upper boundary near the water surface. To generate the bathymetry model 123 we use depth soundings collected with the aDcp. We recognize that each beam may 124 register a different distance of the stream bed from the sounder, especially as we are 125 dealing with bathymetricallyłargely irregular cross-sections. Specifically, for each bottom 126 track sounding within each transect, we use the UTM coordinates obtained with a coupled 127 differential GPS (dGPS), the range of each bottom track beam return, and the instrument 128 tilt to estimate the bed elevation and horizontal position of each beam impingement point 129 on the bed. These bed positions are combined together to identify an initial mean transect. 130 Provided a point is within a certain distance from the initial mean cross-section, LOWESS 131 interpolation (Appendix A) is applied, which has the effect of defining a bathymetric model 132 that gives most weight to points that appear to be closer to the cross-section. It is important 133 to note that this mean transect is not necessarily orthogonal to the primary flow direction 
134 and so will not yield true primary and secondary flow estimates without further correction.

135 We address this below.

136 Once the initial bathymetric model is defined, we estimate a unique vector using the initial 137 mean transect; that is the principal direction of the scatter cloud of all $\mathrm{x}$ and $\mathrm{y}$ UTM 138 positions at the bed. This unique vector points in the direction of the largest eigenvector 139 of the covariance matrix of all UTM positions $(t)$. We then calculate the mean UTM position $140\left(p_{\text {mean }}\right)$ for each set of individual transects and the difference between each measured 141 beam position $\left(p_{b}\right)$ and the mean position. The dot product of these obtained values and 142 the unique vector is then used to define the projection of each UTM position in the direction 143 of the unique vector. To identify the final mean cross section, we sum up all individual 144 projected vectors and obtain the best fit to all available data (Figure 1).

145 To define the upper boundary of the mesh, we estimate the elevation of the water surface.

146 As there is a blanking distance at the surface of the water during the measurement, we

147 then remove this blanking distance, taken as $0.30 \mathrm{~m}$. Thus, the mesh has also a blanking

148 distance and the upper part of the cross-section is, strictly, the upper limit of available 149 data, not the water surface.

150 "Figure1"

151 The second step uses the defined bathymetric model and available velocity bins within 152 the measured area (not influenced by side lobes, and below the blanking distance) to 153 define a cross-section mesh. The side-lobe interference is caused by the striking of the 154 channel bed by side-lobe energy from each of the acoustic beams. This side-lobe energy 155 has strong reflections from the bed, which result in echoes that overwhelm the signal from 
156 scatters near the bed. The thickness of the side-lobe layer is typically $6-7 \%$ of the 157 measured depth (Morlock, 1996).

158 To generate the mesh, the cross section is initially subdivided into vertical slices with equal 159 widths $(\Delta n)$. For each slice, the simplest definition of mesh cell thicknesses $(\Delta z)$ divides 160 each vertical equally. These verticals are converted to non-dimensional $\sigma$ coordinates 161 using following equation:

$162 \sigma=1-\left(\frac{p_{v} \cdot \boldsymbol{k}-\eta}{p_{b} \cdot \boldsymbol{k}-\eta}\right) \quad$ (Vermeulen et al. 2014b)

163 where $p_{v}$ stands for velocity measurement positions $(\mathrm{m}), p_{b}$ is the corresponding bed 164 position $(\mathrm{m})$ that is found using velocity measurement horizontal positions and applying 165 the bathymetric model, $\boldsymbol{k}$ is the upward pointing unit vector and $\eta$ are the water surface 166 fluctuations around the mean water level at which $z=0$.

167 However, because of beam spreading and differences in the distance of the sounder from 168 the bed, which varies with position of the sounder, this tends to produce a highly 169 heterogeneous number of measurements in each cell within the mesh. The alternative, 170 adopted here, is to allow mesh cell thickness to vary through the water column such that 171 there is a roughly equal number of beam velocities contributing to each mesh cell (see 172 Figure 2 for a typical distribution).

173 As the river bed form is varying, to follow its shape, each mesh cell is considered to be a 174 cuboid with 6 edges, two on the left side, two in the middle and two on the right side. To 175 define these edges, the first step is to define the middle point of each mesh cell. Once 176 defined, by calculating the slope for each half part of the mesh cell, edges can be obtained. 
177 The mesh cell faces are then calculated on the basis of adjacent verticals and the mesh 178 cell upper and lower boundaries.

179 To identify the beams that contribute to each mesh cell, an index for each beam velocity 180 is defined, which shows its associated mesh cell, using the projection of each radial 181 velocity onto the estimated mean cross section (Figure 2).

182 "Figure 2"

183 In the third step, the radial velocities for each beam (b) that contribute to each mesh cell 184 (the $\mathrm{N}$ beam velocities) have to be transformed into Cartesian velocities $\left(v_{x}, v_{y}\right.$ and $\left.v_{z}\right)$ 185 using:

$186 \quad\left(\begin{array}{c}b_{1} \\ \vdots \\ b_{N}\end{array}\right)=\left(\begin{array}{c}q_{1} \\ \vdots \\ q_{N}\end{array}\right) \cdot\left(\begin{array}{c}v_{x} \\ v_{y} \\ v_{z}\end{array}\right) \leftrightarrow b=Q \cdot u$

187 where $\mathbf{q}$ is a unit vector which describes the direction of the acoustic beam.

188 To obtain the raw beam velocities, we use matrix transformations obtained from the raw 189 data to transform measured velocities in $\mathrm{XYZ}$ coordinates into beam velocities. The 190 Vermeulen et al., (2014b) method includes in the transformations an explicit treatment of 191 the random errors due to internal and external factors and the bias (systematic errors) 192 caused by the measurement system and the nature of river flow (Tsubaki et al., 2012).

193 Random errors include those that come from sampling a time-varying flow in the presence 194 of strong gradients and represent a form of aliasing. By adding a combined term of errors $195 \varepsilon,(2)$ becomes:

$196 \mathbf{b}=\mathbf{Q u}+\varepsilon$ 
197 A least squares solution is fitted to (3) that minimizes the sum of the square of the errors.

198 The optimal estimation $(\hat{\mathbf{u}})$ for $(\mathbf{u})$ is then given by the normal equation:

$199 \hat{\mathbf{u}}=\mathrm{Q}^{+} \mathbf{b}+\varepsilon$

200 where $\mathrm{Q}^{+}$can be defined as:

$201 Q^{+}=\left(Q^{\top} Q\right)^{-1} Q^{\top}$

202 To solve three Cartesian velocity components, we need at least three equations. Each 203 beam measurement in a mesh cell adds an equation. Where enough beam velocities are 204 collected in a mesh cell and the equations are different from each other (beam velocities 205 are measured from different directions), the velocity can be estimated. To check whether 206 this is the case, the matrix describing the system of equations can be analyzed. In the 207 processing we use the rank which indicates how many unknowns can be solved from the 208 system of equations. When the rank is three, the three Cartesian velocities can be solved. 209 Where the rank of the matrix is one or two, the system cannot be solved. Where the 210 system of equations is overdetermined, the obtained solution is a matrix with more 211 equations (rows) than unknowns (columns). The velocity can be solved using the 212 generalized inverse of the matrix and in such a way that the sum of squared errors is 213 minimized. As this combined term of errors also contains information about the turbulence 214 and accuracy of the measurements, we can obtain the covariance matrix of the velocity 215 components:

$216 \hat{\varepsilon}=\mathbf{b}-Q \hat{u}$

$217 \operatorname{var}(\hat{\mathbf{u}})=\frac{\hat{\varepsilon}^{\top} \hat{\varepsilon}\left(Q^{\top} Q\right)^{-1}}{\mathrm{~N}-3}$ 
218 and the variance of the velocity across the section can be then estimated as:

$219 \operatorname{var}(\mathbf{u})=\frac{\operatorname{var}(\hat{\mathbf{u}})}{\mathrm{N}}$

$220 \quad(8)$

\section{Method B: the standard aDcp method}

222 As the Doppler shift is directional, it can only measure radial velocities. With the standard 223 method, to determine Cartesian velocity components, radial velocities then have to be 224 resolved into three orthogonal velocity vectors. To do so, at least three beam velocities 225 pointed in known directions are required. Also, because the beams are measuring 226 different water profiles along their individual slant ranges, the assumption of horizontal 227 homogeneity must be taken into account. Hence, in the standard method, the three 228 dimensional velocity for each depth bin for each ping can be solved for a typical four-beam 229 system using the following equations (Mueller and Wagner, 2009):

$230 \quad V_{x}=\frac{\left(b_{3}-b_{1}\right)}{\sqrt{2} \sin \theta}$

$231 \quad V_{y}=\frac{\left(b_{4}-b_{2}\right)}{\sqrt{2} \sin \theta}$

$232 \quad V_{z}=\frac{-\left(b_{1}+b_{3}\right)}{(2 \cos \theta)}=\frac{-\left(b_{2}+b_{4}\right)}{(2 \cos \theta)}$

$233(11)$

234 where $V_{y}$ is the cross stream velocity assuming beam 3 is pointed upstream, $V_{x}$ is the 235 streamwise velocity, $V_{z}$ is the vertical velocity, $b_{1}, b_{2}, b_{3}$ and $b_{4}$ are the radial velocities 236 measured in beams $1,2,3$ and 4 respectively and $\theta$ is the tilt angle of the beams referenced 237 to vertical. These data should then be corrected for pitch and roll angles, obtained from 
238 the internal inclinometer and the heading angle from the internal compass. Velocity 239 outputs are already corrected for ship velocities.

240 To compare results obtained using $\underline{M}$ method $B$ with those of $\underline{M}$ method $A$, we use the same

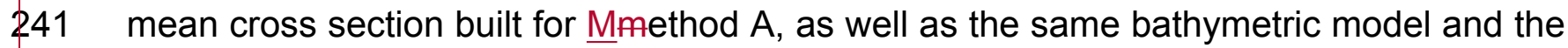
242 same mesh. Each measured velocity vector is assigned to the appropriate mesh cell by 243 projecting its 3D position (horizontal position and depth) onto the mean cross section 244 mesh. We then average $x, y$, and $z$ components of all velocities measured within a mesh 245 cell to obtain the mean velocity vector for the mesh cell.

\section{Methodology}

247 This paper is motivated by the need to acquire three-dimensional data from junctions of 248 tributaries with a main river stem, here the River Rhône, western Switzerland, and so the 249 need to identify methods for reliably obtaining Cartesian velocities from aDcp data. The 250 Rhône tributaries typically have very high bedload transport rates for short periods of time, 251 leading to the formation of very large tributary mouth bars downstream of their junctions 252 with the main river. These bars are maintained for weeks or months such that at lower 253 tributary flow, with negligible sediment supply, there is a legacy effect of previous high 254 momentum tributary events upon junction morphology and secondary flow formation.

255 For this paper, we used a specially-designed rope and pulley system to collect aDcp data 256 from the junction of two tributaries with the Rhône (Figure 3).

257 "Figure 3" 
258 The Lizerne is a Rhône tributary of almost $20 \mathrm{~km}$ length that flows south-westward from 259 the western slopes of the Tête Noire $(2451 \mathrm{~m})$ or La Fava $(2612 \mathrm{~m})$, in the Bernese Alps. 260 This river is heavily regulated for hydropower with sediment extracted upstream of the 261 junction. As a result, there is negligible sediment supply and no evidence of point bar 262 formation. It reaches the Rhône between Ardon and Vétroz, forming a $90^{\circ}$ junction angle 263 and it has a bed that is nearly concordant with the Rhône.-

264 The Grande Eau is a second tributary of the Rhône River which has a length of $26 \mathrm{~km}$ and 265 takes its source on the Vaud side of the Les Diablerets and flows into the Rhône River 266 with a $70^{\circ}$ confluence angle, near Aigle. The Grande Eau bed is $1.5 \mathrm{~m}$ higher than the 267 Rhône such that it is markedly discordant. It has a catchment area of $132 \mathrm{~km}^{2}$-and the 268 maximum monthly runoff occurs in May with an average of $52.5 \%$ of total annual runoff 269 occurring during snowmelt in the four months April-duly.

270 In this section, we: (1) describe the aDcp used to collect data; (2) describe how the aDcp 271 was deployed; and (3) outline the analytical approaches used to interpret the results from 272 the different methods. Although the method is valid for any aDcp that has an onboard 273 compass and potential for differential GPS positioning, as is standard with most aDcps, 274 we use a Sontek M9 aDcp in this study.

\section{The Sontek M9 aDcp}

276 The SonTek M9 aDcp is a nine-transducer system with three acoustic frequencies, 277 configured as two sets of four profiling beams $(3 \mathrm{MHz}$ and $1 \mathrm{MHz}$ transducers in Janus 278 configurations) and one vertical beam (0.5 MHz Echo sounder) for depth measurements 279 (SonTek YSI, 2010). It uses these two sets of four beams to provide raw radial velocity 
280 samples. These beams are equally spaced at $90^{\circ}$ azimuth angles and are projected at an 281 angle $\theta$ of $25^{\circ}$ off the vertical axis (SonTek YSI, 2000). For the standard configuration, 282 the four beams encompass a sampling diameter of $93 \%$ of the distance from the aDcp 283 (7\% of side-lobe) (SonTek YSI, 2000).

284 The output velocities from the SonTek M9 Riversurveyor are either in Cartesian 285 coordinates (XYZ) that are relative to sensor orientation or in Earth coordinates (ENU) for 286 a SonTek system with compass and tilt sensors. These raw velocity data in Earth 287 coordinates or $\mathrm{XYZ}$ coordinates are already corrected for the ship motion. To apply 288 Mmethod A to Sontek output data, as this method is based on radial velocities, it is 289 necessary to transform these output velocities to radial velocities. To do so, we add ship 290 velocities to these output velocities and then apply the inverses of the instrument's matrix 291 coordinate transformations (obtained from MATLAB files output by the SonTek data 292 collection software RiverSurveyor). As the survey is being undertaken using a moving 293 vessel, these radial velocities then have to be corrected again for the boat velocity. There 294 are two key methods for doing this. The first uses the bottom tracking to measure the boat 295 velocity relative to the river bed, under the assumption that the latter is stationary (i.e. 296 there is no bedload transport). The second tracks the boat position using differential GPS 297 (dGPS, e.g. Zhao et al., 2014). In this study, we corrected all raw beam velocities for ship 298 velocities, using dGPS as we could not exclude the possibility of there being bedload 299 transport.

300 To apply Mmethod B in this study, we use the raw velocity data in Earth coordinates and 301 we correct it for pitch and roll angles, obtained from internal inclinometer and heading 
302 angle data for the internal compass. For SonTek M9 aDcps, pitch is a y-axis rotation and 303 roll an $\mathrm{x}$-axis rotation.

304 Depending on the water depth and velocity, the Sontek M9 firmware changes the acoustic 305 operating frequency and the water profiling mode on-the-fly, thus the number of measured 306 cellssampled points in the vertical varies automatically from one profile to the next. 307 Specifically, when the water is shallower than $0.75 \mathrm{~m}$ and the maximum velocity is less 308 than $0.4 \mathrm{~ms}^{-1}$, the $\mathrm{M} 9$ reports data acquired with a $3 \mathrm{MHz}$ frequency using the pulse 309 coherent mode to obtain a $2 \mathrm{~cm}$ depth measurement resolution. For deeper situations, this 310 frequency changes to $1 \mathrm{MHz}$ pulse coherent pings using a $6 \mathrm{~cm}$ aDcp cell size. If the 311 maximum velocity is greater than $0.4 \mathrm{~ms}^{-1}$ then SmartPulse (i.e., broadband) mode is 312 utilized, with the $3 \mathrm{MHz}$ beams if depth is less than $5 \mathrm{~m}$ and the $1 \mathrm{MHz}$ beams if depth is 313 greater than $5 \mathrm{~m}$, with the aDcp cell size optimized based on the current water depth. As 314 a result of these on-the-fly changes, each measured profile has a different number of aDcp 315 cells and different aDcp cell sizes. Hence, to correct the aDcp cell size variability, for both 316 mMethods $A$ and $B$ there is the need to define a cross-sectional mesh and to project the 317 measured velocities to this mesh. For Method A we use the beam velocity vertical 318 positions in a non-dimensionalized coordinate system using equation 1 , within the 319 predefined mesh explained in section 2.1.

\section{Deployment of the Sontek M9 in the river junctions}

321 The survey work was undertaken in two junctions of the Swiss River Rhône, the Lizerne322 Rhône confluence in August 2017 and the Grande Eau-Rhône confluence in May 2018, 323 using a Sontek M9 vessel mounted aDcp and a specially-designed rope-pulley system 324 (Figure 3c). The survey was spatial, monitoring 11 cross-sections from upstream of the 
325 junction to its downstream at the Lizerne-Rhône confluence with a Momentum ratio (Mr)

326 of 0.018 (Figure 3a) and 11 cross-sections at the Grande Eau-Rhône confluence with a $327 \mathrm{Mr}$ of 0.022 (Figure $3 \mathrm{~b}$ ). Table 1 shows the general characteristics of these two 328 confluences on the date of the measurements.

329 "Table 1"

330 As proposed previously by Dinehart and Burau (2005), Szupiany et al. (2007), Gunawan 331 et al. (2011) and Vermeulen et al., (2014b) at least five repeats are required to have a 332 robust estimation of secondary velocities. Hence, in this paper, data are processed for 333 cross-section 6 at the Lizerne-Rhône confluence (Figure 3a), and for cross section 3 at 334 the Grande Eau-Rhône confluence (Figure 3b). Identification of the minimum number of 335 repeat transects necessary per cross-section was undertaken using cross-section 9 at the 336 Lizerne-Rhône confluence (Figure 3a), which involves 16 repetitions. We noted that after 337 application of Method A, the standard deviation of velocity stabilized with six repetitions, 338 which is the number we adopt for this study.Hence, in this paper, data are processed for 339 cross-section 9, which involves 16 repeat transect surveys (Figure $3 a$ ) at the Lizerne340 Rhêne confluence and for cross section 3 , which involves 6 repeat transect surveys 341 (Figure 3b) at the Grande Eau-Rhône confluence. The decision to use fewer repeat 342 transects at the Grande Eau-Rhône was based upon the identification of the minimum 343 number of cross-sections needed from the Lizerne-Rhône study.

\section{Bin position error determination}

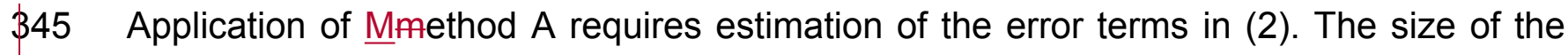
346 sampling volume in each beam is determined by the size of the bin used. As the SonTek 
347 M9 aDcp uses different bin sizes depending on the water track frequency (section 2.1.3), 348 these volumes could vary. Applying Mmethod A might improve the velocity estimation for 349 large measurement volumes at depth, as it does not rely on the homogeneity assumption.

350 But as bins with a small number of velocity measurements will have greater error, this 351 method can estimate velocities with error. Also, if the beam velocity distribution within 352 each mesh cell is not linear, as averaging is made in the middle of each mesh cell, it can 353 introduce error in velocity estimation. Thus, it is necessary to calculate a minimum 354 necessary mesh cell size when applying Mmethod A.

355 Method B is inherently limited by spatial averaging due to the potential use of divergent 356 beams and the associated homogeneity assumption. In other words, one must assume 357 that the velocity is homogeneous over the horizontal domain defined by beam divergence 358 (Eq.12). Method A has the advantage that velocities are recorded within an individual 359 beam depth bin, thus no spatial averaging between beams is required. However, in order 360 for Mmethod A to overcome the uncertainty induced by spatial averaging inherent to 361 Mmethod B, it is essential that the bin location is known explicitly. Error in bin location can 362 be induced by dGPS position and or tilt sensor (pitch and roll) errors. We therefore 363 compare possible bin position errors using Mmethod A to beam divergence obtained from 364 Mmethod B to indicate when Mmethod A should be advantageous over Mmethod B.

365 Beam divergence is the spatial separation of the beams due to the Janus configuration of 366 the beams with beam angles of $25^{\circ}$. This divergence determines the sampling volume that 367 must be considered homogeneous for Method B and can be calculated using equation 368 12:

$369 x_{b}=2 d \tan \theta$ 
370 where $d$ is the depth in $m$ and $\theta$ is the beam angle which for a SonTek aDcp is $25^{\circ}$. The 371 aDcp dGPS is used to reference the velocity measurements in space and to estimate the 372 ship velocity. If dGPS is used for ship velocity, this introduces errors in measurement of 373 the absolute water velocity (because ship velocity is subtracted from the water velocity 374 measured in the reference frame of the aDcp). This uncertainty introduces error in velocity 375 calculations.

376 To estimate the errors due to dGPS and the tilt sensors, in this study we assume normally 377 distributed random errors with a standard deviation of $\pm 1^{\circ}$ for tilt sensors, based on 378 manufacturer specifications, and a normally distributed displacement error for-measured 379 by the dGPS for the dGPS positions (as a function of satellite configuration during 380 measurement), and we apply a Monte Carlo approach which we run 100 times sampling 381 under these uncertainties. Each time we calculate the estimated secondary velocity 382 differences as compared with the original secondary velocities.

383 To be able to reduce the uncertainty due to velocity estimation using Mmethod $A$ 384 compared to $\underline{M} m e t h o d B$, the errors induced in Mmethod A related to GPS uncertainty 385 and tilt sensors must be less than the errors in Mmethod $B$ due to beam divergence and

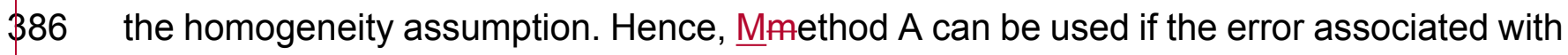
387 a minimum aDcp cell size is in between the error due to beam divergence and the 388 maximum estimated error due to the GPS and tilt sensors. Otherwise using this method 389 introduces more error in velocity estimations than using Mmethod B.

\section{Data interpretation}

391 Methods A and B, described above, were applied to the Sontek M9 data, to determine 392 Cartesian velocities $\left(v_{x}, v_{y}\right.$ and $\left.v_{z}\right)$. As our interest is in process estimation, here we 
393 describe the methods we apply to the Cartesian velocities to estimate processes relevant 394 to junction dynamics. In order to distinguish between primary and secondary components 395 of flow, we need to rotate the initial mean transect. Options for doing this are reviewed in 396 Lane et al. (2000) and we do not assess them here, but rather applyapply the zero net 397 cross stream discharge definition (Lane et al., 2000). By calculating the mean values of 398 the $x$ and $y$ velocity components $(U$ and $V)$, we then calculate the velocity magnitude $(v)$. 399 By rotating these velocity components to the direction of the cross-stream velocity, using 400 the unique vector $(\boldsymbol{\sigma})$, primary velocity vectors $\left(\boldsymbol{v}_{\boldsymbol{p}}\right)$ and secondary velocity vectors $\left(\boldsymbol{v}_{\boldsymbol{s}}\right)$ 401 then can be estimated.

$402 \quad v=\sqrt{U^{2}+V^{2}}$

$403(13)$

$404\left(\begin{array}{l}\sigma_{x} \\ \sigma_{y}\end{array}\right)=\left(\begin{array}{l}U \\ V\end{array}\right) / v$

405 where $\sigma_{x}$ and $\sigma_{y}$ are sin and cos of the angle between the section angle and east.

$406 \quad \boldsymbol{v}_{p}=\sigma_{x} \boldsymbol{v}_{x}+\sigma_{y} \boldsymbol{v}_{y}$

$407 \quad \boldsymbol{v}_{s}=-\sigma_{y} \boldsymbol{v}_{x}+\sigma_{x} \boldsymbol{v}_{y}$

408 However, secondary circulation is all flow that is orthogonal to the primary flow and not 409 just horizontal flow; there should be not net secondary flux in a section; and so correction 410 should also consider vertical velocities. this rotation does not account for the possibility 411 that there is net vertical motion in a section, which is also a component of secondary 412 eirculation. Thus, we extend these relationships to include vertical velocities: 
$413\left(\begin{array}{lll}\sigma_{x, 1} & \sigma_{x, 2} & \sigma_{x, 3} \\ \sigma_{y, 1} & \sigma_{y, 2} & \sigma_{y, 3} \\ \sigma_{z, 1} & \sigma_{z, 2} & \sigma_{z, 3}\end{array}\right)=\left(\begin{array}{c}U \\ V \\ W\end{array}\right) / v$

414 where: $U, V$ and $W$ are the mean velocities of $x, y$ and $z$ velocity components, respectively

415 and $v$ is the magnitude of the velocity which can be obtained using:

$416 \quad v=\sqrt{U^{2}+V^{2}+W^{2}}$

$417 \quad \boldsymbol{v}_{p}=\sigma_{x, 1} \boldsymbol{v}_{x}+\sigma_{x, 2} \boldsymbol{v}_{y}+\sigma_{x, 3} \boldsymbol{v}_{z}$

418 (19)

$419 \quad \boldsymbol{v}_{s}=\sigma_{y, 1} \boldsymbol{v}_{x}+\sigma_{y, 2} \boldsymbol{v}_{y}+\sigma_{y, 3} \boldsymbol{V}_{z}$

$420(20)$

$421 \quad \boldsymbol{V}_{v}=\sigma_{z, 1} \boldsymbol{V}_{x}+\sigma_{z, 2} \boldsymbol{V}_{y}+\sigma_{z, 3} \boldsymbol{V}_{z}$

$422(21)$

423 To estimate velocity gradients, and to correct for weak curvature with the survey method

424 at the edges of each transect line (e.g. Figure 3), solve the curvature of cross sections,-all 425 data have been transformed into row and column coordinates ( $\eta$ and $\zeta$ ) using the following 426 transformation:

$427 \quad\left(\begin{array}{l}\frac{\partial}{\partial n} \\ \frac{\partial}{\partial z}\end{array}\right)=\left(\begin{array}{ll}\frac{\partial \eta}{\partial n} & \frac{\partial \zeta}{\partial n} \\ \frac{\partial \eta}{\partial z} & \frac{\partial \zeta}{\partial z}\end{array}\right)\left(\begin{array}{c}\frac{\partial}{\partial \eta} \\ \frac{\partial}{\partial \zeta}\end{array}\right)$

428 where $n$ and $z$ are horizontal and vertical coordinates on the section plane, respectively 429 (Vermeulen et al., 2014b). 


\section{Results}

\section{$431 \quad$ Primary and secondary velocities}

432 Primary and secondary velocities estimated using mmethods $A$ and $B$ for the Lizerne433 Rhône confluence appear to be similar at cross-section 6 (Figures $4 a$ and $4 b$ ) and the 434 differences in estimated secondary flows are minor. The differences are most pronounced 435 bBetween --10 and $-5 \mathrm{~m}$, in the middle of the main channel, 436 velocity vectors are more pronounced.

437 These primary and secondary velocity patterns show higher differences at cross-section 4383 of the confluence of Grande Eau-Rhône (Figures 4c and 4d) despite it having a similar 439 momentum ratio to the Lizerne during measurement. Primary velocities differ significantly 440 between mmethods A and B: (1) at greater distance from the aDcp because the bins 441 contain larger volumes of water assumed to be homogenous; and (2) at the edges of the 442 cross-section where there are more beam velocity measurements (contours in Figures 4c 443 and 4d). Secondary velocity vectors estimated using Mmethod A indicate flow 444 convergence at the surface and flow descending towards the riverbed throughout the 445 centre of the channel (Figure 4c). This is due to a high degree of bed discordance between 446 the Grande Eau and the Rhône, which increases the penetration of the tributary flow into 447 the main channel above-over the junction, which-and which forms a zone of high lateral 448 and vertical shear, on the one hand, and main channel narrowing because of penetration 449 of the tributary point mouth bar on the other hand. The secondary velocity vectors 450 estimated by $\underline{M}$ method B show a weaker penetration of the tributary flow into the main 451 channel, which results in a reverse flow towards the bank on the inner tributary bank $\underline{\text { side }}$ 452 of the channel at the surface of the mixing interface (Figure 4d). In this case, the core of 
453 the secondary circulation is located in the middle of the main channel and closer to the 454 inner bank.

455 "Figure 4"

456 Figure 5 and Figure 6 quantify the differences in primary and secondary velocity patterns 457 estimated using mmethods $\mathrm{A}$ and $\mathrm{B}$, for the Lizerne-Rhône confluence. Figures $5 \mathrm{a}$ and $4585 \mathrm{c}$ and Figures $6 \mathrm{a}$ show that almost $4 \%$ of mesh cells have a relative difference in primary 459 velocities between mmethods $A$ and $B$ of more than $10 \%$. These differences can exceed $460 \quad 0.2 \mathrm{~ms}^{-1}$ and so they are relatively small. Velocity differences are more pronounced in 461 estimated secondary velocities, with almost $82 \%$ of mesh cells having a difference of more 462 than $10 \%$, and almost $37 \%$ of mesh cells having a difference of more than $50 \%$ (Figure $4635 b, 5 d$ and $6 b)$.

464 "Figure 5"

465 "Figure 6"

466 At the Grande Eau-Rhône confluence, these differences are greater as compared with 467 those of the Lizerne-Rhône confluence. Figures 7a, 7c and 8a show that these differences 468 for primary velocities exceed $0.4 \mathrm{~ms}^{-1}$ in the zone of high vertical and lateral shear and 469 near the inner bank. Almost $20 \%$ of the mesh cells have a relative difference in primary 470 velocities between mmethods $A$ and $B$ of more than $10 \%$. The secondary velocity 471 differences are more pronounced between these two methods. Figures $7 \mathrm{~b}$ and $7 \mathrm{~d}$ show 472 differences with a magnitude of $0.4 \mathrm{~ms}^{-1}$ near the edges and near the bed. Almost all the 473 mesh cells have a difference in estimated secondary velocities between two methods. 474 Figure $8 \mathrm{~b}$ shows that almost $93 \%$ of the mesh cells have a relative difference of $10 \%$ 
475 between mmethods $A$ and $B$. although this value decreases to $55 \%$ for a relative 476 difference of $90 \%$ between these two methods.

477 "Figure 7"

478 "Figure 8"

\section{Velocity gradients}

480 As Figure 9 shows, there is a strong relationship between lateral gradient in secondary 481 velocities and differences between the secondary velocities estimated using methods $A$ 482 and B for both the Lizerne-Rhône and the Grande Eau-Rhône confluences. This is 483 because a stronger velocity gradient increases the probability that the assumption of flow 484 homogeneity within a bin is likely to fail. Indeed, the marked differences between methods $485 \mathrm{~A}$ and $\mathrm{B}$ at the Grande Eau confluence (Figure 7) in the true right secondary circulation 486 cell describe above areis also in a zone of strong lateral shear.

487 "Figure 9"

488 Number of repeat transects

489 One way to reduce data fluctuations due to random errors and turbulence, during the 490 measurement using moving vessel aDcps, is to average by using several repeat transects 491 together in one cross section. As each estimated velocity measurement is a single sample 492 in time, adding in a repeat section adds in an additional estimated velocity measurement. 493 Under [8], this should cause the variance to increase, despite the number of 494 measurements used in its estimation increasing, until the point at which there are enough 495 repeats to capture the effects the range of scales of variation in turbulence impacting the 496 measurement. Then, this variance will become stable. At this stage we can consider the 
497 number of repeats as the minimum number required to have a robust estimation of 498 secondary velocity vectors that is to have reached estimates of velocity that are 499 asymptotic on this stable state.

500 Here we apply both methods A and B to the survey of 16 repeats at cross-section 9 in 501 Figure $3 a$ at the Lizerne-Rhône confluence. To allow a reasonable comparison, three 502 mesh cells in the middle of the cross section, and at three different depths (near the 503 surface, middle depth and near the bed) have been chosen (Figure 11). Results show

504 that by using $\underline{M}$ method $\mathrm{A}$, after six repeats, a stable variance of the velocity estimator is 505 obtained at the Lizerne-Rhône confluence (Figure 11a). Many more repeats are needed $\$ 06$ using Mmethod B (Figure 11b) and this is likely because Method B uses fewer 507 measurements per mesh cell. These results also show a higher standard deviation of the 508 velocity estimation near the surface, using Mmethod $A$ and before achieving the stable 509 situation. This can be explained by the fact that near the surface Mmethod A is more 510 sensitive to errors caused by positioning, while near the bed, hence with distance from $\$ 11$ the sounder, as the beam spread increases, the improvement obtained using Mmethod $A$ 512 is more pronounced (Figure 11a).

513 "Figure 10"

514 "Figure 11"

\section{DGPS and tilt sensor uncertainty analysis}

516 As explained above a normally distributed random error has been applied 100 times to 517 both dGPS positioning (by adding a random offset) and tilt sensors (by changing pitch and $\$ 18$ roll angles randomly) and the secondary velocities have been estimated using Mmethod 519 A for each perturbed dataset. As Figure 12 shows, the magnitude of errors related to 
520 dGPS accuracy are higher than those related to tilt sensor accuracy, for both confluences.

521 These values can reach $\pm 0.03 \mathrm{~ms}^{-1}$ and confirms the earlier finding of Rennie and Rainville 522 (2006) which showed that GPS corrections can have average errors of about $\pm 0.03 \mathrm{~ms}^{-1}$

523 (Figures 12a and 12c). These magnitudes are also higher near the surface and near the 524 bed for the Lizerne-Rhône confluence (Figure 12a). Near the surface, as there is a greater 525 random error due to ship movementsfewer measurements that can contribute to the 526 estimation of aDcp position and tilt, uncertainties in dGPS data will have a targer-greater 527 effect-on a bad velocity estimation. Near the bed, as the velocity gradient is higher, errors 528 will be greater as well. Figure $12 \mathrm{c}$ shows higher magnitudes near the surface at cross529 section 3 in Figure 3b for the Grande Eau-Rhône confluence.

530 Errors related to tilt sensor uncertainty are higher where there is a higher velocity gradient.

531 This is related to the fact that within the mesh cells with higher velocity gradients, as the 532 velocity distribution is not linear, and as averaging is made in the middle of the mesh cell, 533 it is more probable that the velocity will be affected by sensor inaccuracies of bin 534 positioning, and so be in error (Figures $12 b$ and $12 d$ ).

535 "Figure 12"

\section{Homogeneity assumption analysis}

537 Figure 13 shows the maximum inhomogeneity allowance, using Mmethod B for both case 538 studies. These results are obtained by dividing the velocity gradient obtained from 539 equation 22 by the divergence of the beams from equation 12 . They confirm that, for the $\$ 40$ homogeneity assumption to be valid and thus error to be minimized using Mmethod $\mathrm{B}$, the 541 maximum emesh cell size, which can be used is as small as $5 \mathrm{~cm}$ near the bed. Clearly, 
542 this is impossible as the configuration of the beams using aDcps always results in beam

543 divergence greater than $5 \mathrm{~cm}$

544 "Figure 13"

545 Primary and secondary flow patterns

546 In this section, we compared estimated primary and secondary velocities using methods

$547 \quad$ A and B for other cross sections in Figure 3 for both river confluences.

548 Figure 14 shows the results for cross sections 4,5 and 7 (in Figure 3a) at the Lizerne549 Rhône confluence. These cross sections also show similar results in primary and 550 secondary velocity patterns for both methods A and B. Figure 15 shows different patterns 551 in primary and secondary velocities estimation using Mmethod $A$ and $B$ for cross sections 5524,6 and 8 in Figure 3b at the Grande Eau-Rhône confluence. Method A produces-leads 553 to the identification of a stronger and more coherent tributary penetration at cross-section 5544 and weaker upwelling mid-channel, giving the impression of less intense secondary 555 circulation (Figure 15). At section 6, flow towards the true left across the shallow top of 556 the tributary point-mouth bar top-is maintained-identified and is coherent with Mmethod A.

557 At the channel-scale there is general flow convergence reflecting channel narrowing 558 (Figure 15). When usingith Mmethod $B$, these patterns are less coherent and flow is 559 towards the true right in the vicinity of the point-tributary mouth bar. These patterns are 560 repeated for section 8 (Figure 15).

561 "Figure 14"

562 "Figure 15" 


\section{Discussion}

564 In this paper we used data collected with boat-mounted aDcp technology at two 565 confluences of the Swiss river Rhône, both with similar and very low momentum ratios $566(0.018,0.022)$ and analysed these using two different methods, $A$ and $B$, to estimate 567 Cartesian velocity components. Method A is based on a methodological approach 568 developed by Vermeulen et al. (2014b). It differs by treating explicitly each individual beam 569 velocity based on its position within a predefined mesh. Results show that this method 570 reduces the volume over which the flow must be assumed to be homogenous (Fig 13). It 571 can, but not necessarily does, result in differences in estimated primary and secondary 572 velocities as compared with the more traditional method ( $B$ in this study), that involves 573 determining velocities by averaging data from the spreading beams. Our results show that 574 these differences are more pronounced in estimated secondary velocities than primary 575 velocities and are higher where there is a greater lateral velocity gradient (Figure 9). The 576 comparison between the two case studies shows that even though both confluences have 577 a very low momentum ratio, as the confluence of the Grande Eau-Rhône has a more 578 intense-complex lateral-shear zone, likely due to the effects of bed discordance, and there 579 are more significant differences in the estimation of primary and secondary velocities. This 580 is related to the extent to which spreading of the aDcp measurement beams influences 581 the secondary velocities, particularly in relation to lateral gradients in flow conditions. More 582 standard methods (Mmethod B in this study) are valid if the flow is completely 583 homogenous over the diameter of the fluid column that the beams spread. This diameter 584 varies over depth and is largest near the bed. In the case of the Grande Eau-Rhône 585 confluence where stronger lateral velocity gradients exist in the flow, individual beams will 
586 not be measuring homogenous conditions, particularly near the bed and in the zone of

587 high shear near the inner bank, because the spread of the beams may be greater in $\$ 88$ diameter than the width of the zone of lateral velocity variation. In this case, as Mmethod 589 A involves less spatial-averaging than Mmethod B, it may can-provide more accurate 590 information on the flow behavior, but such a conclusion really needs a third and 591 independent method to confirm it. At the Lizerne-Rhône confluence, even though the 592 momentum ratio is similar to Grande Eau-Rhône confluence, there is only more localized 593 tateral-shear in the flow and a simplified shear zone-; (Figure 9). In such a situation,-and 594 using Mmethod B to detect the large scale patterns of secondary flow may be more 595 advantageous, because it involves more spatial averaging.

596 The above discussion suggests that whether or not high rates of later shear influence the 597 need to adoptpotential importance of Method A depends on distance from the aDcp: with 598 more divergence at greater depths, lower levels of lateral shear are likely to be acceptable. 599 Figures $16 \mathrm{a}$ and $16 \mathrm{~b}$ quantifies the relationship between lateral velocity gradient, depth 600 and the magnitude of the relative differences in secondary velocities estimated using 601 methods $A$ and $B$ for the cross-section 6 of the Lizerne-Rhône and cross-section 3 of the 602 Grande Eau-Rhône confluences, respectively. At the Lizerne-Rhône confluence, as the 603 zone of high lateral shear is absent, even though there is a strong relationship between 604 the magnitude of the relative differences in secondary velocities estimated using methods $605 \mathrm{~A}$ and $\mathrm{B}$ and the depth (Figure 16a), their relationship with the lateral velocity gradients is 606 poor. In contrast, not as clear asfor the case of the Grande Eau-Rhône confluence (Figure 607 16b), where increasing the lateral velocity gradient and depth results in higher relative 608 differences in secondary velocities. Thus, the need to use Mmethod A will depend on the 
609 case being used and the extent to which there is lateral shear at greater distances from $\$ 10$ the aDcp. This is why whilst it may be tempting to introduce some kind of shear or velocity 611 gradient threshold to identify when Method A might be preferable, to do so would be 612 misleading as the threshold will also depend on the distance of the shear from the aDcp. 613 "Figure 16"

614 Results also confirm that several repeat transects are indispensable to provide a robust 615 estimation of secondary circulation and to reduce the effect of spatial inhomogeneity and $\$ 16$ temporal variations. Although $\underline{M}$ method $A$ reduces the minimum number of repeat 617 transects needed to estimate the secondary velocities, a larger number of these minimum 618 repeat transects (6 or more repeats for Lizerne-Rhône confluence) appeared to be $\$ 19$ required. This is higher than in the, compared to earlier findings of Szupiany et al. (2007) 620 and Vermeulen et al., (2014b) who argue that 5 repeats are enough to have a robust $\$ 21$ estimation of the turbulence averaged velocity. We also note that an even number of 622 repeats may be important to avoid directional bias in dGPS positions.

623 As aDep data obtained from multiple transects are notoriously noisy, another approach to 624 averaging involves post-processing that takes binned data estimated from multiple 625 transects, and averaging these data through spatial smoothing. This is adopted in the 626 Velocity Mapping Toolbox (VMT) (Parsons et al., 2013). The VMT maps ensembles onto 627 the mean straight cross-section and interpolates each one of these grid nodes using linear 628 interpolation. The bed profile is estimated using the mean of the four beams. These 629 projected and interpolated velocity data from each set of transects are averaged using a 630 simple arithmetic averaging, at every grid node, to provide a composite representation of 631 the velocity field. Once the averaging is complete for all the nodes, a coordinate 
632 transformation is applied to transform Earth velocity components intovelocity components 633 in the plane of the cross section (U, V and W) (Parsons et al., 2013). The VMT can also 634 use a smoothing window which is a moving average and it averages every velocity vector 635 with its nearest neighbor. The user can define the horizontal and vertical smoothing 636 window size.

637 It was not the aim of this paper to evaluate the specific VMT method, but to put our 638 comparison of Methods $A$ and B into context, Figure 17 a shows results obtained for 639 primary and secondary velocities for the VMT, at cross section 6 in Figure $3 a$ at the 640 Lizerne-Rhône confluence, that is comparable with Figures $4 a$ and $4 \mathrm{~b}$ for our Methods $\mathrm{A}$ 641 and B. The pattern of primary and secondary velocities are similar to each other for all 642 three methods (Figures 17a, 4a and 4b). Although the VMT results have been obtained 643 using a horizontal and vertical smoothing window sizes of 2 , they are not as coherent as 644 the results obtained using methods $A$ and $B$, suggesting that using the VMT requires more 645 repeat transects or more repeat stationary measurements.

646 Figure $17 \mathrm{~b}$ shows primary and secondary velocities estimated using the VMT for cross647 section 3 in Figure $3 b$ at the Grande Eau-Rhône confluence. Again, as the VMT uses a 648 straight mean cross section, estimated velocities are not as coherent as those of method 649 A and B (Figures $4 c$ and $4 d$ ).

650 "Figure 17"

651 Since Mmethod $A$ is based on the position of beams, if the bin position errors related to 652 dDGPS accuracy as well as sensor tilt are greater than homogeneity errors associated 653 with beam divergence, standard $\underline{M}$ method $B$ is more reliable. This is likely to be the case 
654 particularly the case-in rivers smaller-shallower than those studied here rivers-and where 655 high resolution is required due to large velocity gradients. In big-rivers of the scale studied 656 here, and deeper, by increasing the mesh cell size, we can still have sufficient data to 657 estimate velocity vectors, and the errors related toeffects dGPS and tilt sensor errors have 658 a minor effect._-This confirms the earlier findings by Vermeulen et al., (2014b), which 659 showed that Mmethod A provides the greatest improvement where the aDcp cell size is 660 much smaller than the beam spread. We are not yet in a position to identify the depth at 661 which Method A becomes preferable to Method B, and again this will depend on other 662 parameters such as the intensity of shear and so may not be readily generalizable 663 between confluences.

664 The difficulty of identifying the depths of rivers and intensities of shear that make one 665 method preferable over another precludes adoption of simple quantitative guidance on 666 which method to use when. As both methods have some disadvantages, we argue that 667 both methods should be applied. If they give similar results, then there should be 668 confidence in both. If and where they differ, analysis should be undertaken to identify why, 669 and hence which method is likely to be preferable. Association of the differences in 670 primary and secondary velocities inferred between the two methods with estimates of 671 shear intensity and with estimated tilt and positioning errors should then help decide 672 whether Method A or Method B is preferable in a particular case. This preference may 673 vary between confluences but also through time at a confluence, if shear or flow depth 674 changes significantly between survey dates.

675 Finally, we wish to emphasise that the impact of averaging is only one element that must 676 be considered in obtaining reliable primary and secondary clow estimates in river 
677 confluences. Other issues, such as the rotation method needed to distinguish primary and
678 secondary circulation, remain important and should be considered routinely.

679 Conclusions

680 This paper shows the advantage of working with the radial (beam) velocity measurements 681 of an aDcp within each bin prior to averaging them across a given volume of fluid 682 (Mmethod A) as opposed to identify volumes of fluid and assuming bend homogeneity 683 within them (Mmethod B). Such a treatment is important where there are strong velocity 684 gradients in the flow as with river channel confluences. In the first of our case-study 685 confluences, the Lizerne-Rhône, a very small tributary joined the main river, and the 686 pattern of primary and secondary velocities obtained with methods $A$ and $B$ were relatively 687 similar, more so for primary velocities. But for a second confluence, the Grande Eau688 Rhône, with a similar momentum ratio, there were much larger differences. We attributed 689 this to the formation of much stronger shear at this confluence. Method A also appeared 690 to reduce the number of repeat transects needed to estimate secondary velocities reliably.

691 The main downside is that $\underline{M}$ method $A$ is more sensitive to errors related to positioning. 692 Thus, good dGPS accuracy and precision are required to perform a robust estimation of 693 velocity.

694 In smaller/shallower rivers, Method B may be acceptable indeed preferable as it is less 695 sensitive to GPS errors. In larger rivers, Method A may be necessary, especially in the 696 presence of strong shear at the confluence. Choice between these mAethods should be 697 based upon an initial screening of the extent to which there is strong shear in the flow as 698 well as the extent to which bins further from the aDcp are influenced by beam divergence. 


\section{Appendix A}

700 The LOWESS model is a locally weighted polynomial regression, which at each point and 701 in the range of dataset, a low degree polynomial is fitted to a subset of the data, using 702 weighted least squares. This polynomial fit gives more weight to points closer to the point 703 whose response is being estimated. The value of the regression function for the point is 704 then obtained by evaluating the local polynomial using the explanatory variable values for 705 that data point. The LOWESS fit is complete after regression function values have been 706 computed for each of the $n$ data points. Many of the details of this method, such as the 707 degree of the polynomial model and the weights, are flexible ("Local regression,” n.d.).

\section{Acknowledgements}

709 This paper benefited from constructive and detailed suggestions from two anonymous 710 reviewers and Editor Mike Kirkby. The work was supported by Swiss National Science 711 Foundation Grant 200021_160020. The codes used may be obtained upon request to 712 Gelare Moradi.

\section{References}

714 Alvarez, L. V., Schmeeckle, M.W., Grams, P.E., 2017. A detached eddy simulation model 715 for the study of lateral separation zones along a large canyon-bound river. J. Geophys. 716 Res. Earth Surf. 122, 25-49. https://doi.org/10.1002/2016JF003895.

717 Ashmore, P.E., Ferguson, R.I., Prestegaard, K.L., Ashworth, P.J. and Paola, C., 1992. 718 Secondary flow in coarse-grained braided river confluences. Earth Surface Processes 719 and Landforms, 17, 299-312. 
720 Best, J.L. and Roy, A.G., 1991. Mixing-layer distortion at the confluence of channels of 721 different depth. Nature, 350, 411-413.

722 Biron, P., De Serres, B., Roy, A.G., Best, J.L., 1993. Shear layer turbulence at an unequal 723 depth channel confluence. In: Clifford, N.J., French, J.R., Hardisty J. (editors) 724 Turbulence: Perspectives on Flow and Sediment Transport, Wiley, Chichester, 197725213.

726 Dinehart, R.L. and Burau, J.R., 2005. Averaged indicators of secondary flow in repeated 727 acoustic Doppler current profiler crossings of bends. Water Resources Research, 41, $728 \quad$ W09405.

729 Engel, F.L. and Rhoads, B.L., 2016. Three-dimensional flow structure and patterns of bed 730 shear stress in an evolving compound meander bend. Earth Surface Processes and $731 \quad$ Landforms, 41, 1211-1226.

732 Gualtieri, C., Ianniruberto, M., Filizola, N., Santos, R. and Endreny, T., 2017. Hydraulic 733 complexity at a large river confluence in the Amazon basin. Ecohydrology, 10, e1863

734 Gunawan, B., Neary, V.S., McNutt, J.R., 2011. Ornl Adv Post-Processing Guide and 735 Matlab Algorithms for Mhk Site Flow and Turbulence Analysis, Ornl/Tm-2011/404. 736 doi:10.2172/1034377.

737 Jackson, P.R., Garcia, C.M., Oberg, K.A., Johnson, K.K. and Garcia, M.H., 2008. Density 738 currents in the Chicago River: Characterization, effects on water quality, and potential 739 sources. Science of the Total Environment 401: 130-143. 
740 Kasvi, E., Vaaja, M., Alho, P., Hyyppä, H., Hyyppä, J., Kaartinen, H. and Kukko, A., 2013. 741 Morphological changes on meander point bars associated with flow structure at 742 different discharges. Earth Surface Processes and Landforms, 38, 577-590.

743 Kasvi, E., Laamanen, L., Lotsari, E. and Alho, P., 2017. Flow Patterns and Morphological 744 Changes in a Sandy Meander Bend during a Flood-Spatially and Temporally Intensive 745 ADCP Measurement Approach. Water, 9, 106.

746 Knox, R. L. and Latrubesse, E.M., 2017. A geomorphic approach to the analysis of 747 bedload and bed morphology of the Lower Mississippi River near the Old River Control $748 \quad$ Structure. Geomorphology, 268, 35-47.

749 Konsoer, K.M. and Rhoads, B.L., 2014. Spatial-temporal structure of mixing interface $750 \quad$ turbulence at two large river confluences. Environmental Fluid Dynamics, 14, $1043-$ $751 \quad 1070$.

752 Kostaschuk, R.A., Shugar, D., Best, J.L., Parsons, D.R., Lane, S.N., Hardy, R.J. and 753 Orfeo, O., 2009. Suspended sediment transport and deposition over a dune: Río 754 Paraná, Argentina. Earth Surface Processes and Landforms, 34, 1605-1611.

755 Lane, S.N., Parsons, D.R., Best, J.L., Orfeo, O., Kostaschuk, R. a., Hardy, R.J., 2008. 756 Causes of rapid mixing at a junction of two large rivers: Río Paraná and Río Paraguay, 757 Argentina. Journal of Geophysical Research, 113, 1-16. $758 \quad$ https://doi.org/10.1029/2006JF000745.

759 Lane, S.N., Bradbrook, K.F., Richards, K.S., Biron, P.M., Roy, a. G., 2000. Secondary 760 circulation cells in river channel confluences : measurement artefacts or coherent flow 
761 structures? Hydrological Processes, 14, 2047-2071. https://doi.org/10.1002/1099762 1085(20000815/30)14:11/12<2047::aid-hyp54>3.0.co;2-4.

763 Local regression [WWW Document], n.d.

764 URL:https://en.wikipedia.org/wiki/Local_regression (accessed 1.22.18).

765 Lotsari, E., Kasvi, E., Kämäri, M., and Alho, P., 2017. The effects of ice cover on flow 766 characteristics in a subarctic meandering river. Earth Surface Processes and 767 Landforms, 42, 1195-1212.

768 Marsden, R.F. and Ingram, R.G., 2004. Correcting for beam spread in acoustic Doppler 769 current profiler measurements. Journal of Atmospheric and Oceanice Technology, 21, $770 \quad 1491-1498$.

771 Morlock, S.E., 1996. Evaluation of Acoustic Doppler Current Profiler Measurements of 772 River Discharge. U.S. Geological Survey Water Resources Investigations Report 957734218.

774 Muste, M., Yu, K. and Spasojevic M. 2004. Practical aspects of ADCP data use for 775 quantification of mean river flow characteristics: Part I: Moving-vessel measurements. $776 \quad$ Flow Measurement and Instrumentation, 15, 1-16.

777 Parsapour-Moghaddam, P. and Rennie, C.D., 2018. Calibration of a 3D Hydrodynamic 778 meandering river model using fully spatially distributed 3D ADCP velocity data. Journal 779 of Hydraulic Engineering, 144, 04018010.

780 Parsons, D.R., Best, J.L., Orfeo, O., Hardy, R.J., Kostaschuk, R.A. and Lane, S.N., 2005. 781 The morphology and flow fields of three-dimensional dunes, Rio Paraná, Argentina: 
782 results from simultaneous multibeam echo sounding and acoustic Doppler current 783 profiling. Journal of Geophysical Research, 110, F04S03.

784 Parsons, D.R., Best, J.L., Lane, S.N, Orfeo, O., Hardy, R.J. and Kostaschuk, R.A., 2007. 785 Form roughness and the absence of secondary flow in a large confluence-diffluence, 786 Rio Paraná, Argentina. Earth Surface Processes and Landforms, 32, 155-162.

787 Parsons, D.R., Jackson, P.R., Czuba, J.A., Engel, F.L., Rhoads, B.L., Oberg, K.A., Best, 788 J.L., Mueller, D.S., Johnson, K.K., Riley, J.D., 2013. Velocity Mapping Toolbox (VMT): 789 A processing and visualization suite for moving-vessel ADCP measurements. Earth 790 Surface Processes and Landforms, 38, 1244-1260.

791 Petrie, J., Diplas, P., Gutierrez, M. and Nam, S., 2013. Combining fixed- and moving792 vessel acoustic Doppler current profiler measurements for improved characterization 793 of the mean flow in a natural river. Water Resources Research, 49, 5600-5614.

794 Rennie, C.D., Millar, R.G. and Church, M.A., 2002. Measurement of bed load velocity 795 using an acoustic Doppler current profiler. Journal of Hydraulic Engineering - ASCE, $796 \quad 128,473-483$.

797 Rennie, C.D. and Millar, R.G., 2004. Measurement of the spatial distribution of fluvial 798 bedload transport velocity in both sand and gravel. Earth Surface Processes and 799 Landforms, 29, 1173-1193.

800 Rennie, C.D., Rainville, F., 2006. Case study of precision of GPS differential correction 801 strategies: influence on aDcp Velocity and discharge estimates. Journal of Hydraulic $802 \quad$ Engineering, 132, 225-234. 
803 Rennie, C. D., and Church, M., 2010. Mapping spatial distributions and uncertainty of 804 water and sediment flux in a large gravel bed river reach using an acoustic Doppler 805 current profiler, Journal of Geophysical Research - Earth Surface, 115.

806 Rhoads, B.L. and Kenworthy, S.T., 1995. Field measurements of flow structure at a high807 angle asymmetrical stream confluence. Geomorphology 11, 273-293.

808 Rhoads, B.L. and Kenworthy, S.T., 1998. Time-averaged flow structure in the central 809 region of a stream confluence. Earth Surface Processes and Landforms, 23, 171-191.

810 Rhoads, B.L., and Sukhodolov, A.N., 2001. Field investigation of three-dimensional flow 811 structure at stream confluences: 1 . Thermal mixing and time-averaged velocities. Water 812 Resources Research, 27, 2393-410.

813 Rhoads, B.L., and Sukhodolov, A.N., 2004. Spatial and temporal structure of shear layer 814 turbulence at a stream confluence. Water Resources Research, 40, W06304.

815 Rhoads, B.L., and Sukhodolov, A.N., 2008. Lateral momentum flux and the spatial 816 evolution of flow within a confluence mixing interface. Water Resources Research, 44, $817 \quad$ W08440.

818 Riley, J.D. and Rhoads, B.L., 2012. Flow structure and channel morphology at a natural 819 confluent meander bend. Geomorphology, 163, 84-98.

820 Riley, J.D., Rhoads, B.L., Parsons, D.R. and Johnson K.K., 2015. Influence of junction 821 angle on three-dimensional flow structure and bed morphology at confluent meander 822 bends during different hydrological conditions. Earth Surface Processes and 823 Landforms, 40, 252-271. 
824 Shugar, D.H., Kostaschuk, R., Best, J.L., Parsons, D.R., Lane, S.N., Orfeo, O. and Hardy, 825 R,J., 2010. On the relationship between flow and suspended sediment transport over 826 the crest of a sand dune, Río Paraná, Argentina. Sedimentology 57: 252-272.

827 SonTek YSI, 2000. Acoustic Doppler Profiler (ADP ®) Principles of Operation.

828 SonTek YSI, 2010. RiverSurveyor S5/M9 System Manual 115.

829 Sukhodolov, A.N. and Rhoads, B. L., 2001. Field investigation of three-dimensional flow 830 structure at stream confluences 2. Turbulence. Water Resources Research, 27, 24118312424.

832 Sukhodolov, A. N., Krick, J., Sukhodolova, T. A., Cheng, Z., Rhoads, B. L. and 833 Constantinescu, G. S., 2017. Turbulent flow structure at a discordant river confluence: 834 Asymmetric jet dynamics with implications for channel morphology. Journal of 835 Geophysical Research: Earth Surface, 122(6), 1278-1293. 836 https://doi.org/10.1002/2016JF004126.

837 Szupiany, R.N., Amsler, M.L., Best, J.L. and Parsons, D.R., 2007. Comparison of fixed838 and moving vessel measurements with an aDp in a large river. Journal of Hydraulic 839 Engineering, 133, 1299-1309.

840 Szupiany, R.N., Amsler, M.L., Parsons, D.R. and Best, J.,L., 2009. Morphology, flow 841 structure, and suspended bed sediment transport at two large braid-bar confluences. $842 \quad$ Water Resources Research, 45, W05415.

843 Szupiany, R.N., Amsler, M.L., Hernandez, J., Parsons, D.R., Best, J.L., Fornari, E. and 844 Trento, A., 2012. Flow fields, bed shear stresses, and suspended bed sediment 845 dynamics in bifurcations of a large river. Water Resources Research, 48, W11515. 
846 Tomas, G., Bleninger, T., Rennie, C.D., Guarneri, H., 2018. Advanced 3D mapping of 847 hydrodynamic parameters for the analysis of complex flow Motions in a submerged 848 bedrock canyon of the Tocantins River, Brazil. Water (Switzerland) 10, 1-19. 849 https://doi.org/10.3390/w10040367.

850 Tsubaki, R., Kawahara, Y., Muto, Y. and Fujita, I., 2012. New 3-D flow interpolation 851 method on moving ADCP data. Water Resources Research, 48, 1-15.

852 Venditti, J.G., Rennie, C.D., Bomhof, J., Bradley, R.W., Little, M., Church, M., 2014. Flow 853 in bedrock canyons. Nature 513, 534-537. https://doi.org/10.1038/nature13779.

854 Vermeulen, B., Hoitink, A.J.F., van Berkum, S.W. and Hidayat, 2014a. Sharp bends 855 associated with deep scours in a tropical river: The river Mahakam (East Kalimantan, 856 Indonesia). Journal of Geophysical Research, Earth Surface, 119, 1441-1454.

857 Vermeulen, B., Sassi, M.G. and Hoitink, A.J.F., 2014b. Improved flow velocity estimates 858 from moving-boat ADCP measurements. Water Resources Research, 50, 4186-4196.

859 Vermeulen, B., Hoitink, A.J.F. and Labeur, R.J., 2015. Flow structure caused by a local 860 cross-sectional area increase and curvature in a sharp river bend. Journal of 861 Geophysical Research, Earth Surface, 120, 1771-1783.

862 Zhao, J., Chen, Z., and Zhang, H., 2014. A robust method for determining the heading 863 misalignment angle of GPS compass in ADCP measurement. Flow Measurements and 864 Instrumentation, 35, 1-10. 


\begin{tabular}{|l|c|c|}
\hline Sites & Lizerne & Grande eau \\
\hline Tributary upslope contributing area $\left(\mathrm{km}^{2}\right)$ & 64.8 & 132 \\
\hline Main stem upslope contributing area $\left(\mathrm{km}^{2}\right)$ & 3401 & 5088 \\
\hline Basin area ratio & $1.89 \%$ & $2.59 \%$ \\
\hline Tributary width (m) & 6.5 & 16.5 \\
\hline Main stem width upstream of junction $(\mathrm{m})$ & 46 & 58 \\
\hline Width ratio & 0.15 & 0.28 \\
\hline Junction angle ( $\left.{ }^{\circ}\right)$ & 80 & 70 \\
\hline Tributary Froude number & 0.32 & 0.05 \\
\hline Bed slope of the tributaries upstream of the confluence $(\%)$ & $\sim 0.5$ & $0.5-1$ \\
\hline Main stem slope upstream of the confluence $(\%)$ & 2 & 2.2 \\
\hline Tributary slope ( $\left.{ }^{\circ}\right)$ & 33.1 & 26.6 \\
\hline Rhône discharge during measurement $\left(\mathrm{m}^{3} \mathrm{~s}^{-1}\right)$ & $\underline{182}$ & $\underline{300}$ \\
\hline Tributary discharge during measurement $\left(\mathrm{m}^{3} \mathrm{~s}^{-1}\right)$ & $\underline{4}$ & $\underline{8.13}$ \\
\hline Discharge ratio during measurement & 0.018 & 0.027 \\
\hline Momentum ratio (Mr) during measurement & & 0.022 \\
\hline
\end{tabular}

865

Table 1: Selected upper Rhône tributaries with their typical characteristics

866 
Bed elevation \& Best fit

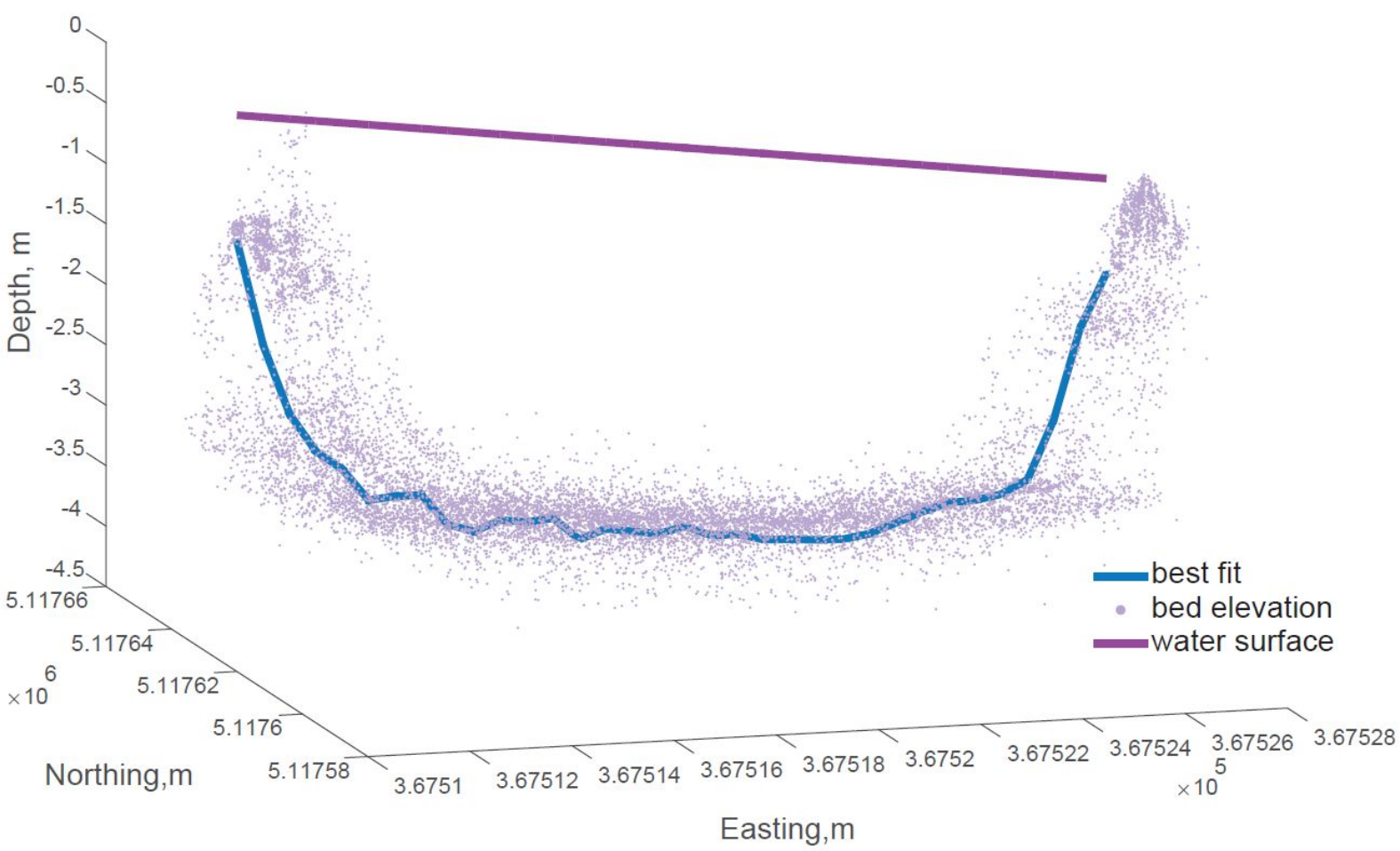

867

Easting, $m$

868

Figure 1: Bed elevations, the best fit to those elevations and the water level representation

http://mc.manuscriptcentral.com/esp 


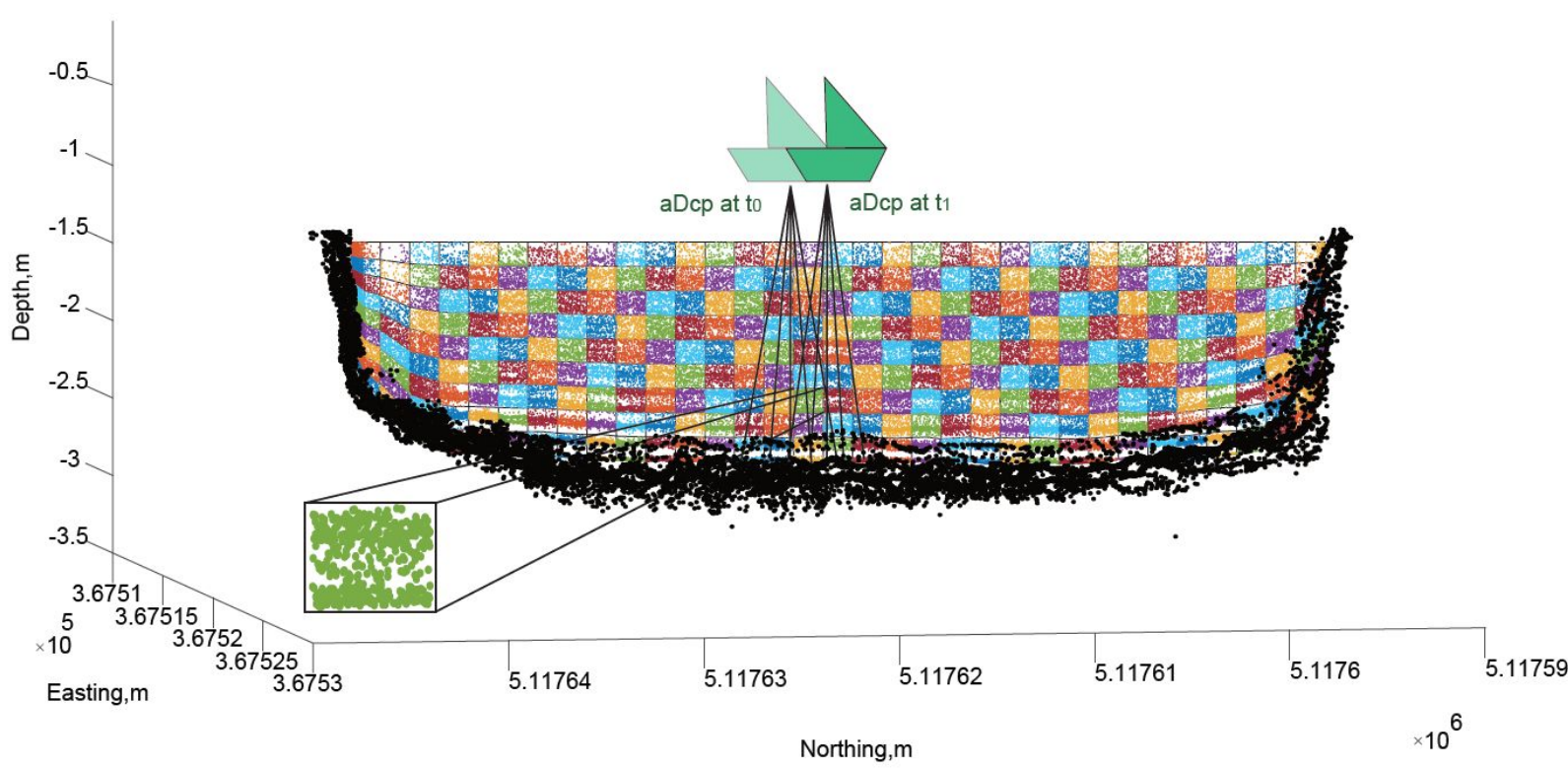

$870 \quad$ Figure 2: An example of beam velocity positioning within each mesh cell, using Mmethod A, dots show beam 871 velocities and color is an automatic Matlab function to distinguish between different mesh cells 

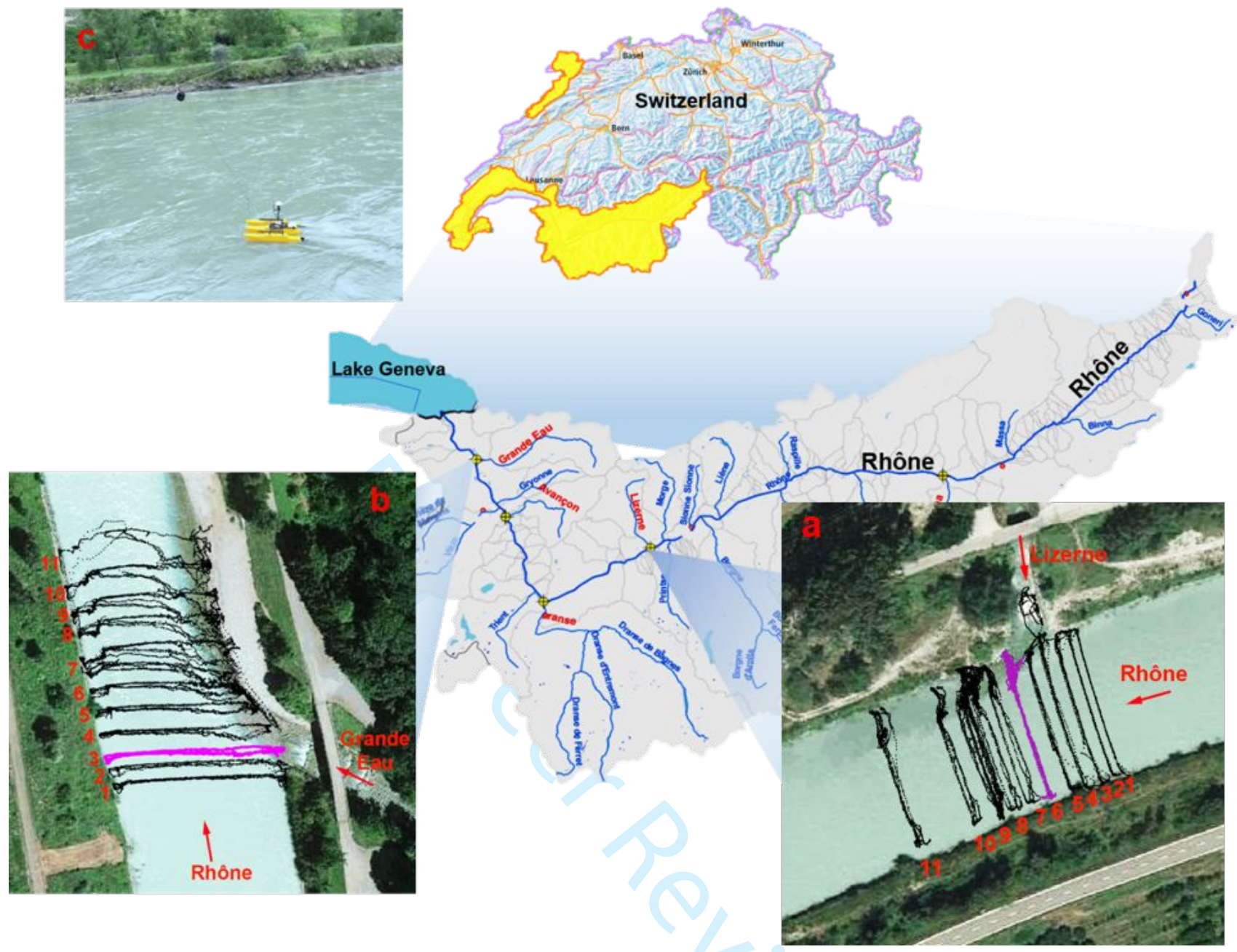

Figure 3: Tracks navigated by SonTek aDcp moving boat system at a) Lizerne-Rhône confluence near Vétroz, at 874 07/07/2017 and b) Grande Eau-Rhône confluence near Aigle at 23/05/2018. The repeated transect data assessed in 875 this paper are from cross-section 6 at the Lizerne-Rhône confluence a and cross-section 3 at the Grande Eau-Rhône 876 confluence and c) Rope-Pulley system 


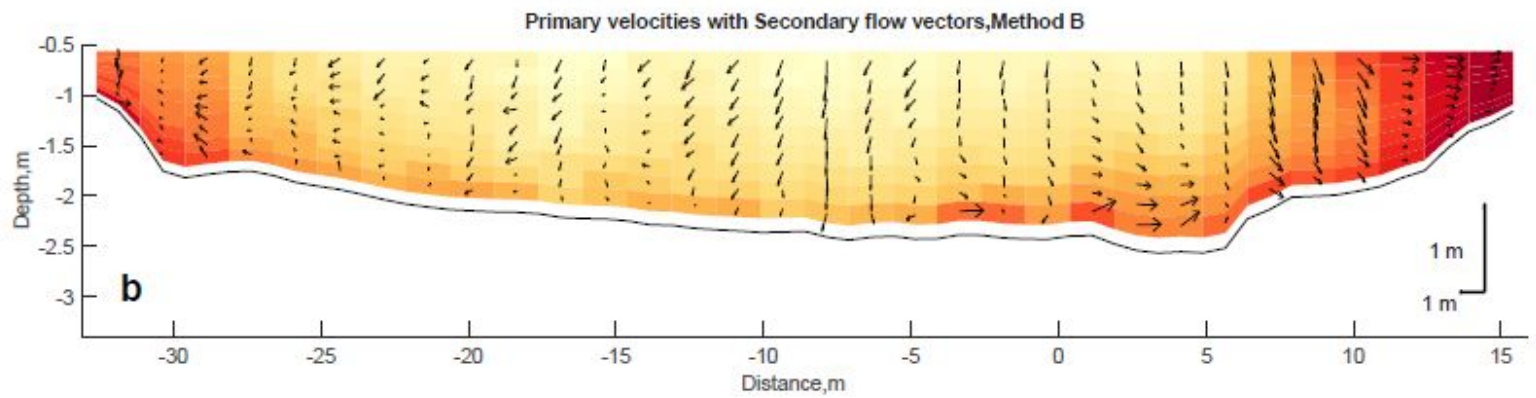

Primary velocities, $\mathrm{m} / \mathrm{s}$

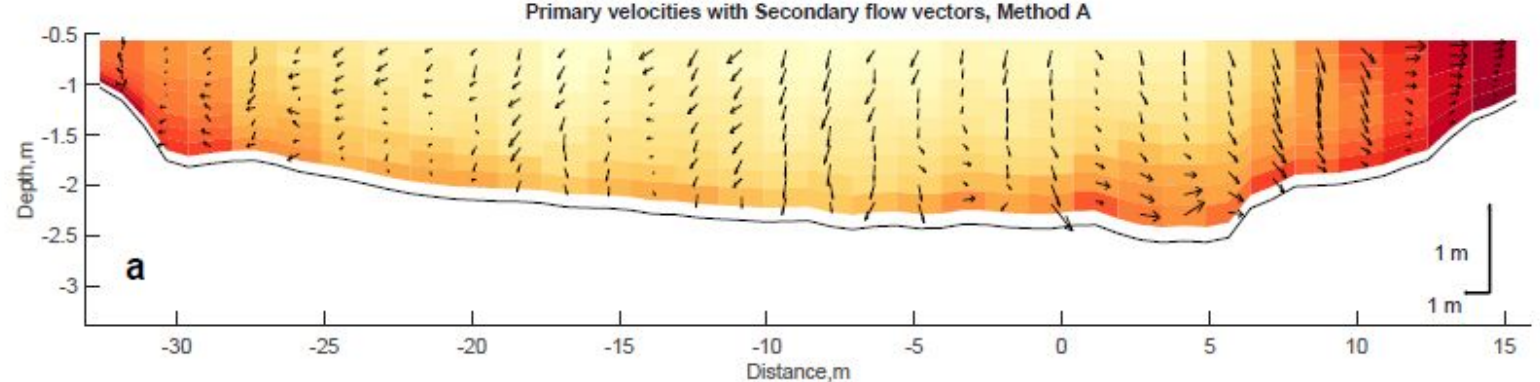

Primary velocities, $\mathrm{m} / \mathrm{s}$
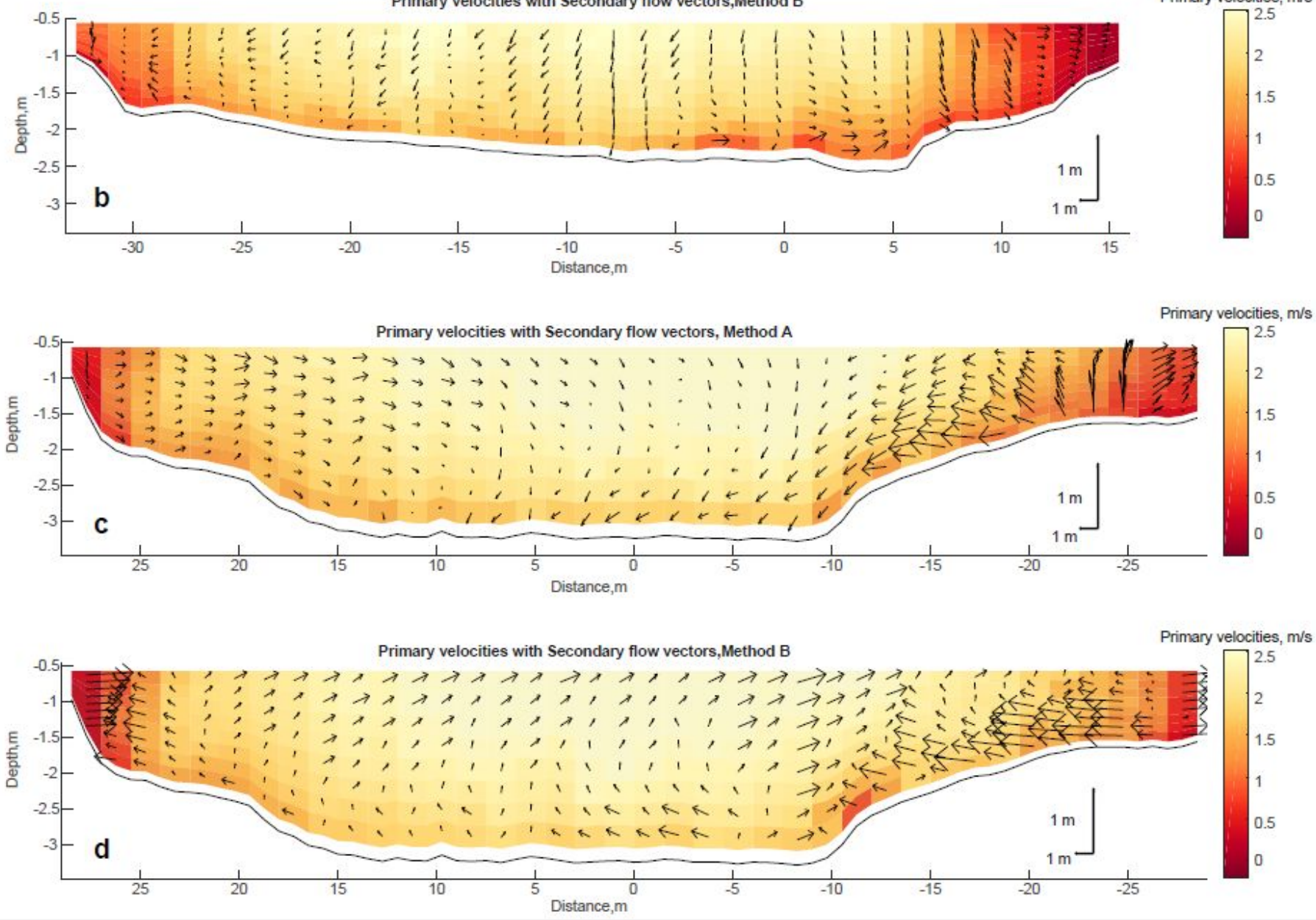

Figure 4: Primary and secondary velocities estimated with method A at a) for the Lizerne-Rhône Method A (a) and Method B (b) and the, cross-section 9 and b) Grande Eau-Rhêne, cross-section 3, confluences and method B at $G)$ Lizerne-Rhône, cross-section 9, and d) Grande Eau-Rhône Method A (c) and Method B (d); view is looking downstream., cross-section 3, confluences 

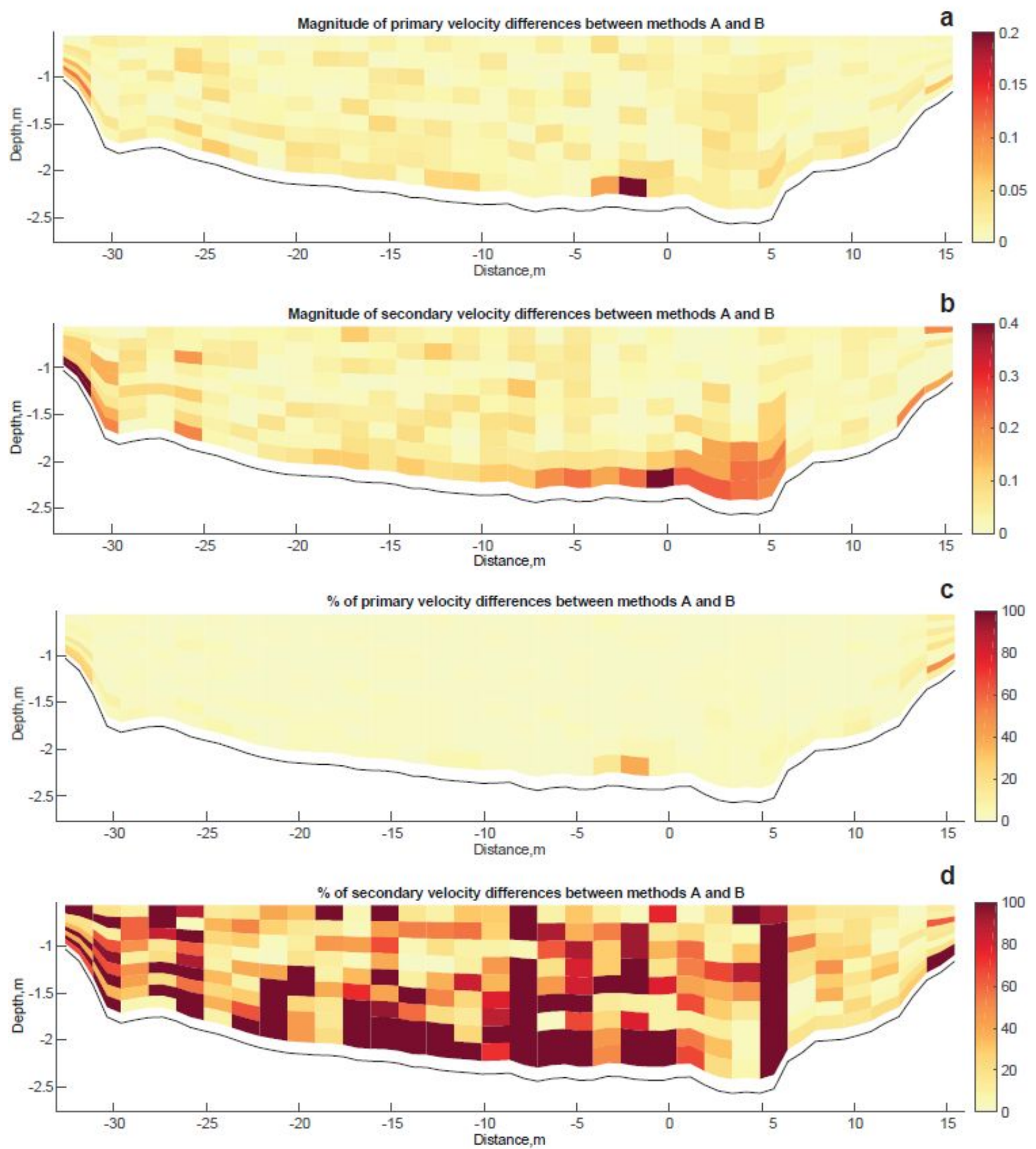

Figure 5: Differences between magnitude of primary and secondary velocities $(\mathrm{m} / \mathrm{s})$ between methods $A$ and $B$ (a and b) and the percentages of their difference $(\mathrm{m} / \mathrm{s})(\mathrm{c}$ and d), at the Lizerne-Rhône confluence, for cross-section 9; view is looking downstream. 

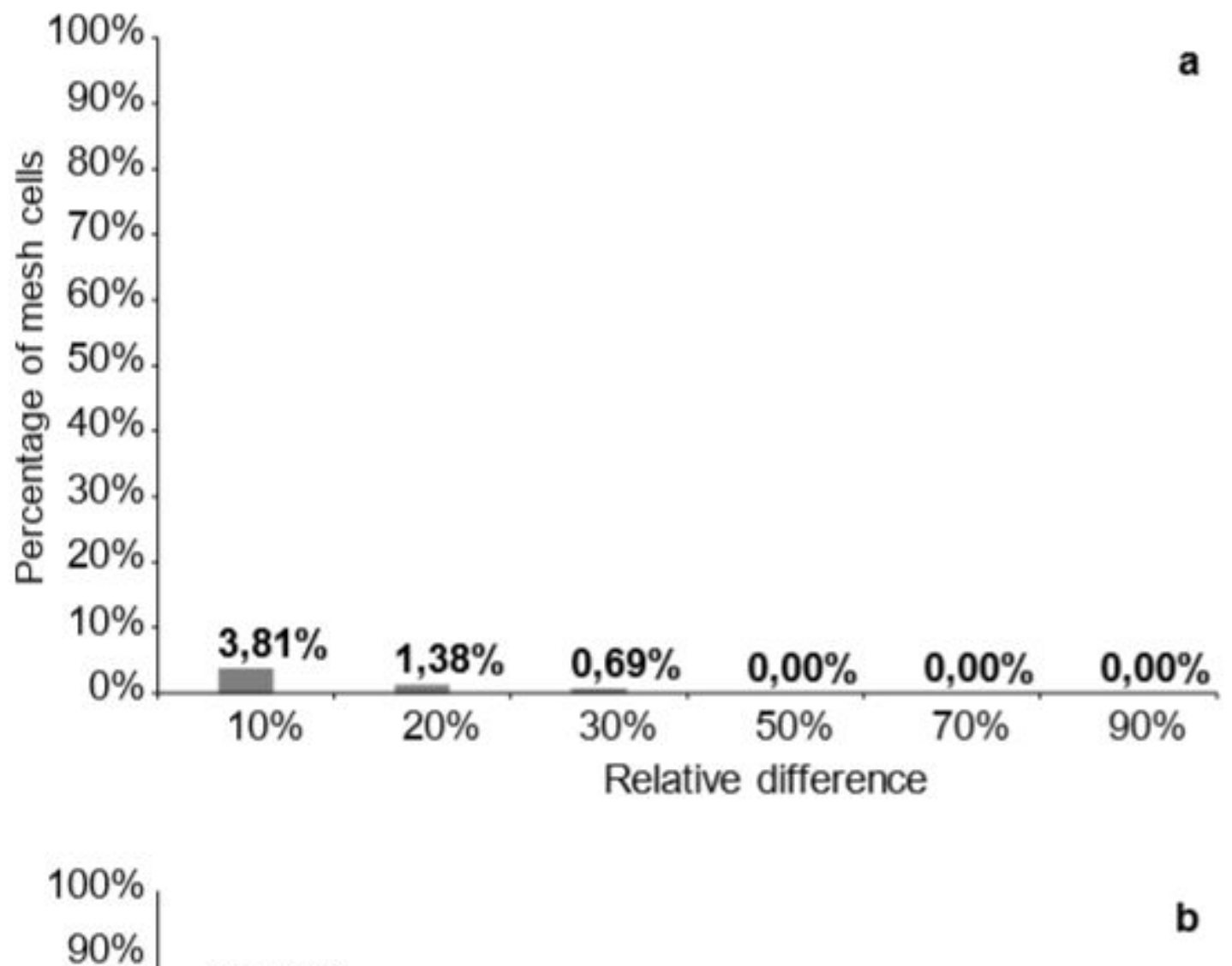

Figure 6: Relative differences in a) primary velocity magnitudes and b) secondary velocity magnitudes, between $10 \%$ $0 \%$
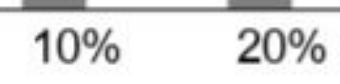

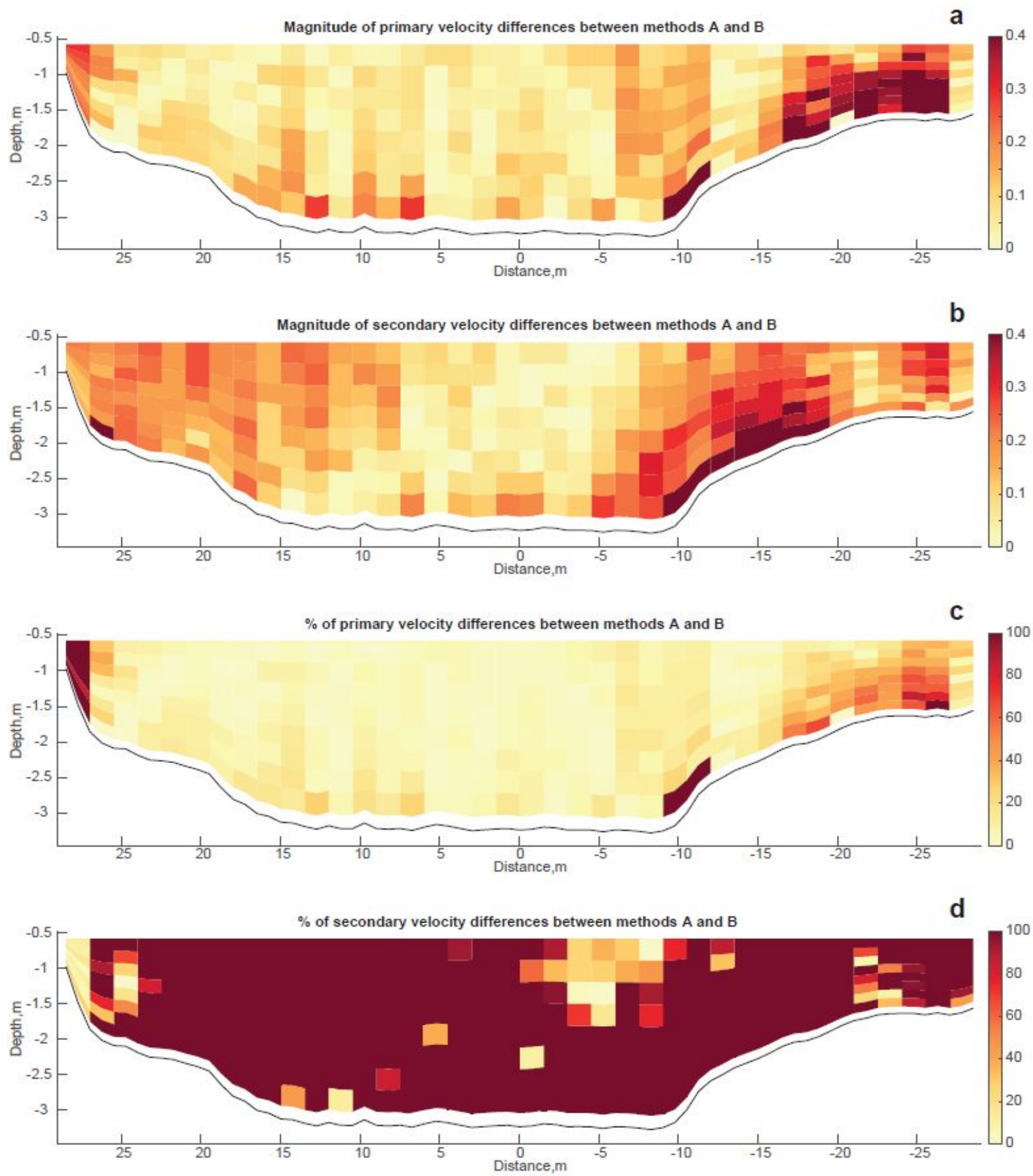

890 Figure 7: Differences between magnitude of primary and secondary velocities $(\mathrm{m} / \mathrm{s})$ between methods $A$ and $B$ (a and 891 b) and the percentages of their difference $(\mathrm{m} / \mathrm{s})$ (c and d), at the Grande Eau-Rhône confluence, for cross-section 3 ; view is looking downstream. 

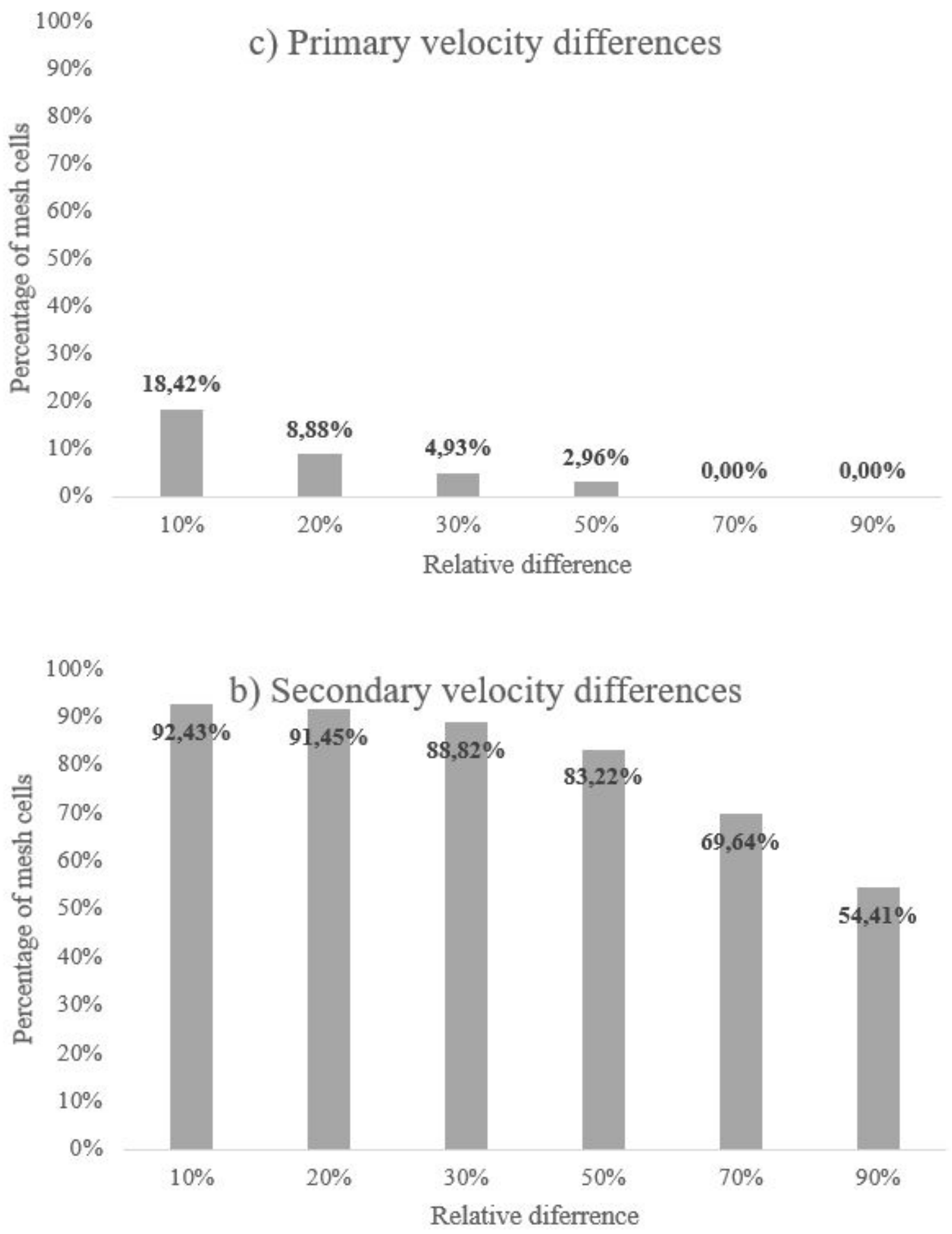

893

Figure 8: Relative differences in a) primary velocity magnitudes and b) secondary velocity magnitudes, between $894 \quad$ Figure 8. Relative differents A and B, at the Grande Eau-Rhône confluence, for cross-section 3; view is looking downstream. 

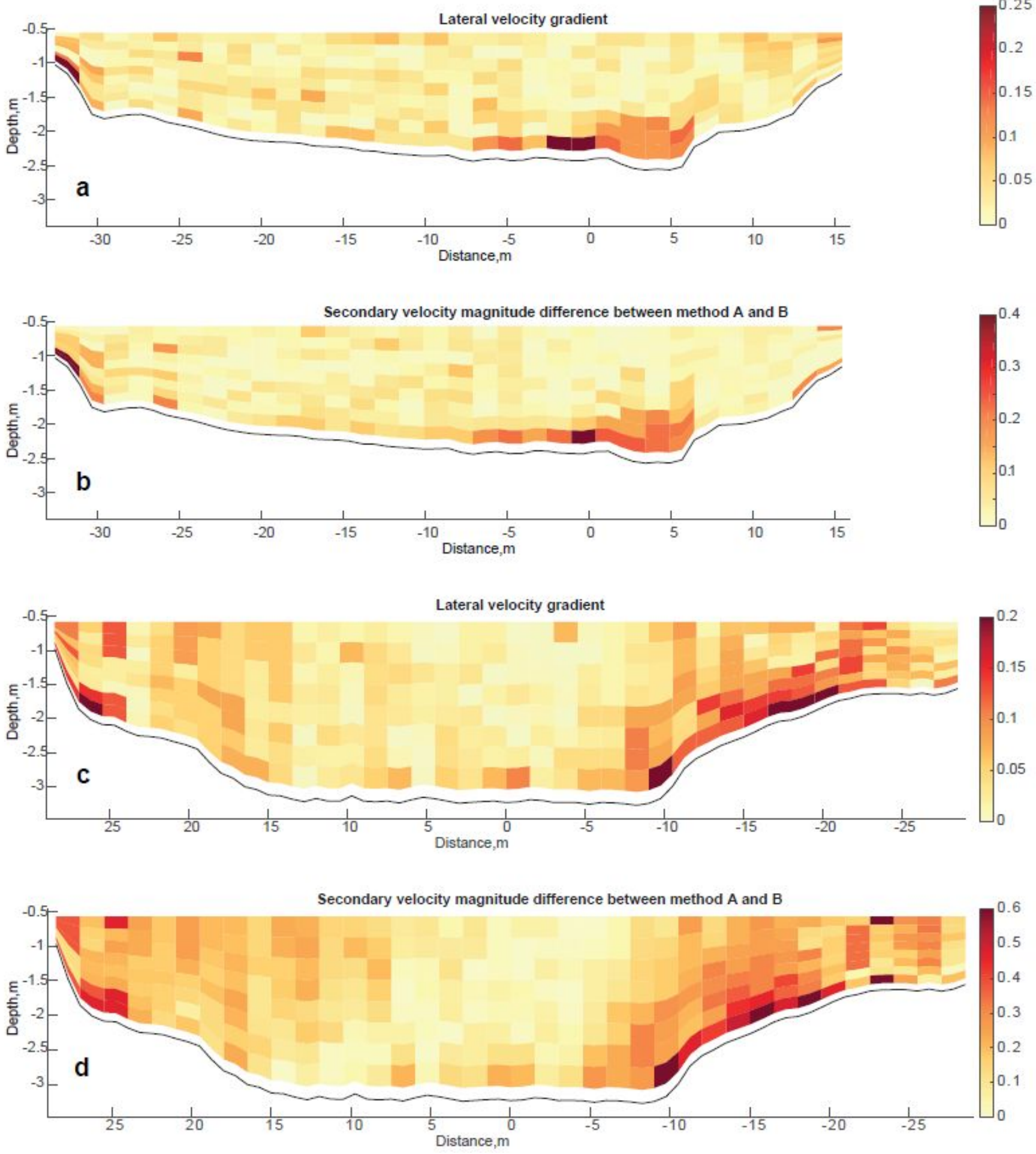

Figure 9: Lateral velocity gradients (a, c) and differences in the secondary velocity magnitudes $(b, d)$ at the LizerneRhône cross-section $9(a, b)$ and the Grande Eau-Rhône cross-section $3(c, d)$ confluences: view is looking downstream. 


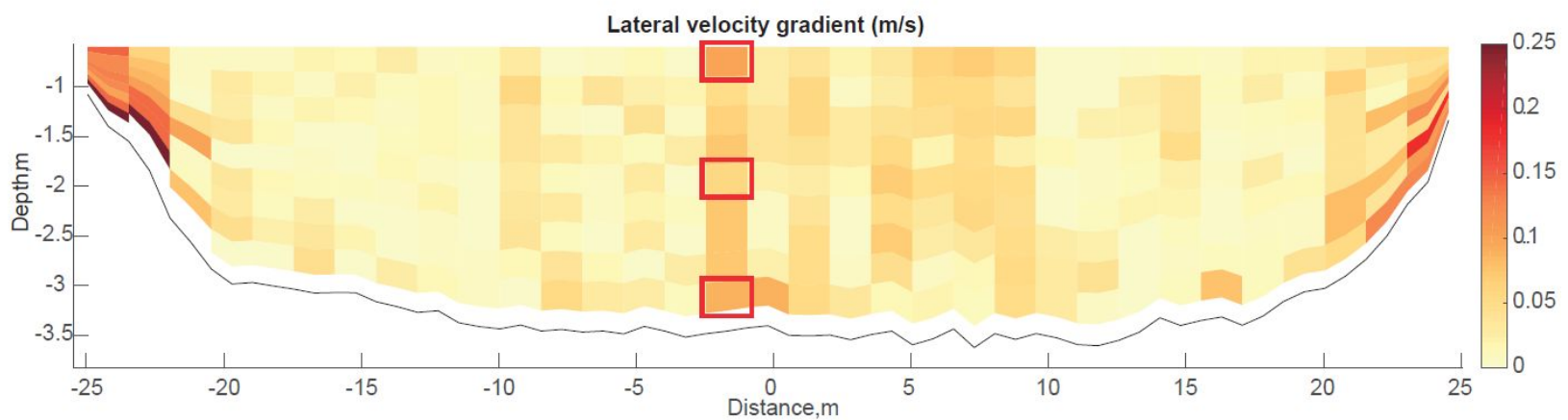

900

901 Figure 10: Water column and mesh cells for cross section 9 in Figure 3a at the Lizerne-Rhône confluence, in which standard deviation of the estimated velocities have been calculated; view is looking downstream. 

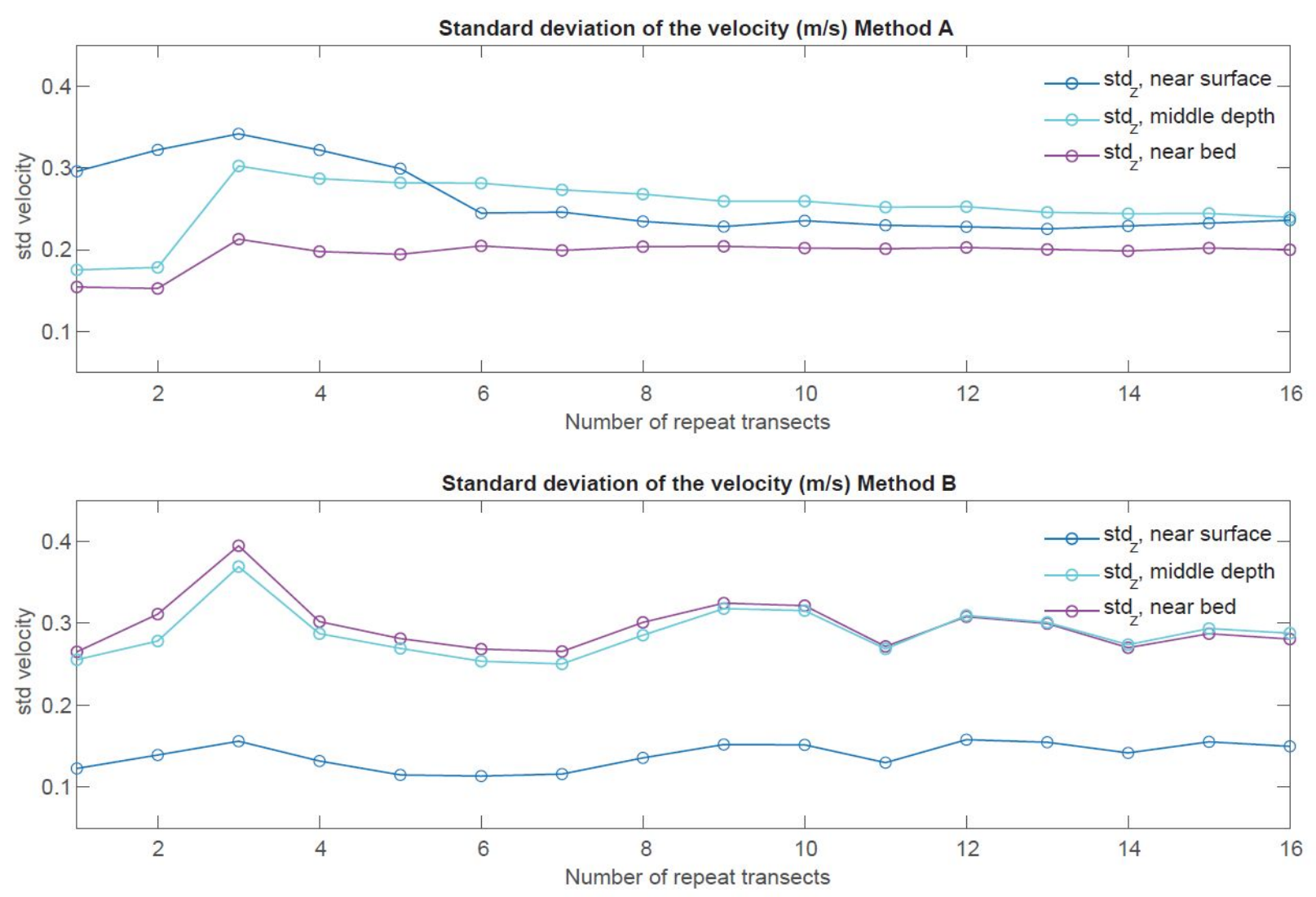

903

904 Figure 11: Standard deviation of the velocity estimated using methods A and B for 16 repeats at the Lizerne-Rhône 905 confluence cross-section 9 


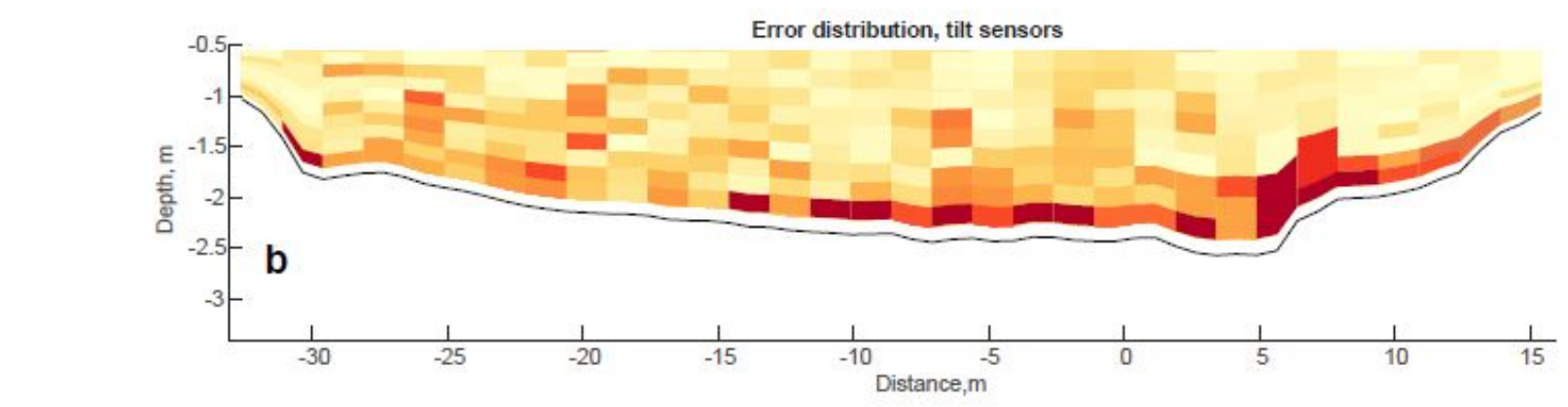

Magnitude of error

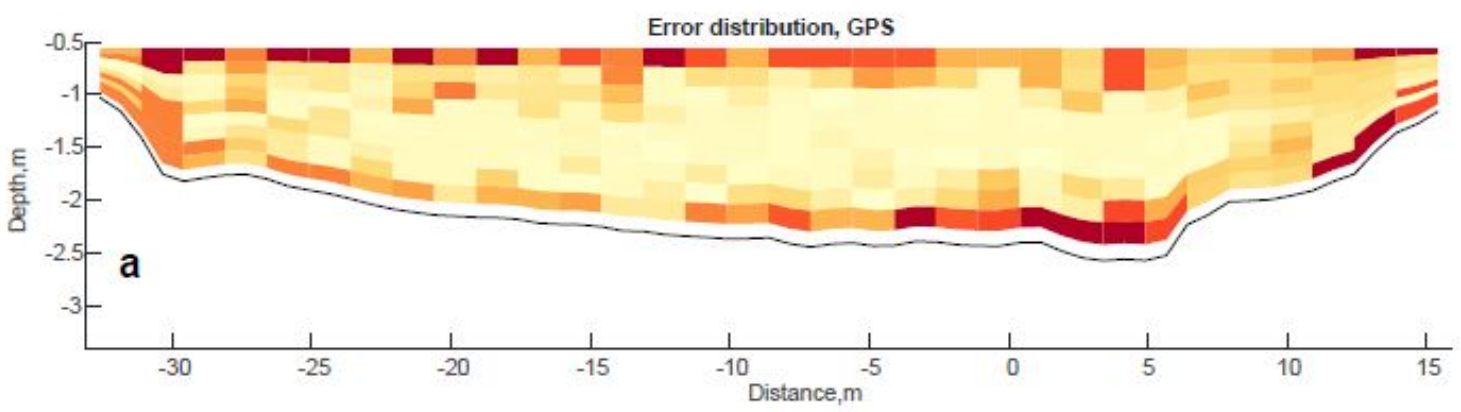

Magnitude of error

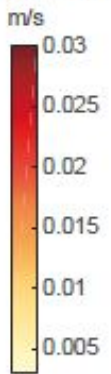

$\mathrm{m} / \mathrm{s}$

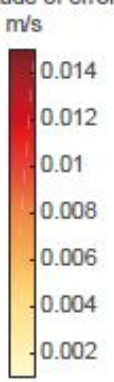

(7)

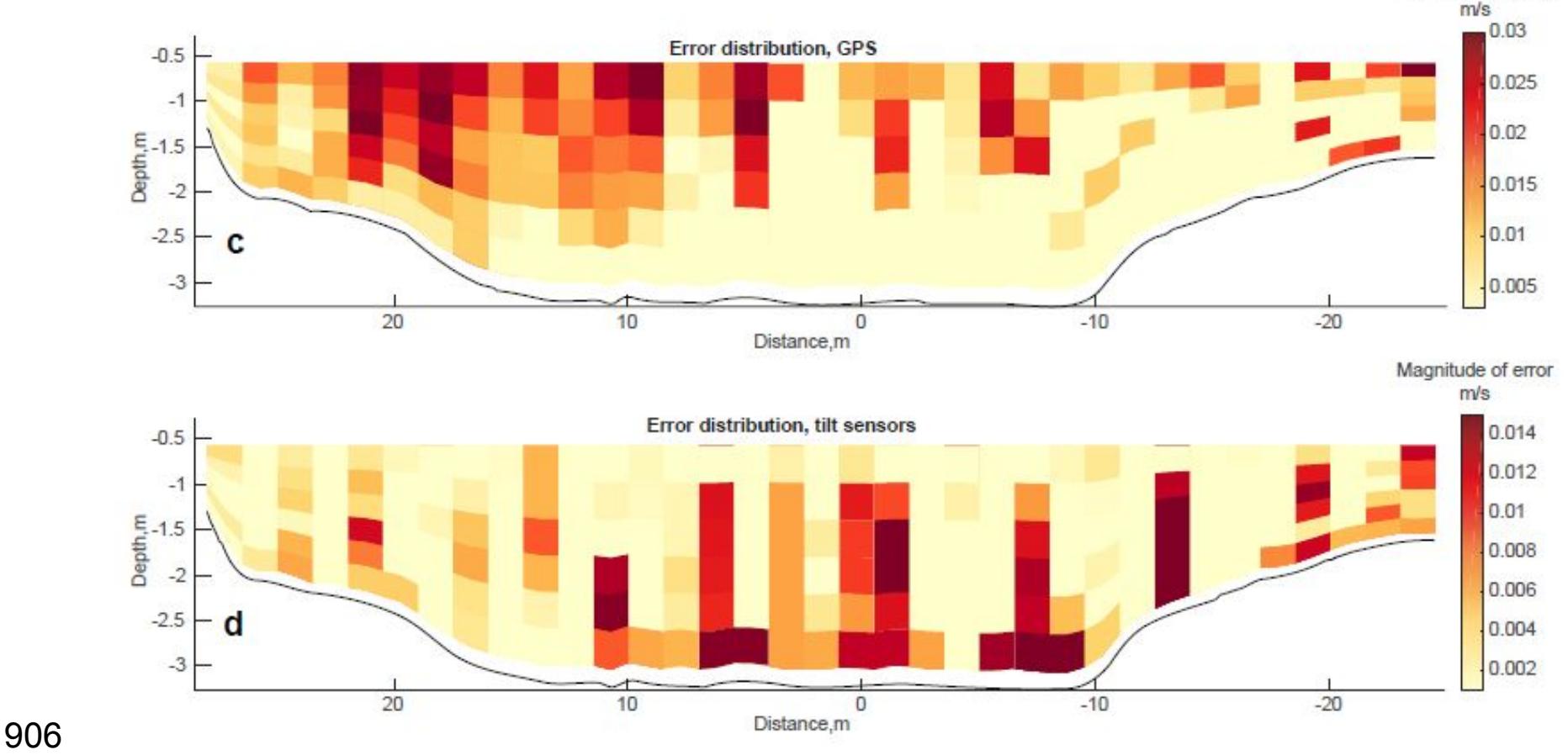

Magnitude of error

907 Figure 12: Error distributions related to GPS for a) Lizerne-Rhône confluence and c) Grande Eau- Rhône confluence, 908 and sensors accuracies for b) Lizerne-Rhône confluence and d) Grande Eau-Rhône confluence, in estimating the 909 secondary velocities using Mmethod $A_{;}$view is looking downstream. 

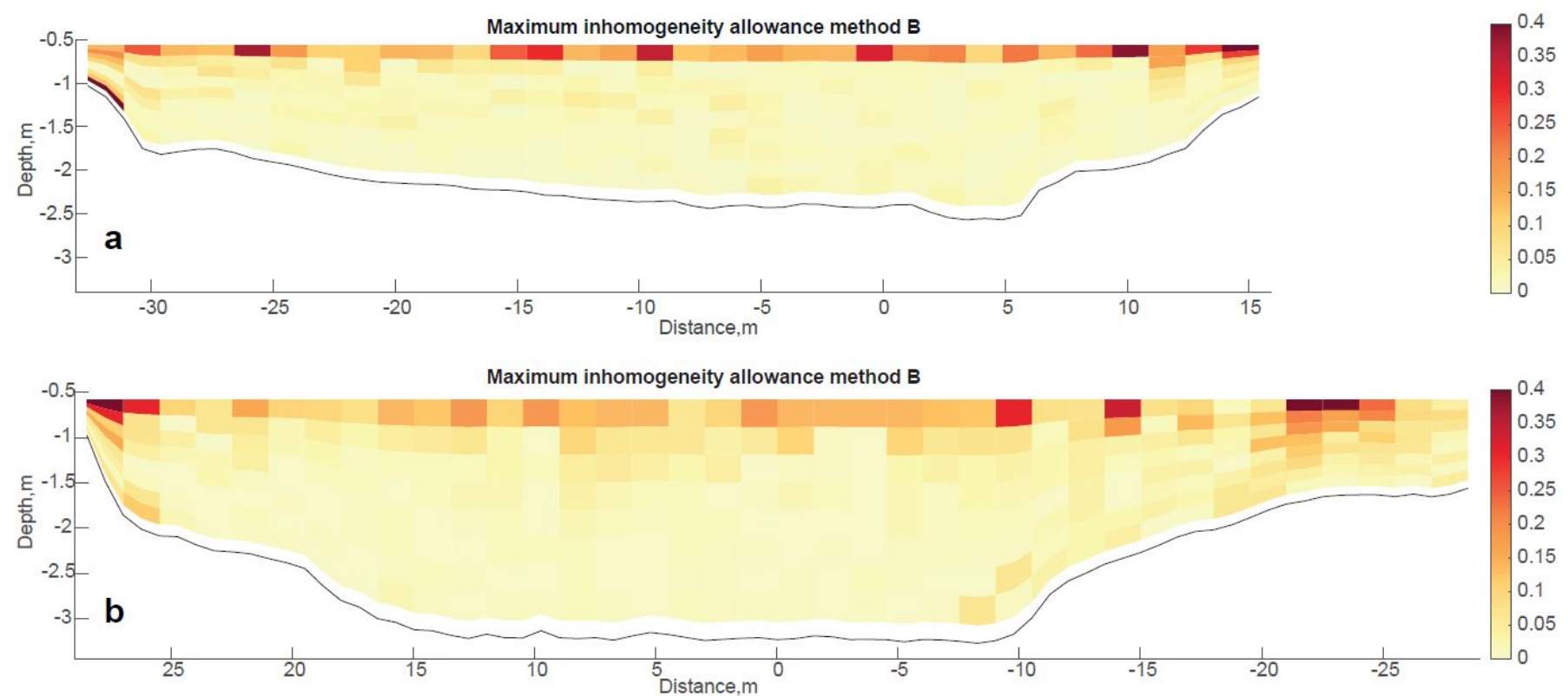

Figure 13: Maximum inhomogeneity allowance ( $m$ ) using Mmethod B for a) Lizerne-Rhône and b) Grande Eau-Rhône confluences; view is looking downstream. 

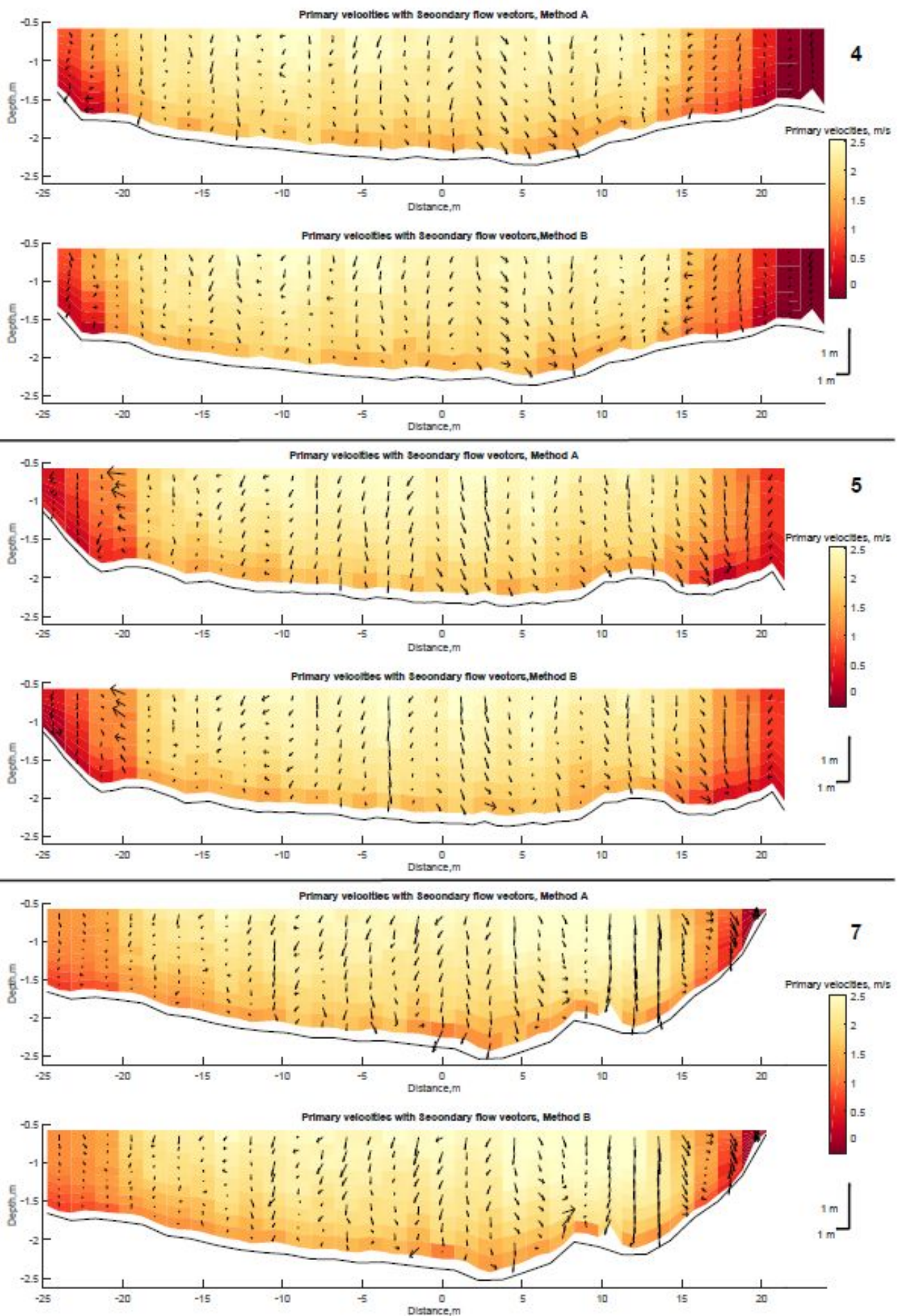

Figure 14: Primary velocities (contours) with secondary velocity vectors estimated using methods $A$ and $B$ at cross sections 4,5 and 7 in Figure 3a at the Lizerne-Rhône confluence; view is looking downstream. 

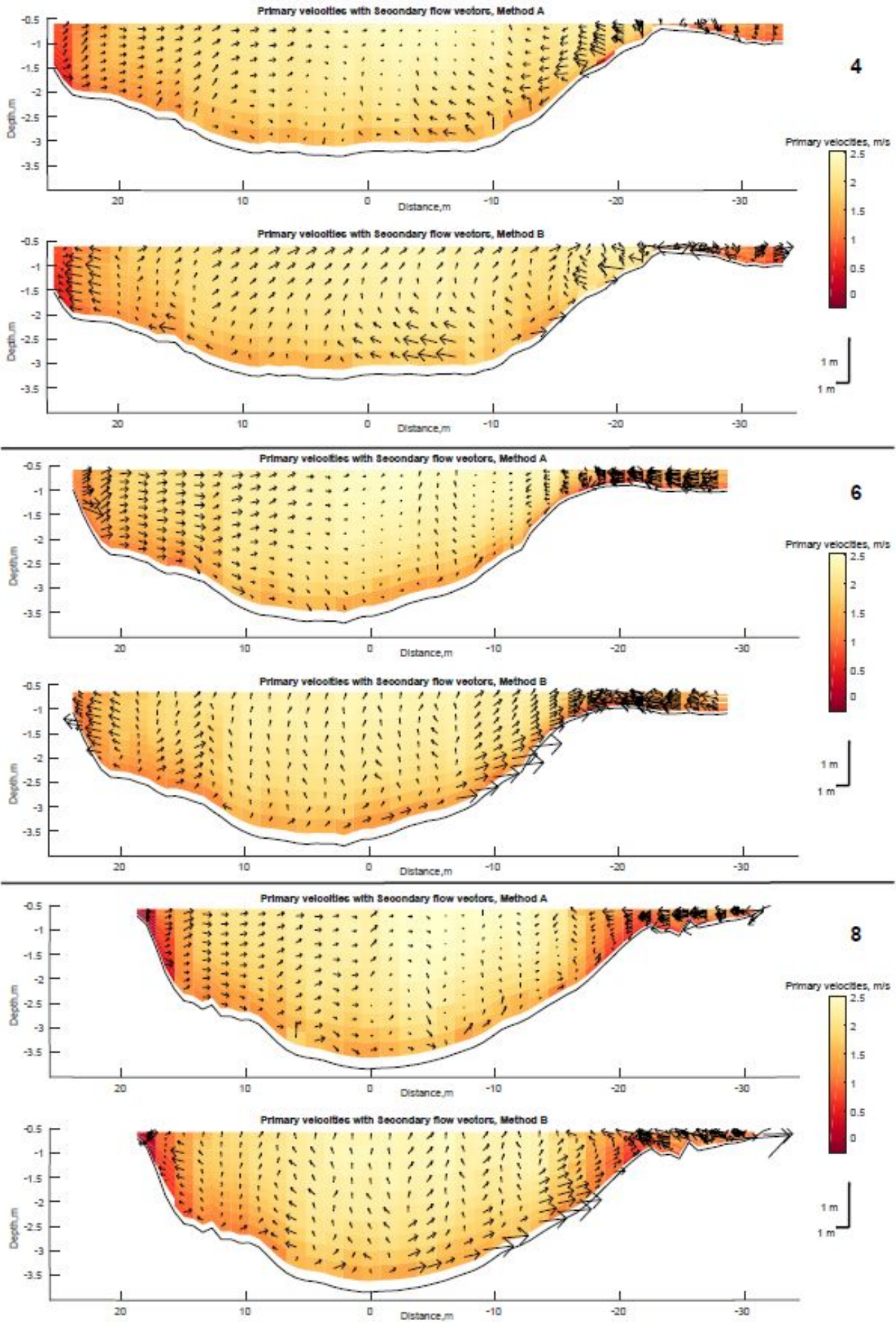

Figure 15: Primary velocities (contours) with secondary velocity vectors estimated using methods $A$ and $B$ at cross sections 4,6 and 8 in Figure $3 b$ at the Grande Eau-Rhône confluence; view is looking downstream. 
a Lateral velocity gradient

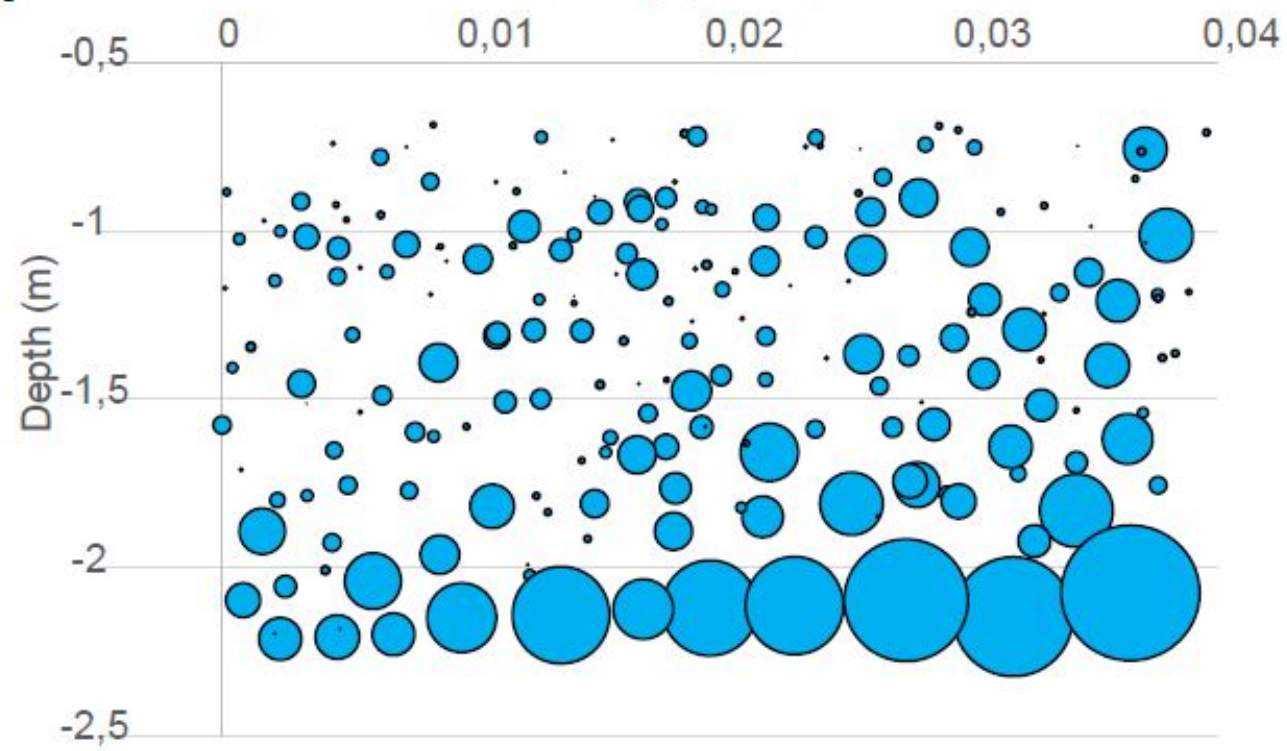

.Relative differences in secondary velocities $(\mathrm{m} / \mathrm{s})$

b

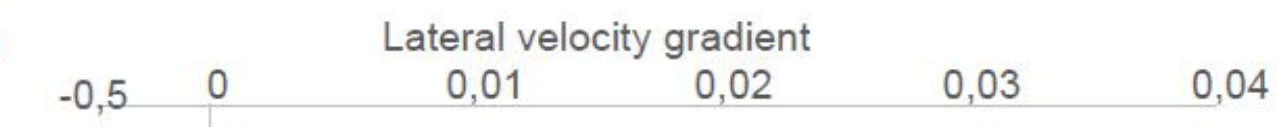

919

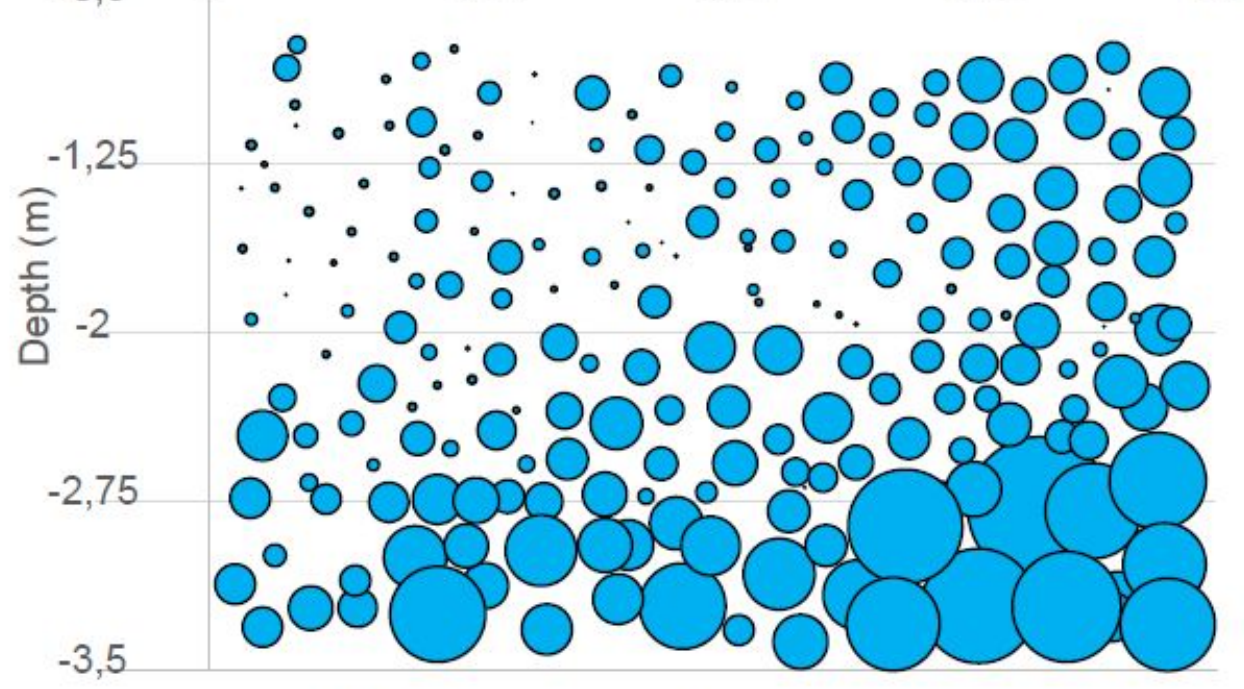

.Relative differences in secondary velocities $(\mathrm{m} / \mathrm{s})$

920 Figure 16: Relationship between lateral velocity gradient, depth and relative differences in secondary velocities for a) the Lizerne-Rhône confluence and b) the Grande Eau-Rhône confluence 


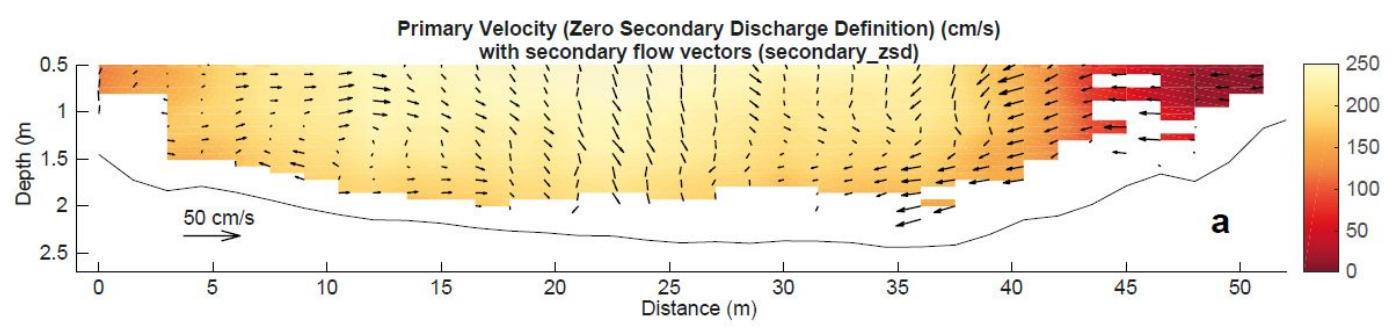

Primary Velocity (Zero Secondary Discharge Definition) (cm/s)

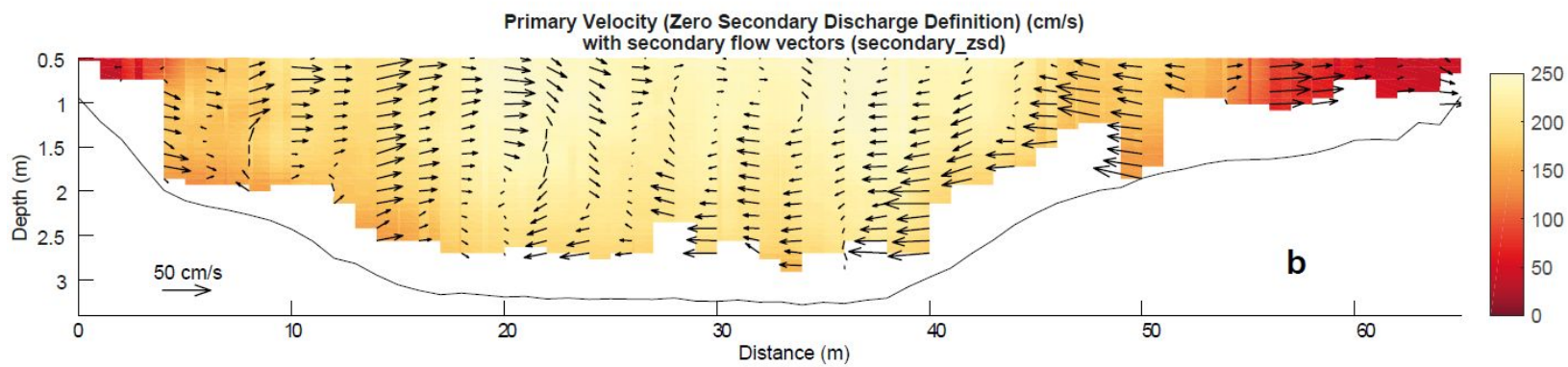

923

Distance $(m)$

924 Figure 17: Primary velocities (contours) with secondary velocity vectors estimated using 925 the VMT at cross section 6 in Figure $3 a$ at the Lizerne-Rhône confluence and at cross 926 section 3 in Figure $3 b$ at the Grande Eau-Rhône confluence. These are comparable with 927 results in Figure 4a/4b for the Lizerne-Rhône and 4c/4d for the Grande Eau-Rhône 928 confluences 


\section{Dear Professor Kirkby,}

Thank you for the decision of moderate revision on our paper ESP-19-0030.R1. We have now been able to undertake the requested changes (marked below in black). We detail our response below (marked below in blue) and we have also supplied a manuscript with changes tracked.

With best wishes

Gelare Moradi for the authors

\section{ASSOCIATE EDITORS COMMENTS}

Thank you for your careful revision which has greatly improved the manuscript. However reviewers have identified a number of points that require some further revision or clarification before acceptance.

Thank you for this positive assessment. We explain our response to these revision requests below.

Reviewer: 1

The revised version of this paper is much improved and the modified analysis presented in the paper focusing on flow within two confluences, rather than downstream of a single confluence, provides a refined basis for comparison of the two different methods ( $A$ and $B$ ) for evaluating flow structure at confluences using ADCP data. The authors are to be commended for undertaking this substantial revision and addressing most of the issues raised in my previous review.

We thank the reviewer for this positive assessment.

The paper now represents an important contribution, but still needs moderate revision to be of publishable quality.

We explain the changes made below.

1) Abstract lines 26-29 The blanket statement here that method $A$ is an improvement over Method B (implied) is somewhat at odds with the conclusion that the two methods have advantages and disadvantages and that both can in the absence of strong shear produce similar results. Also, the extent to which Method A is more accurate than Method $B$ cannot be determined conclusively from a comparison using the two different methods to process the same ADCP data. Some independent measure of the flow using information that is known to accurately represent local flow conditions (such as a dense array of ADV measurements) would be needed to determine whether ADCP data from either method are accurately capturing the flow structure. What seems most appropriate is to indicate that the two methods can, in the presence of strong shear, produce different results, and, given the averaging inherent to method $B$, it is reasonable to assume that Method A should provide more accurate results under these conditions than Method B. In other words, a more tempered statement would seem appropriate given what is accomplished in the study.

This is a very fair point. We have now replaced the end of the abstract with "The comparison confirms that in the presence of strong shear our method produces 
different results to more conventional approaches. In the absence of a third set of fully independent data, we cannot demonstrate conclusively which method is best, but our method involves less averaging and so in the presence of strong shear is likely to be more reliable."

2) Abstract lines 29-32 This statement about counter-rotating cells and scour is not consistent with results presented in the paper. No clear identification of counterrotating cells using method $A$ is presented in the results, nor is a comparison made between counter-rotating cells by methods $A$ versus $B$. These cells also are not related to scour. This statement should be dropped from the abstract and perhaps replaced with a statement that the use of both methods, along with consideration of the factors that influence each method, is valuable for evaluating flow structure at confluences (see point 30 below).

We agree. This has been removed and replaced with "We conclude that it is wise to apply both our method and more conventional methods to identify where data analysis might be impacted upon by strong shear and where inferences of secondary circulation may need to be made more cautiously."

3) Line 118 - irregular cross-sections. I assume this is referring to the irregularity of the bathmetry at cross sections, rather than an irregular alignment of the cross section. This should be made clear.

Agreed - we have inserted "bathymetrically" before "irregular"

4) Lines 126 to 134 If I understand it correctly, given the way the processing calculates the mean cross section, the orientation of any cross section relative to the alignment of the river channel can vary from cross section to cross section along the river. In many fluvial applications the desire is to have cross sections perpendicular to the local channel alignment. For a relatively straight channel, such as the Rhone in this study, that would also imply that cross sections are parallel to one another. The extent to which this condition is achieved seems to depend on the boat tracks and the clouds of bathymetric points produced by these tracks. To what extent did the resulting cross sections for analysis differ from one another and from the alignment of the river?

To some extent this issue is rendered moot by the use of the zero net secondary discharge to analyze the flow structure, but that method also involves rotation of the cross sections. It might be good to show the alignment of the cross sections derived from the processing method and the alignment of the zero net secondary discharge cross sections on figure 3. Cross section alignment can influence the interpretation of secondary flow (see point 7 below).

The reviewer is right to note here that there are two controls on the analysis relating to cross-section orientation: the first is the orientation of data collection which defines the initial mean transect, and this is easier when the main channel is straighter; but this does not necessarily lead to the correct identification of secondary circulation, for which rotation is then needed. To respond to this revision request, we have made two changes.

1. We have added the following at before former line 126 "It is important to note that this mean transect is not necessarily orthogonal to the primary flow direction and so will not yield true primary and secondary flow estimates without further correction. We address this below."; and just after reminded the reader that the initial bathymetric model is the initial mean transect. This 
5) Lines 326-329 The decision to use six cross sections seems to be based on the analysis of standard deviation of the velocity data. Although this is presented in the results, this basis should at least be mentioned here.

Agreed - we have added "Hence, in this paper, data are processed for cross-section 6 at the Lizerne-Rhône confluence (Figure 3a), and for cross section 3 at the Grande Eau-Rhône confluence (Figure 3b). Identification of the minimum number of repeat transects necessary per cross-section was undertaken using cross-section 9 at the Lizerne-Rhône confluence (Figure 3a), which involves 16 repetitions. We noted that after application of Method A, the standard deviation of velocity stabilized with six repetitions, which is the number we adopt for this study."

6) Lines 363-368 why was a standard deviation of plus or minus 1 degree chosen as a reasonable value for tilt sensor error? Also what was the standard deviation of the displacement error distribution? How was this determined?

We have added "based on manufacturer specifications" for the tilt error; and "measured by the dGPS for the dGPS positions (as a function of satellite configuration during measurement)" for the positions.

7) Lines 380-381 The zero net secondary discharge method was used to determine secondary flow. This choice can have an influence on the depiction of secondary flow compared to other secondary-flow depiction methods (e.g. cross-stream perpendicular to the local channel alignment, Rozovskii method, maintenance of flow continuity of between cross sections). This issue should at least be mentioned in the discussion or conclusion (i.e. that this aspect of data processing, not just the ADCP operation, is a relevant one for producing differences in secondary flow patterns, but is not considered in this study).

This is correct. We use the zero net secondary discharge method as work two decades (Lane et al., 2000) showed that other methods (e.g. Rozovskii) are not correct (Rozovskii, for example, means that the primary flow direction changes within an individual cross-section). To make this clear, we have made two changes

1. In the methods section we now write "In order to distinguish between primary and secondary components of flow, we need to rotate the initial mean transect. Options for doing this are reviewed in Lane et al. (2000) and we do not assess them here, but rather apply the zero net cross stream discharge definition (Lane et al., 2000)."

2. We have added at the end of the Discussion "Finally, we wish to emphasise that the impact of averaging is only one element that must be considered in obtaining reliable primary and secondary clow estimates in river confluences. Other issues, such as the rotation method needed to distinguish primary and secondary circulation, remain important and should be considered routinely."

8) Lines 391 to $400 \mathrm{It}$ is not clear why angular rotations are needed for vertical velocities. A rotation should not be necessary if the cross section is a plane aligned from the flow surface to the bed. 
No - we disagree here. Secondary velocity is defined as that component of velocity that is orthogonal to the primary flow direction and this includes components that will be both predominantly lateral and vertical. The next flux associated with both lateral and vertical fluxes should be zero for true definition of the primary velocity, which is why this rotation is needed. We have made this clear by adding "secondary circulation is all flow that is orthogonal to the primary flow and not just horizontal flow; there should be not net secondary flux in a section; and so correction should also consider vertical velocities".

9) Line 401 What does curvature of cross-sections refer to here?? How are the cross-sections curved?

We have clarified this by adding "correct for weak curvature with the survey method at the edges of each transect line (e.g. Figure 3),".

10) Lines 410-411 this statement about differences is not put into any context and seems to contradict the previous sentence that the velocities are similar for the two methods

Agreed - modified to "and the differences in estimated secondary flows are minor. The differences are most pronounced between -10 and $5 \mathrm{~m}$, in the middle of the main channel'.

11) Figure 4 - the caption for this figure appears to be incorrect. The top two frames $(a$ and $b$ ) are for one confluence and the bottom two ( $c$ and $d$ ) are for the other. The caption is confusing as written.

Corrected to "Figure 4: Primary and secondary velocities estimated for the LizerneRhône Method A (a) and Method B (b) and the Grande Eau-Rhône Method A (c) and Method B (d)"

12) Table 1 It would be good to include the velocity ratio (ratio of mean velocities) in this table as this should provide the most direct information on the difference in the magnitude of lateral fluid shear between the two flows. Based on the arguments in the text this ratio should be much larger for Grand Eau than for Lizerne.

This may be correct if the two tributaries had beds at the same altitudes, but there is also a very marked difference in tributary elevations and hence depth ratios. This means that the velocity ratio is not a useful parameter to report. This was not clear in our previous version of the paper and so we have now made this clear through the following changes:

1. Where we introduce the Lizerne, we have modified the description of the junction angle to: "It reaches the Rhône between Ardon and Vétroz, forming a $90^{\circ}$ junction angle and it has a bed that is nearly concordant with the Rhône." - and where we introduce the Grande Eau, we now write: "The Grande Eau bed is c. $1.5 \mathrm{~m}$ higher than the Rhône such that it is markedly discordant".

2. In the results, we now mention the importance of bed discordance with the relevant sentence modified to: "This is due to a high degree of bed discordance between the Grande Eau and the Rhône, which increases the penetration of the tributary flow into the main channel over the junction, and which forms a zone of high lateral and vertical shear, on the one hand, and main channel narrowing because of penetration of the tributary point bar on the other hand." 
3. When we now discuss Figure 7 we have added in reference to vertical and lateral shear.

13) Line 415 reference figures $4 \mathrm{c}$ and $\mathrm{d}$ here Added

14) Line 421 and 422 above the junction? Flow enters at the junction. This phrase can be deleted.

Clarified - it was meant to be vertical. We now use "over" rather than "above".

15) Line 423 tributary point bar should be change to tributary mouth bar. Point bars occur in meandering rivers

Changed

16) Line 427 inner bank? Not clear which bank this is. Assume the left bank but it should be specificied as there is no clear inner and outer bank

Changed to "bank on the tributary side of the channel".

17) Line 456 to to 459 It is not entirely clear what cell is being referred to here as the "described above" is disconnected by many lines of intervening text. Recommend it be explicitly reidentified here.

We have rewritten the sentence as "Indeed, the marked differences between methods $A$ and $B$ at the Grande Eau confluence (Figure 7) are also in a zone of strong lateral shear." to make reference back to Figure 7.

Also the use of the term cell for secondary flow and cell for the mesh can be confusing at places. May want to consider using mesh cell and secondary cell rather than just the term cell.

This is an excellent point - there are actually 3 cells: the aDcp, the mesh (numerical, that we use for the analysis) and secondary circulation. For all mentions of cell we now distinguish between these.

18) Line $475-478$ the stabilization of the variance is likely a product of the number of measurements contained within the each mesh cell. Do methods $A$ and B produce different numbers of measurement points within each mesh cell? It seems likely they would given that method $A$ should produce many more individual velocity readings than method $B$. This might be an important factor in stabilizing variance.

This is right and we have clarified it - adding, "and this is likely because method B uses fewer measurements per mesh cell."

19) Line 527 mouth bar Modified

20) Line 521-523 careful with language here. Method A cannot produce stronger penetration or weaker upwelling of the flow. It indicates that secondary velocity components differ from those depicted by Method B, which has implications for the strength of penetration and upwelling.

Yes - and so we have modified the text in 6 places to make sure we mean identification rather than production. 
21) Line 546 would be good to present velocity ratio in table 1 to confirm this. Also information from the primary velocity data on the maximum lateral shear gradients for the shear layers in each confluence would also be useful.

This is a useful point and did need some clarification. As noted above, the issue is more that the GE-R has a more complex shear zone (with lateral and vertical shear) rather than necessarily a more intense lateral shear zone. To capture this point, we have rewritten the section as "the Grande Eau-Rhône has a more complex shear zone, likely due to the effects of bed discordance, and there are more significant differences in the estimation of primary and secondary velocities"

22) Line 557 As mentioned with the abstract, this statement should be qualified. One can reasonably assume it should provide more accurate information on the secondary velocities, but this cannot be conclusively confirmed without independent corroborating evidence.

Yes - this is a very fair point and to follow our changes to the abstract, ee have modified the sentence to: "In this case, as method A involves less spatial-averaging than method $B$, it may provide more accurate information on the flow behavior, but such a conclusion really needs a third and independent method to confirm this conclusion."

23) Line 564 low levels of lateral shear acceptable for using method $B$ ?? Given our response to point 21, we have modified this sentence to: "At the LizerneRhône confluence, even though the momentum ratio is similar to Grande Eau-Rhône confluence, there is only more localized shear in the flow and a simplified shear zone (Figure 9). In such a situation, using method B to detect the large scale patterns of secondary flow may be more advantageous, because it involves more spatial averaging."

24) Line 569 velocity ratio and max values of lateral velocity would help confirm the absence of lateral shear.

See above - lateral shear is not, in our view, the only issue (it is already shown also in Figure 9 - it is more the complexity of the shear zone arising from both lateral and vertical shear. We hesitate in adding more quantitative data because it might lead to others applying an overly simplified rule when the magnitude of shear likely to lead to method $A$ being needed also varies with the distance of the shear zone from the sensor. We now make this point explicitly by adding "It may be tempting to introduce some kind of shear or velocity gradient threshold to identify when Method A might be preferable. To do so could be misleading as this value will also depend on the distance of the shear from the aDcp."

25) Line 568-573 run-on sentence need to revise this by at least splitting it into two sentences.

Sentence split into two

26) Line 579-584 Again a rather long sentence. Also it is best to use an even number of transects to avoid potential directional bias in GPS signals

The sentence is now split and we have added "We also note that an even number of repeats may be important to avoid directional bias in dGPS positions." 
27) Line 599-611 This section on VMT is underdeveloped and gives the impression of material that has been inserted into the paper as an afterthought. The comparison is only for a single cross section and generalizations should not be drawn on the basis of this comparison, even suggestively, especially given that VMT has provided high-quality depictions of secondary flow at confluences in many instances. It is also not clear why the results of the VMT analysis include larger areas near the bed without data. Moreover, the statement about VMT using a straight mean crosssection is confusing. Don't methods $A$ and $B$ also use straight mean cross-sections? It seems best that this rather superficial comparison with VMT be deleted from the paper. Doing so will not detract from its main message of the paper. Generally VMT results should be consistent with Method $B$, although the level of spatial detail may be greater in VMT depending on the size of the cells in relation to bin size.

We agree and so have removed completely this text, including Figure 17.

28) Lines 615-618 can some indication be provided here about what constitutes a small river versus a large river (would avoid the use of the term "big" since that term is often associated with mega rivers such as the Amazon and Congo)? Use of small versus large is rather subjective and it would be helpful to have at least some metrics associated with these terms.

This is a good point and needed three changes to be made. First, we are now explicit that what we think matters here is depth (and hence shallower versus deeper).

Second, we don't have enough cases to specify what this depth is and so we simply state relative to our studied confluences in the modified text.

These two changes are now included in this statement: "This is likely to be the case particularly in rivers shallower than those studied here and where high resolution is required due to large velocity gradients. In rivers of the scale studied here, and deeper, by increasing the mesh cell size, we can still have sufficient data to estimate velocity vectors, and the effects dGPS and tilt sensor errors have a minor effect." The third change relates to the point that we don't have enough data either to identify specifically what we mean by big and small but also to urge caution, following the difficulty if identifying critical values of shear. What is big (so Method A) and small (so method B) will also depend on shear. To capture this point we have added: "We are not yet in a position to identify the depth at which Method A becomes preferable to Method B, and again this will depend on other parameters such as the intensity of shear and so may not be readily generalizable between confluences."

29) Line 631 again velocity data would be useful for evaluating shear See arguments made above.

30) It might be appropriate to recommend that it can be useful to use both methods to analyze flow structure at confluences, as this paper has done, to see how they differ. If they do not differ greatly this provides reinforcement that the depicted patterns are probably accurate. If they differ, consideration should be given to the factors that can produce differences between the two methods, and a preference for one depiction over another weighted according to the prevalence of these factors. This is an excellent suggestion and conforms with our Conclusion. Our response to point 24 partly makes this conclusion.

We have added the following to capture this argument: 


\begin{abstract}
"We are not yet in a position to identify the depth at which Method A becomes preferable to Method B, and again this will depend on other parameters such as the intensity of shear and so may not be readily generalizable between confluences.

The difficulty of identifying the depths of rivers and intensities of shear that make one method preferable over another precludes adoption of simple quantitative guidance on which method to use when. As both methods have some disadvantages, we argue that both methods should be applied. If they give similar results, then there should be confidence in both. If and where they differ, analysis should be undertaken to identify why, and hence which method is likely to be preferable. Association of the differences in primary and secondary velocities inferred between the two methods with estimates of shear intensity and with estimated tilt and positioning errors should then help decide whether Method A or Method B is preferable in a particular case. This preference may vary between confluences but also through time at a confluence, if shear or flow depth changes significantly between survey dates."
\end{abstract}

Reviewer: 2

Comments to the Author General comments

The reviewer appreciates all the answers and effort made by the authors to address all suggestions and comments made by reviewers in the new manuscript submission, which has significantly improved the manuscript.

We thank the reviewer for this positive assessment.

Before publication, I suggest the authors clarify some specific questions detailed below.

Note: As a suggestion, in order to the proposed methodology is easily apply by ADCP users, I strongly recommend the development of an open source code. To include these methods into the widely used VMT software will be an excellent tool for ADCP users interested on flow structures estimation at complex hydrodynamics zone such as confluence, bifurcation, bends, etc...

We think the best way to achieve this is to integrate the method into the VMT and we are currently in discussion with the VMT developers to do this. We can also make our own code available upon request and have added this to the acknowledgements.

Specific Comments:

Table 1: discharge from Rhone and tributary rivers are not provided. Added

Figure 4: caption references do not agree with figures and text. Corrected in response to Reviewer 1.

Figure 4: I suppose sections are looking downstream. Clarify in caption. Clarified, and in all other captions

Line 411-412: there are other verticals with similar differences. Could the authors justified in more details? 


\section{Typo: - $5 \mathrm{~m}$ should be $5 \mathrm{~m}$ - corrected; this then deals with this concern}

Line 416-417: It was confuse to me that this affirmation is valid for primary velocities but secondary component look opposite (i.e. at shorter distance from ADCP). Could you clarify?

We don't make this affirmation for secondary velocities. This difference, though, is partly explained because it depends on the magnitude of primary velocity relative to secondary velocity. We don't think this needs to be explained as we don't discuss secondary velocities in this way.

Line 421-428: the sentence is very confused. Could the authors rewrite this sentence?

Clarified in response to the request from reviewer 1 (point 14)

Moreover, Grande Eau-Rhône streams have difference densities that could explain the penetration of Grande Eau river into Rhone near bed? It will be useful for reader to have this information to understand the non-common secondary pattern processes presented at this confluence.

We don't believe this is an issue as the differences in suspended sediment load and temperature were negligible during measurement. We prefer not to get into this issue as it would detract from the paper.

Figure 4 and 7: I cannot see an agreement between Figure 4c,d and Figure 7b,d. For example, a clear differences in secondary velocities intensity is presented near water surface at distance between 0 to -10 and lower discrepancy between distance 20 to 10. However, Figure 7 shows the opposite behavior.

This is simply a color scale effect - Figure 7 plots magnitudes of difference whereas Figure $4 \mathrm{c} / \mathrm{d}$ show absolute values. The zone described above suggests differences of around $0.1 \mathrm{~m} / \mathrm{s}$ in Figure 7 which scales with the differences shown in the same zone by comparing vector lengths Figures $4 \mathrm{c}$ and $4 \mathrm{~d}$. No changes made.

Figure 6: add legend in figure 6a.

We have deleted the figure $6 \mathrm{~b}$ legend

Line 495-497: This sentence is not clear to me. Why the relation between ship movements and uncertainties in dGPS data produce a large error near surface and not in all water columns?

This was unclear - and so we have rewritten it as "Near the surface, as there fewer measurements that can contribute to the estimation of aDcp position and tilt, uncertainties in dGPS data will have a greater effect."

Line 608-612: it is surprising to me the big difference between Method $A$ and $B$ with VMT (similar that is doing by methods $B$ ). How many transects were used to obtain figure 17b using VMT? In order to compare the methods should be the same amount than Figure 4. Clarify.

The VMT comparison has been removed. 
Table 1: Selected upper Rhône tributaries with their typical characteristics

\begin{tabular}{|l|c|c|}
\hline Sites & Lizerne & Grande eau \\
\hline Tributary upslope contributing area $\left(\mathrm{km}^{2}\right)$ & 64.8 & 132 \\
\hline Main stem upslope contributing area $\left(\mathrm{km}^{2}\right)$ & 3401 & 5088 \\
\hline Basin area ratio & $1.89 \%$ & $2.59 \%$ \\
\hline Tributary width $(\mathrm{m})$ & 6.5 & 16.5 \\
\hline Main stem width upstream of junction $(\mathrm{m})$ & 46 & 58 \\
\hline Width ratio & 0.15 & 0.28 \\
\hline Junction angle $\left({ }^{\circ}\right)$ & 80 & 70 \\
\hline Tributary Froude number & 0.32 & 0.05 \\
\hline Bed slope of the tributaries upstream of the confluence $(\%)$ & $\sim 0.5$ & $0.5-1$ \\
\hline Main stem slope upstream of the confluence $(\%)$ & 2 & 2.2 \\
\hline Tributary slope $\left({ }^{\circ}\right)$ & 33.1 & 26.6 \\
\hline Rhône discharge during measurement $\left(\mathrm{m}^{3} \mathrm{~s}^{-1}\right)$ & 182 & 300 \\
\hline Tributary discharge during measurement $\left(\mathrm{m}^{3} \mathrm{~s}^{-1}\right)$ & 4 & 8.13 \\
\hline Discharge ratio during measurement & 0.022 & 0.027 \\
\hline Momentum ratio $($ Mr) during measurement & 0.018 & 0.022 \\
\hline
\end{tabular}


Figure 1: Bed elevations, the best fit to those elevations and the water level representation

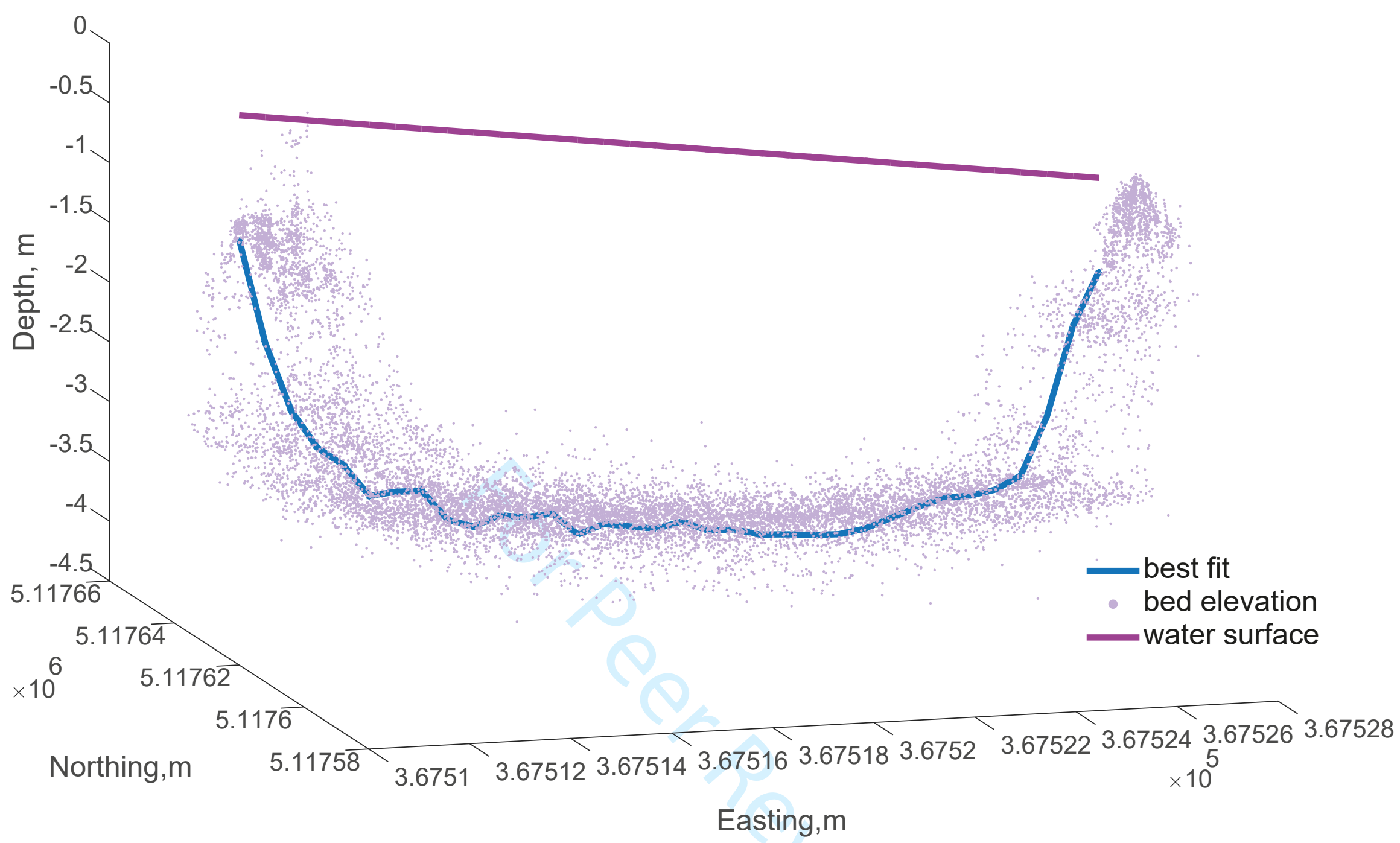


Figure 2: An example of beam velocity positioning within each mesh cell, using method $A$, Dots show beam velocities and color is an automatic Matlab function to distinguish between different cells

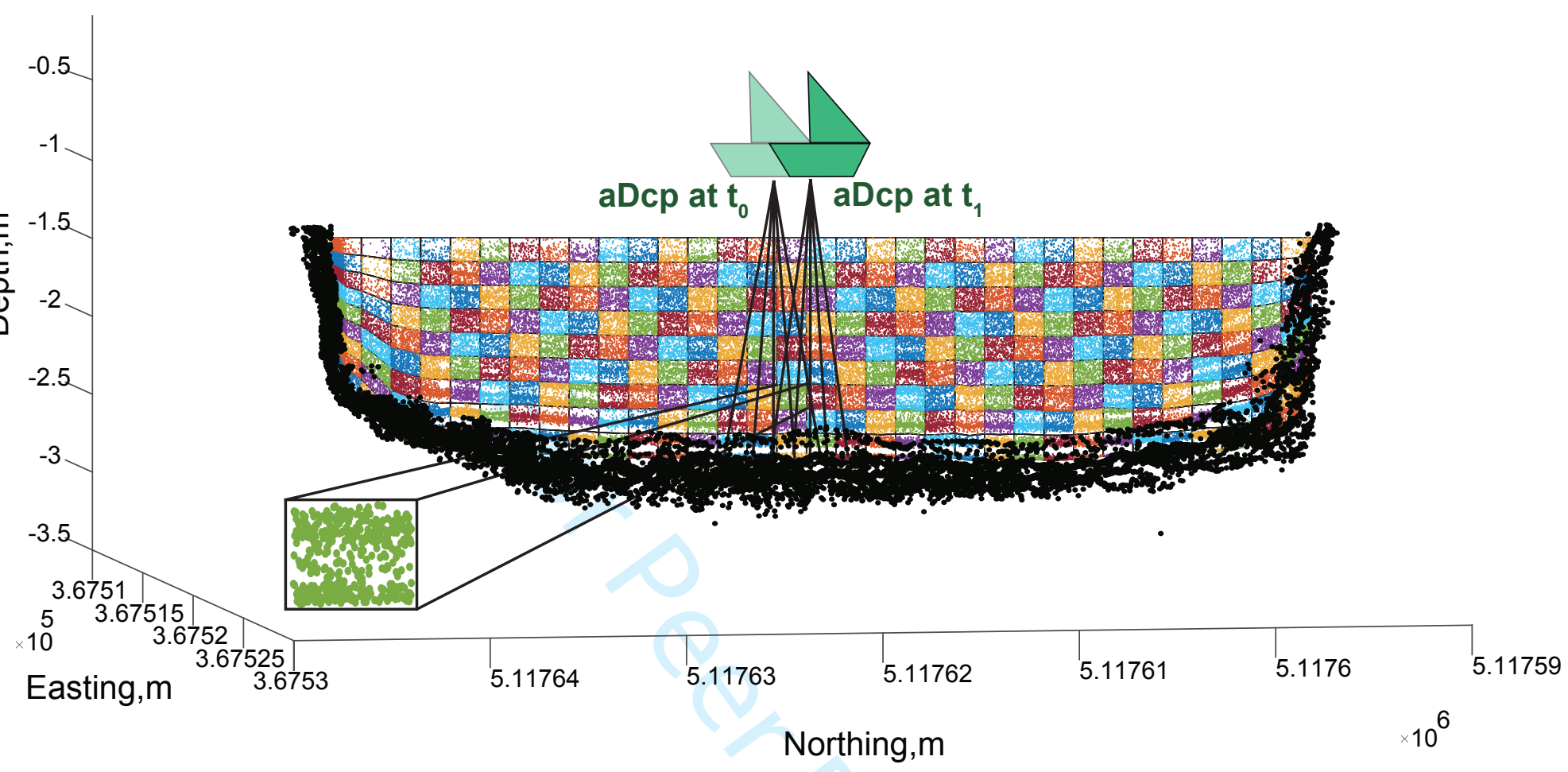


Figure 3: Tracks navigated by SonTek aDcp moving boat system at a) Lizerne-Rhône confluence near Vétroz, at 07/07/2017 and b) Grande Eau-Rhône confluence near Aigle at 23/05/2018. The repeated transect data assessed in this paper are from cross-section 6 at the Lizerne-Rhône confluence and cross-section 3 at the Grande Eau-Rhône confluence and c) Rope-Pulley system

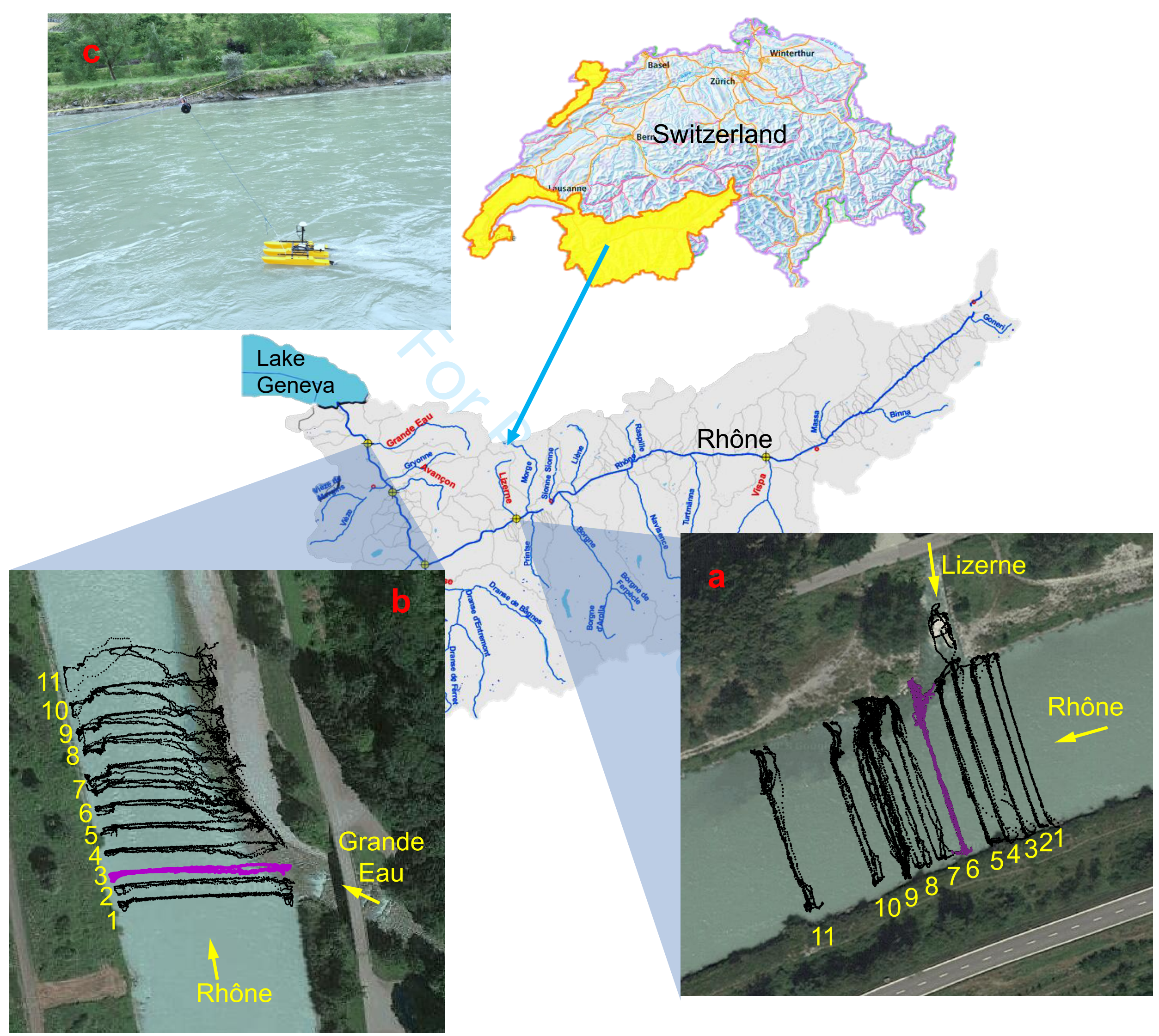


Figure 4: Primary and secondary velocities estimated for the Lizerne-Rhône Method A (a) and Method B (b) and the Grande Eau-Rhône Method A (c) and Method B (d); view is looking downstream.
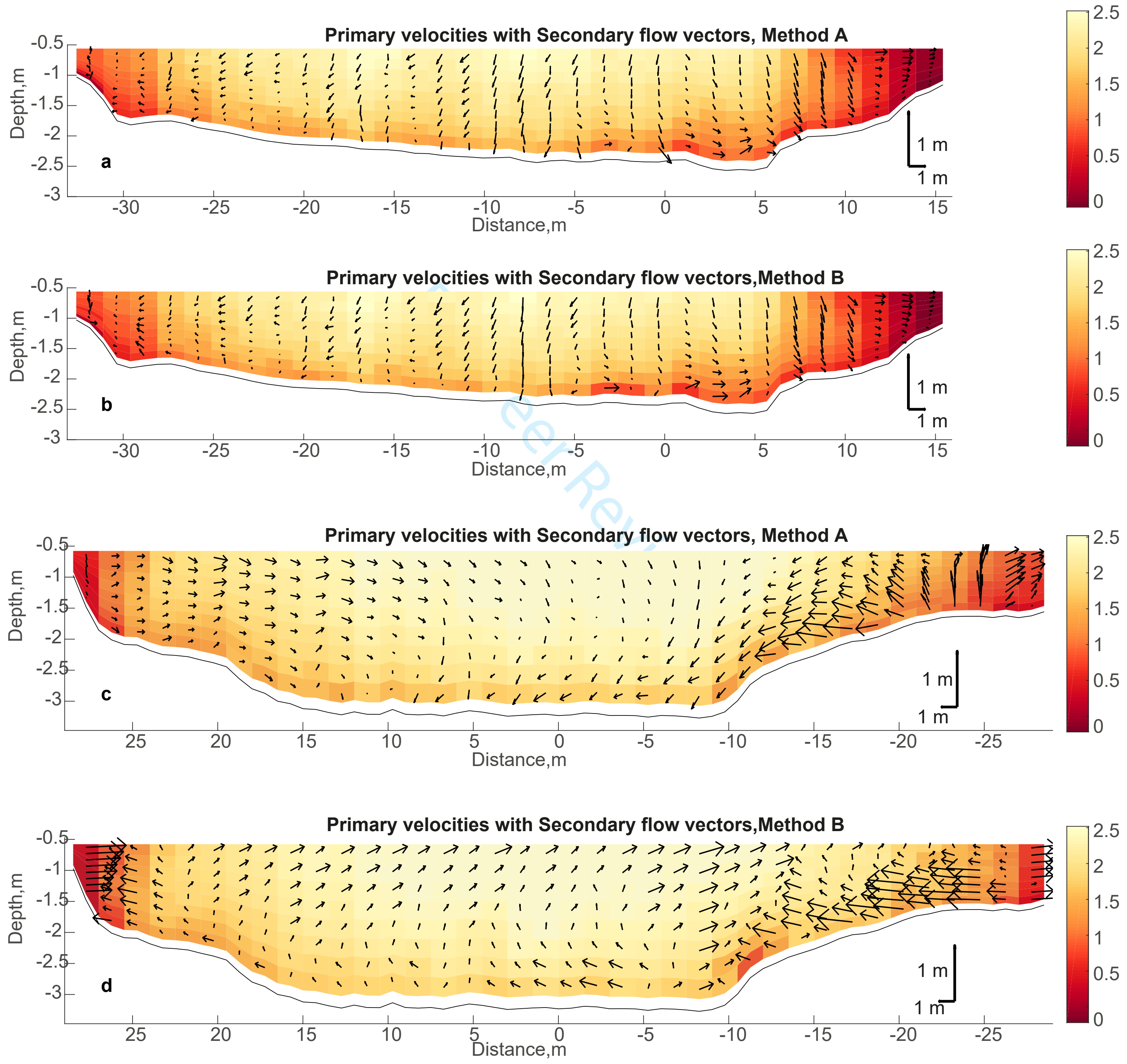
Figure 5: Differences between magnitude of primary and secondary velocities $(\mathrm{m} / \mathrm{s})$ between methods $A$ and $B(a$ and $b)$ and the percentages of their difference $(\mathrm{m} / \mathrm{s})$ (c and d), at the Lizerne-Rhône confluence, for cross-section 9; view is looking downstream.
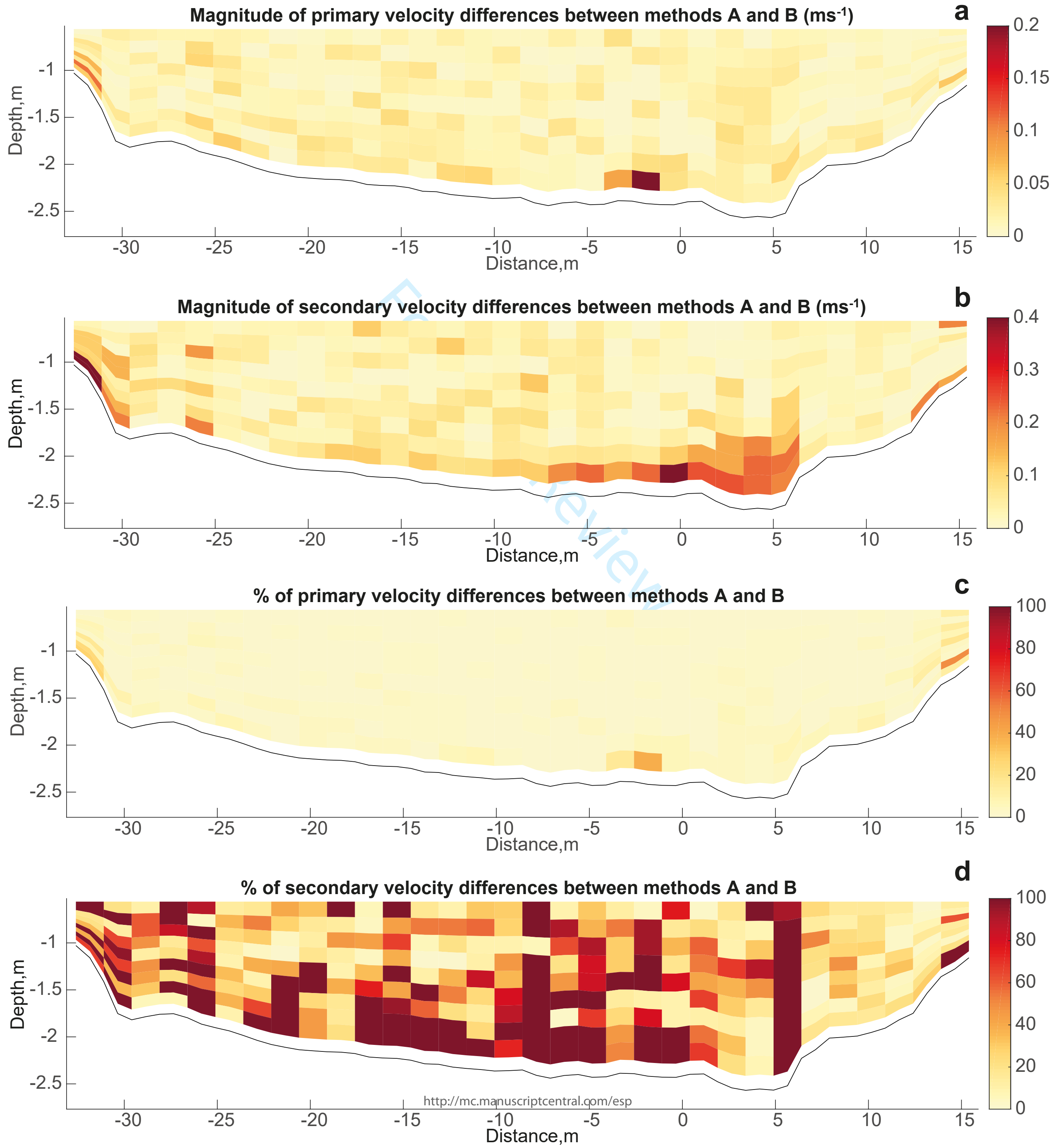
Figure 6: Relative differences in a) primary velocity magnitudes and b) secondary velocity magnitudes, between methods A and B, at the Lizerne-Rhône confluence, for cross-section 6
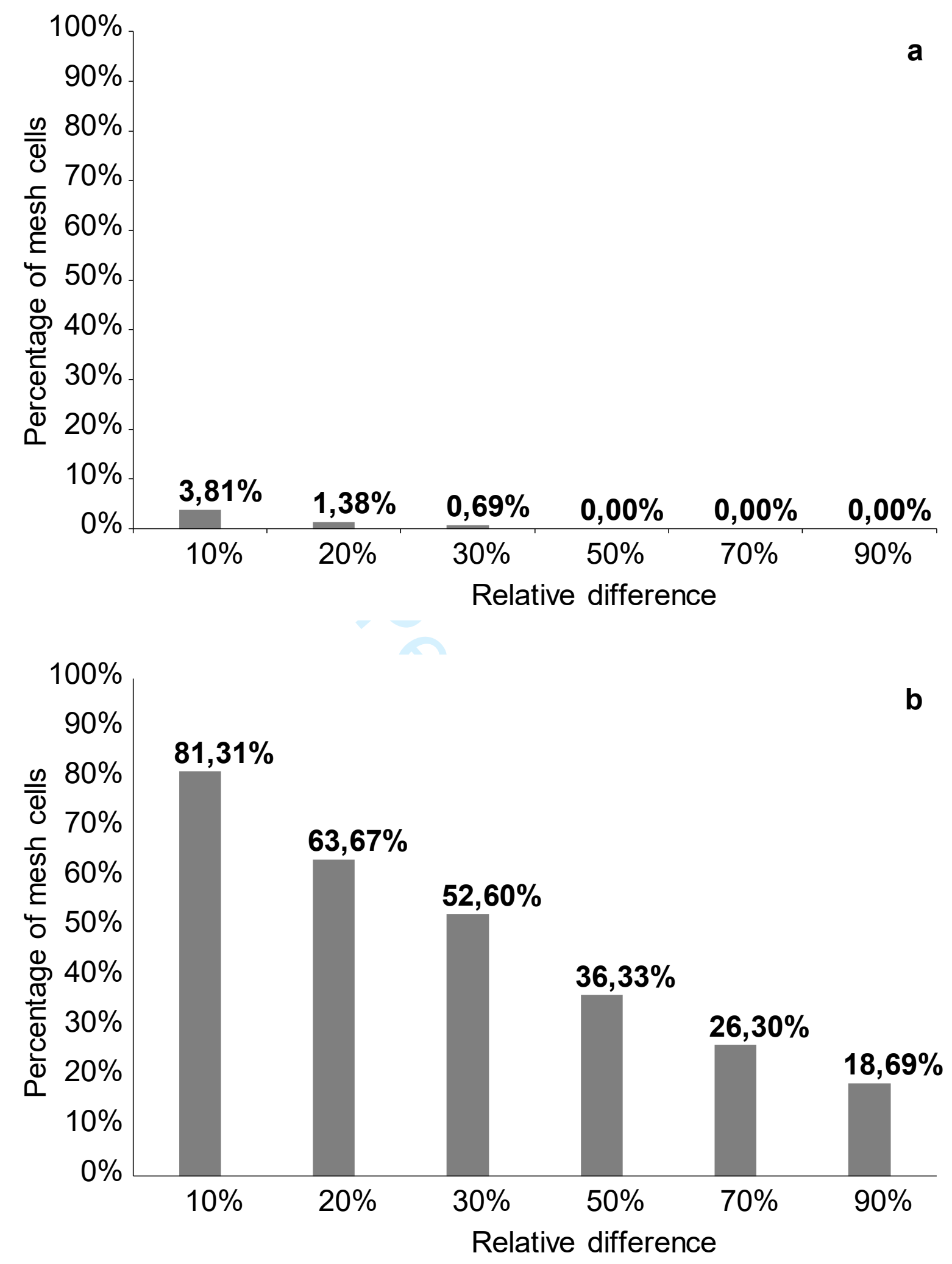
Figure 7: Differences between magnitude of primary and secondary velocities $\left(\mathrm{ms}^{-1}\right)$ between methods $A$ and $B(a$ and $b)$ and the percentages of their difference (c and d), at the Grande Eau-Rhône confluence, for cross-section 3. view is looking downstream.
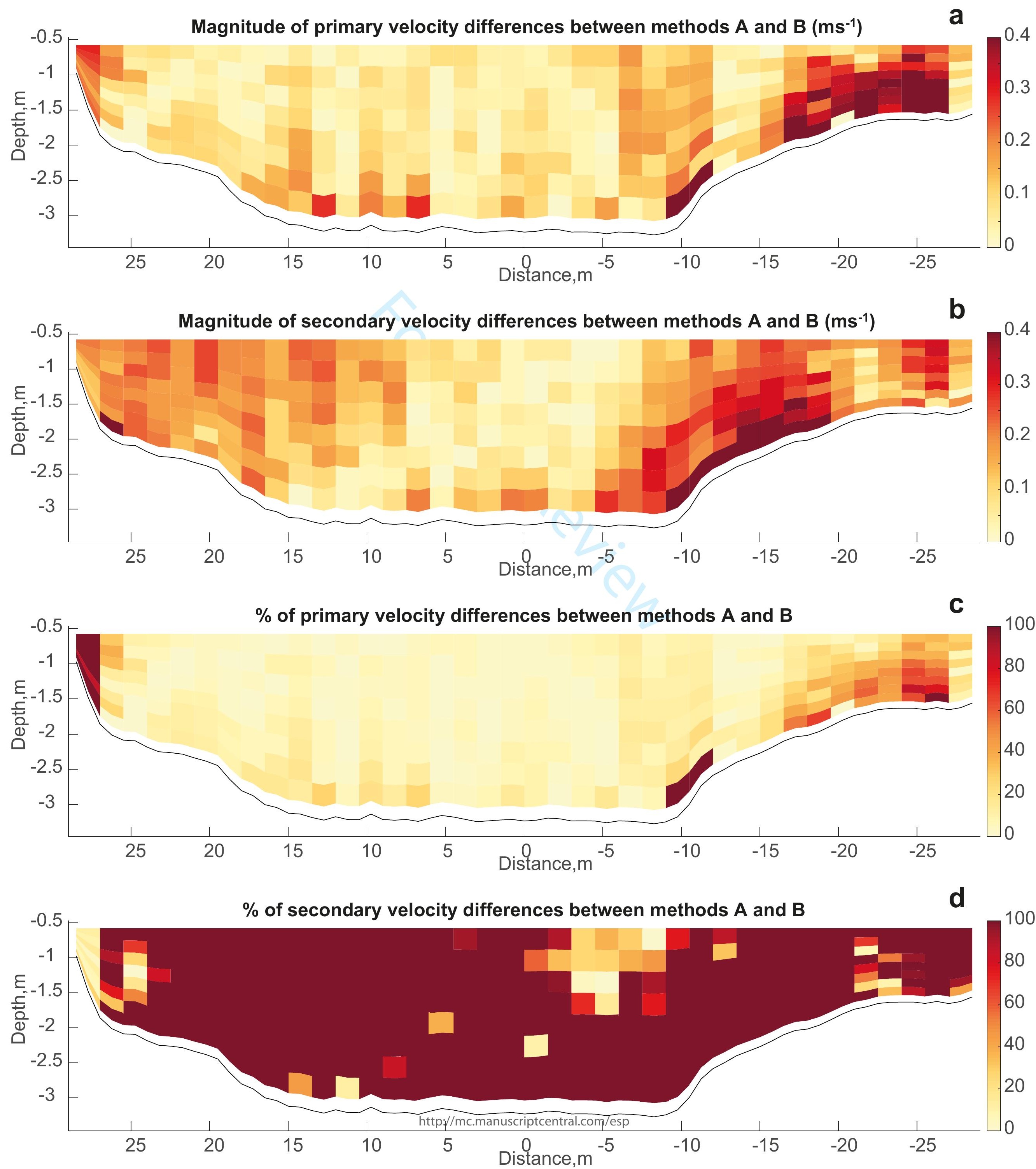
Figure 8: Relative differences in a) primary velocity magnitudes and b) secondary velocity magnitudes, between methods A and B, at the Grande Eau-Rhône confluence, for cross-section 3, view is looking downstream.
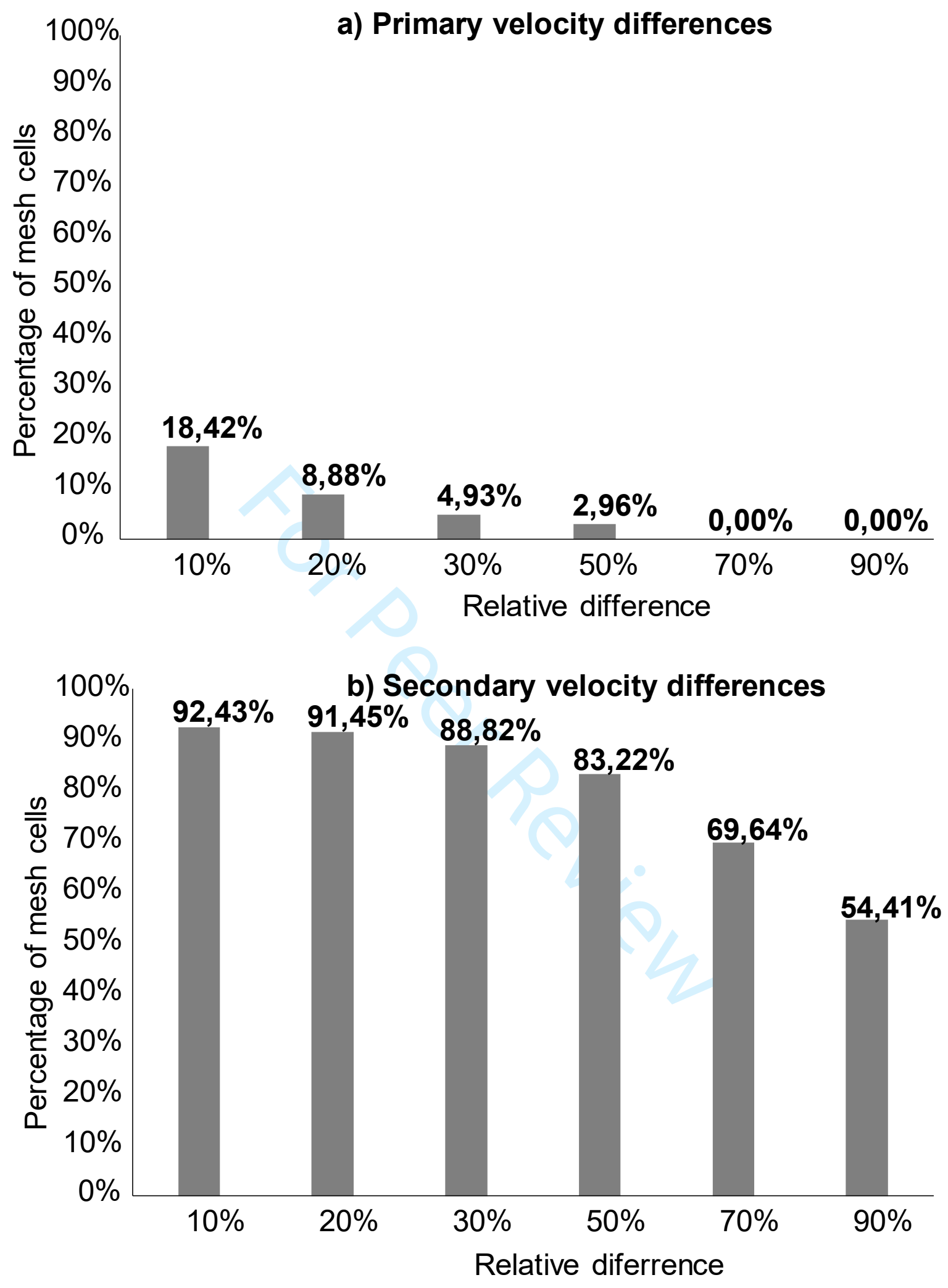
Figure 9: Lateral velocity gradients $\left(\mathrm{s}^{-1}\right)(\mathrm{a}, \mathrm{c})$ and differences in the secondary velocity magnitudes $\left(\mathrm{ms}^{-1}\right)(\mathrm{b}, \mathrm{d})$ at the Lizerne-Rhône cross-section $6(a, b)$ and the Grande Eau-Rhône cross-section 3 (c,d) confluences. view is looking downstream.
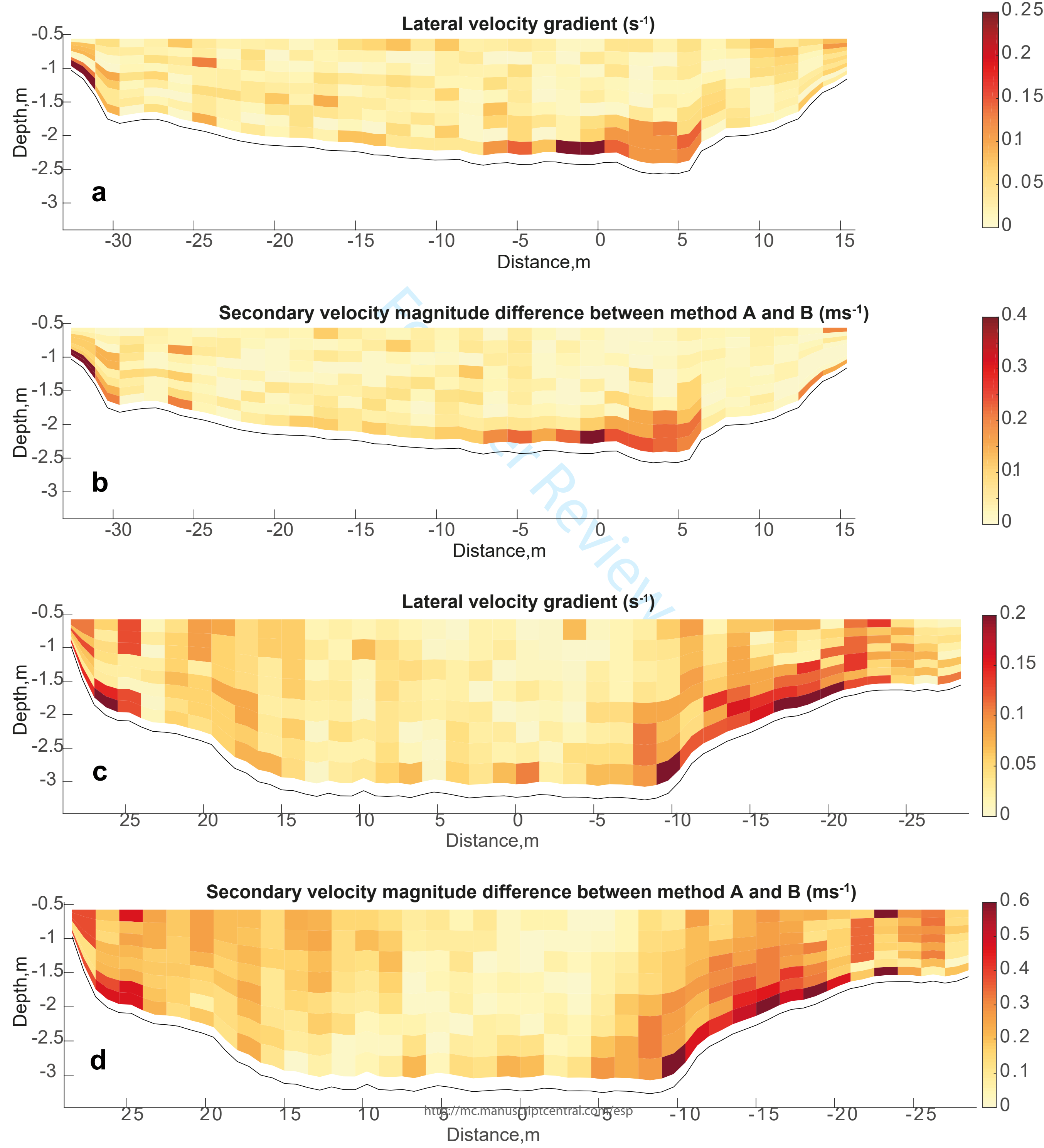
Figure 10: Water column and mesh cells for cross section 9 in Figure 3a at the Lizerne-Rhône confluence, in which standard deviation of the esti-mated velocities have been calculated. view is looking downstream.

11

\section{Lateral velocity gradient $\left(\mathbf{s}^{1}\right)$}

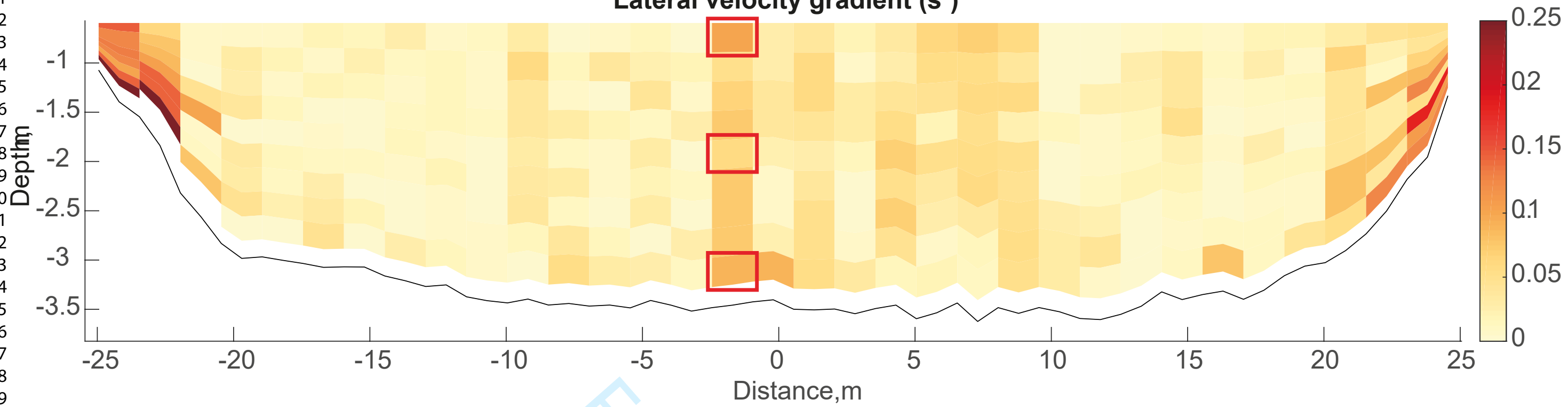


Figure 11: Standard deviation of the velocity estimated using methods A and B for 16 repeats at the Lizerne-Rhône confluence cross-section 9
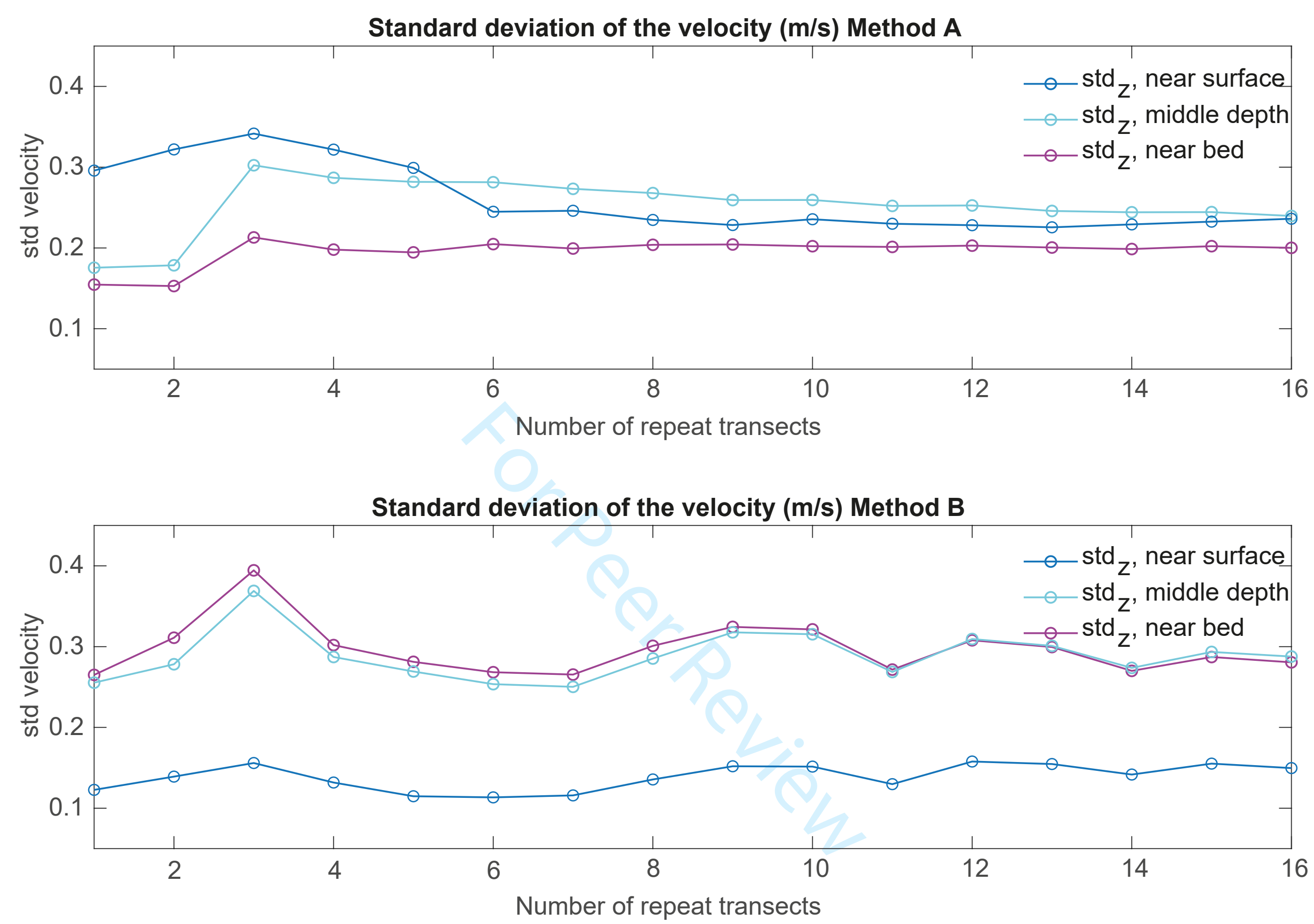
Figure 12: Error distributions related to GPS for a) Lizerne-Rhône confluence (cross-section 6) and c) Grande Eau- Rhône confluence (cross-section 3), and sensors accuracies for b) Lizerne-Rhône confluence (cross-section 6) and d) Grande Eau-Rhône confluence (cross-section 3), in estimating the secondary velocities using method $A$. view is looking downstream.

Magnitude of error

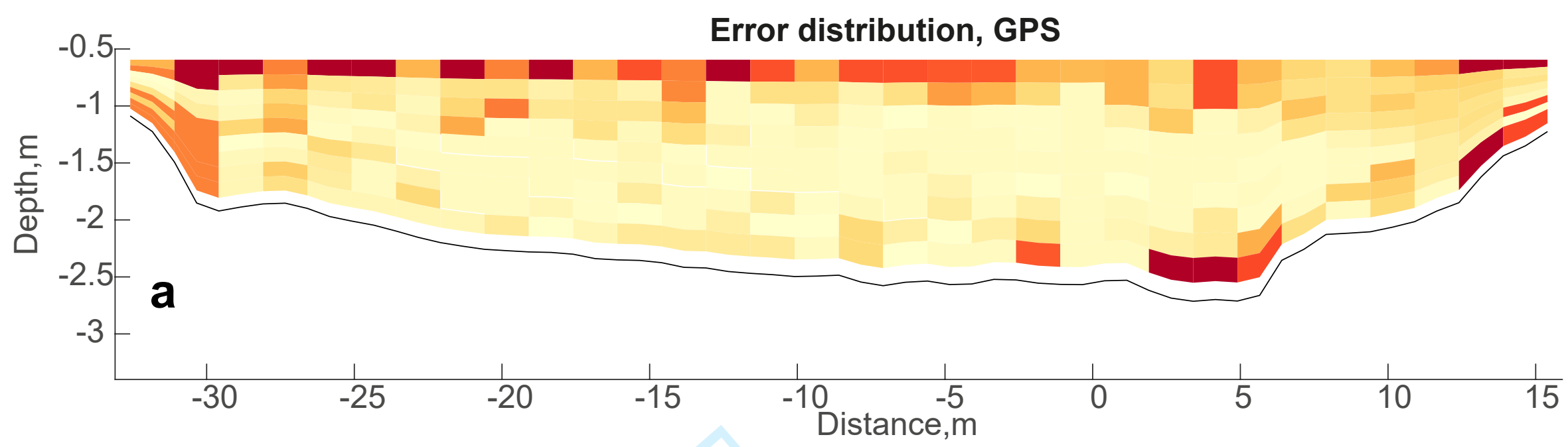
$\mathrm{ms}^{-1}$

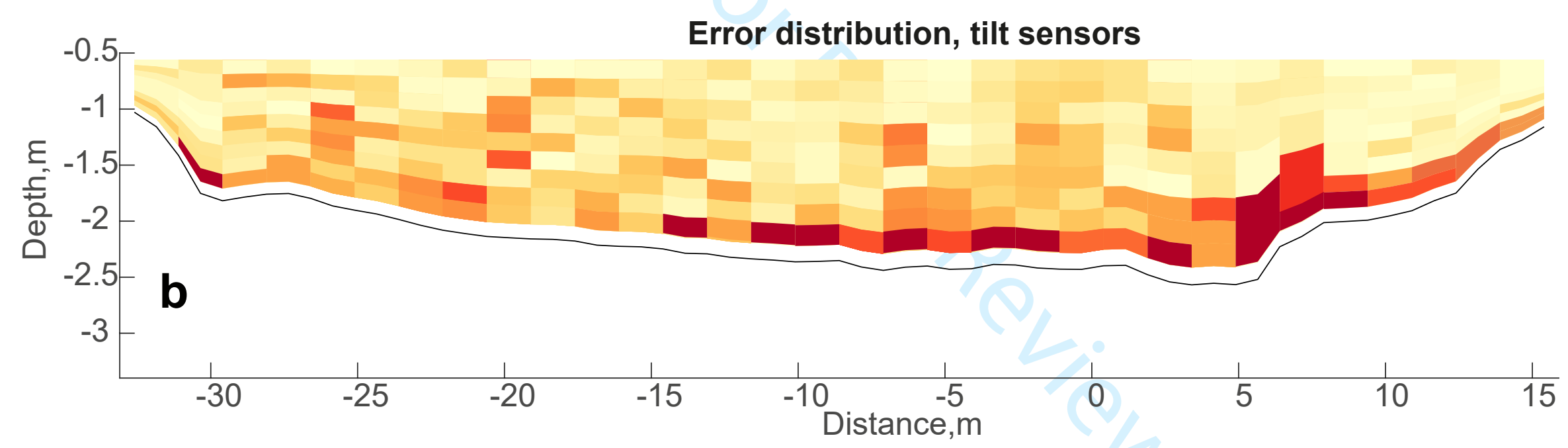

Magnitude of error $\mathrm{ms}^{-1}$

0.014
0.012
0.01
0.008
0.006
0.004
0.002

Magnitude of error

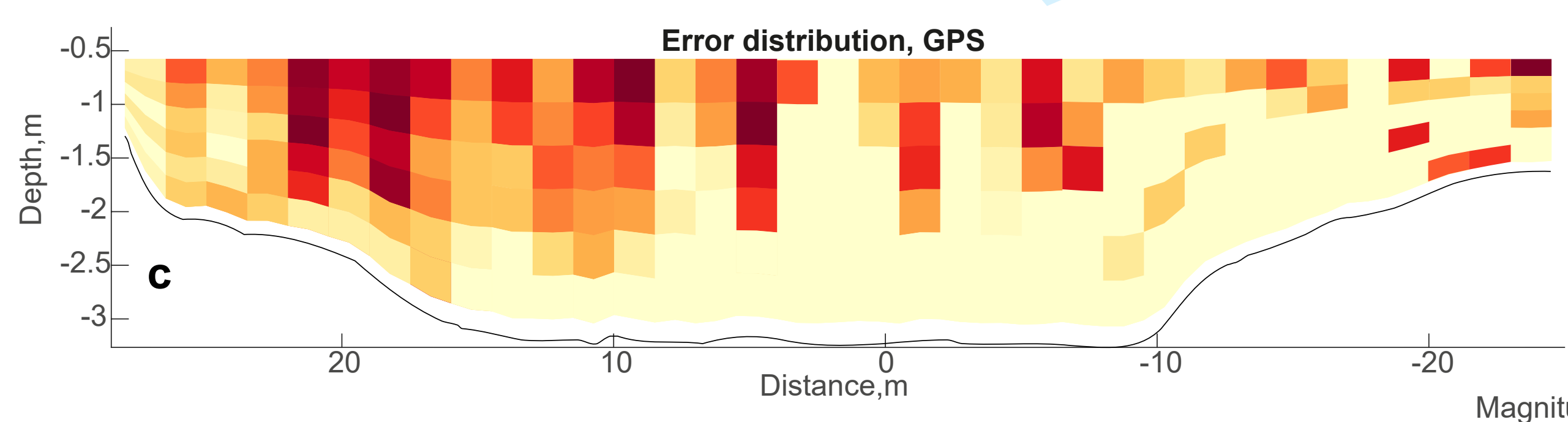
$\mathrm{ms}^{-1}$

0.03
0.025
0.02
0.015
0.01
0.005

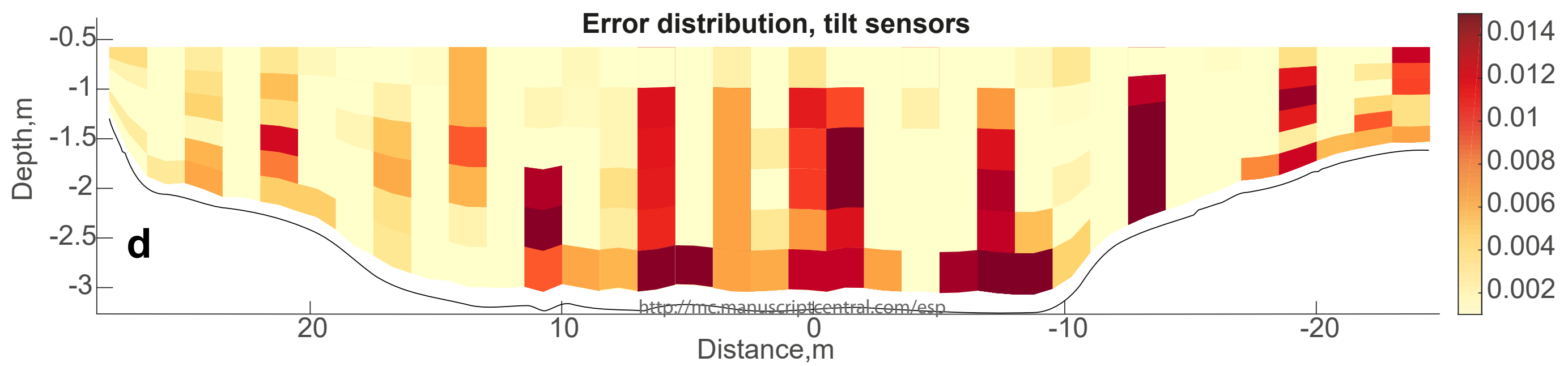


Figure 13: Maximum inhomogeneity allowance $(\mathrm{m})$ using method B for a) Lizerne-Rhône confluence at cross-section 6 and b) Grande EauRhône confluence at cross-section 3. view is looking downstream.

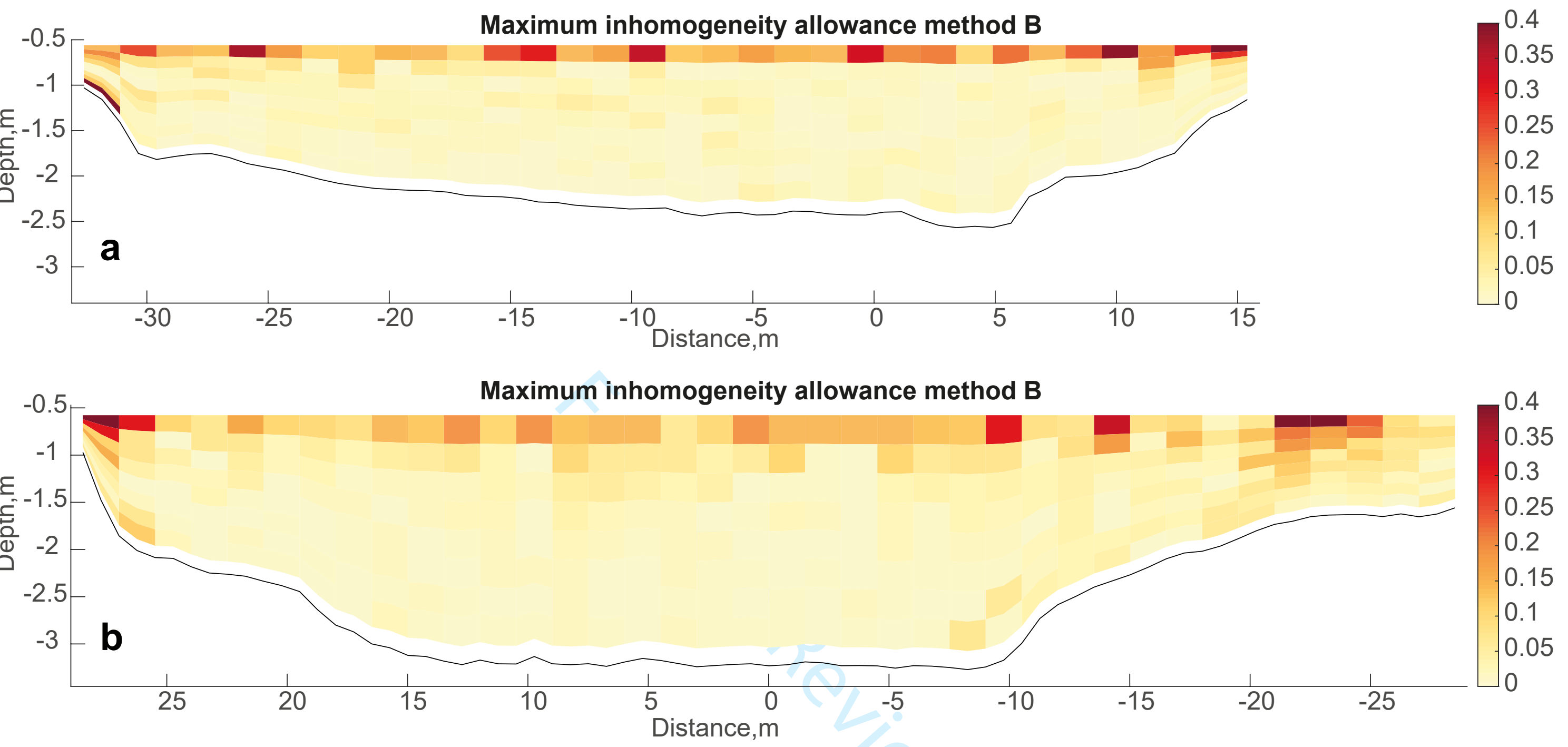


Figure 14: Primary velocities (contours) with secondary velocity vectors estimated using method A and B at cross sections 4,5 and 7 in Figure $3 a$ at the Lizerne-Rhône confluence. view is looking downstream.

\section{Primary velocities with Secondary flow vectors, Method A}

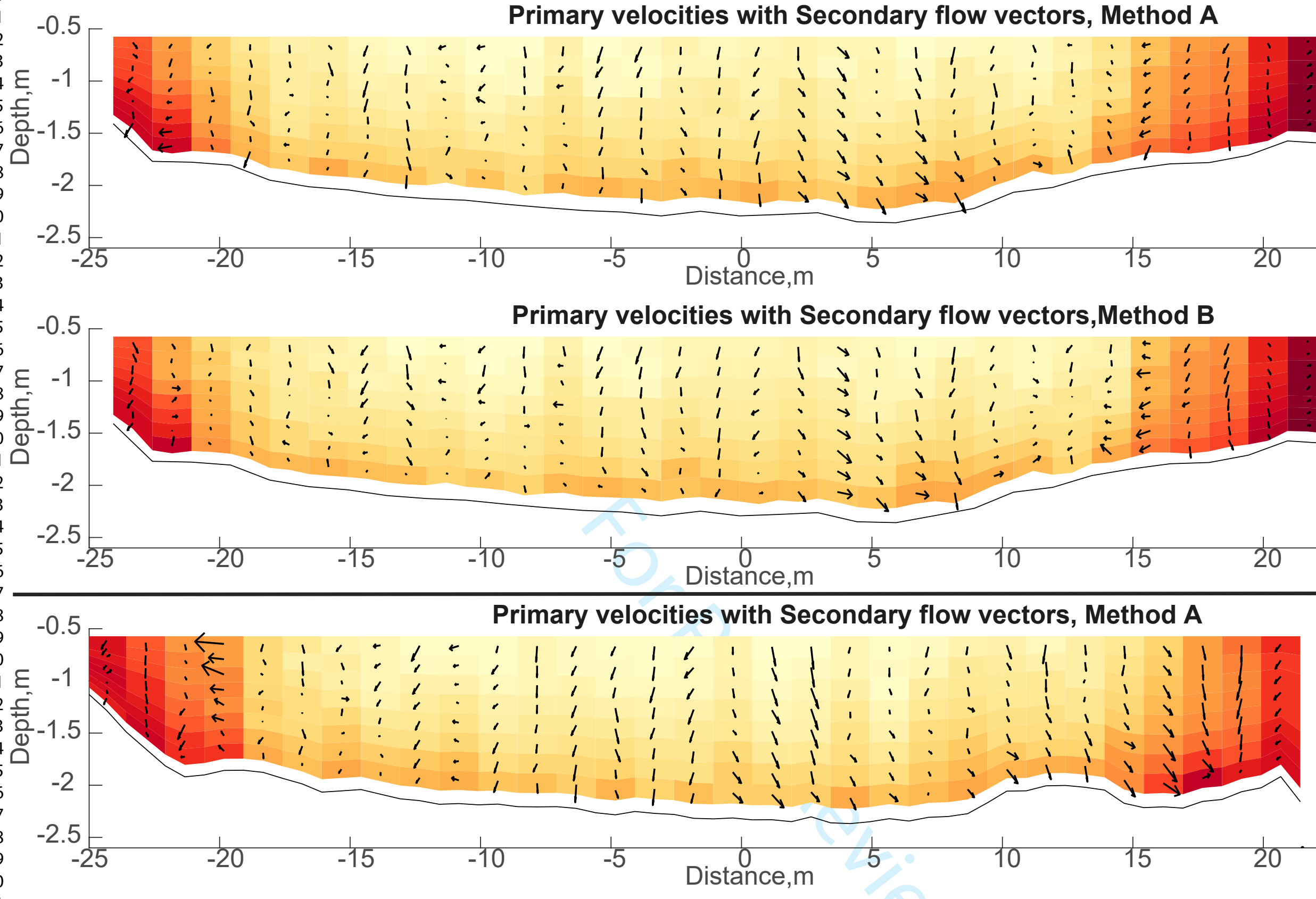

Primary velocities with Secondary flow vectors, Method B

Primary velocities

Primary velocities

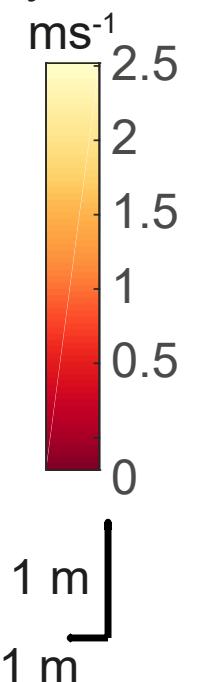

$1 \mathrm{~m}$

4 51 52
53
54
5
5
5
58
5 $-0.5$

\section{$, 1,1,1,1,1,1,1,1$}
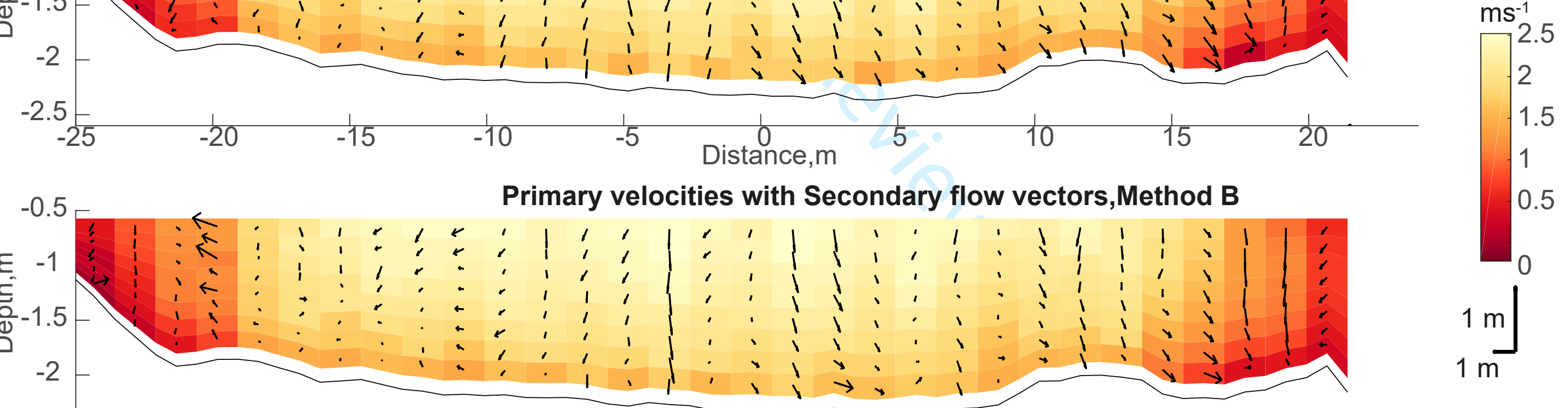

$-2.5$

$-25$

$-20$

$-15$

$-10$

$\stackrel{1}{-5} \quad$ Distance, $m$

10

15

20

$1 \mathrm{~m}$ $1 \mathrm{~m}$

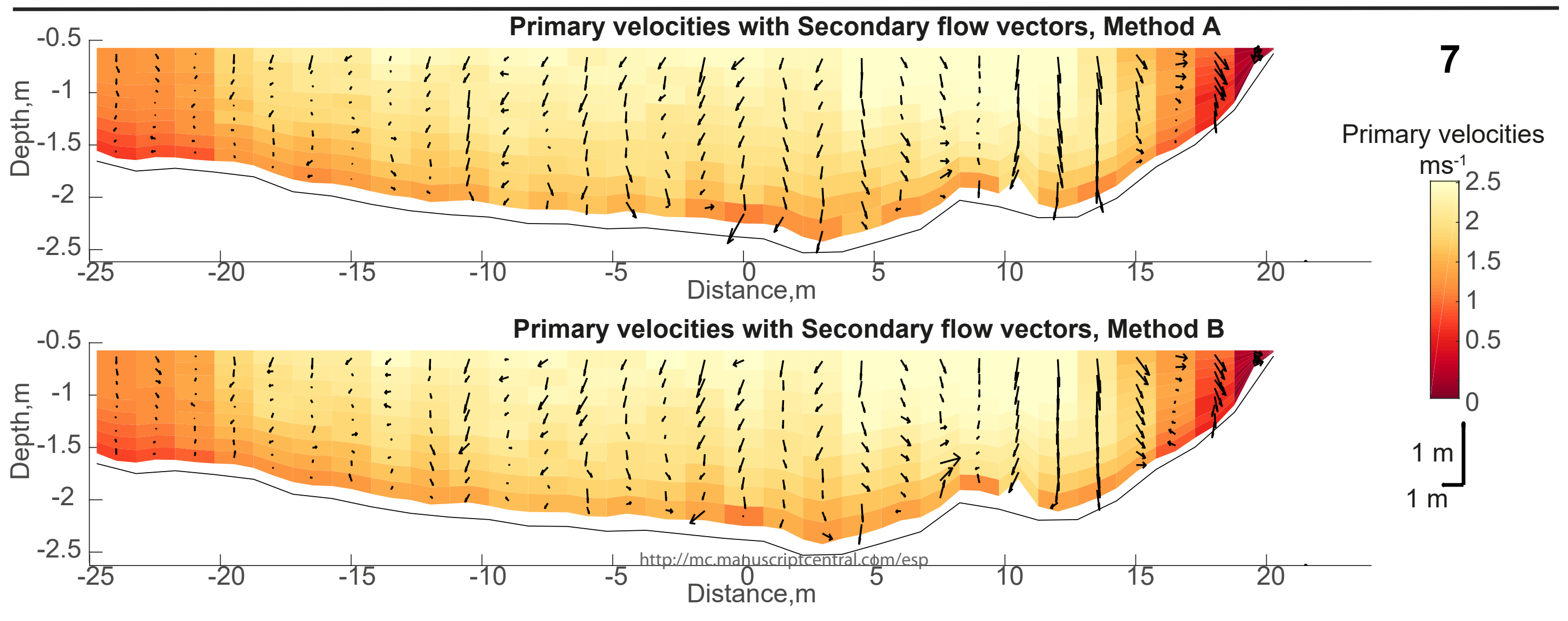


Figure 15: Primary velocities (contours) with secondary velocity vectors estimated using method A and B at cross sections 4,6 and 8 in Figure $3 \mathrm{~b}$ at the Grande Eau-Rhône confluence. view is looking downstream.
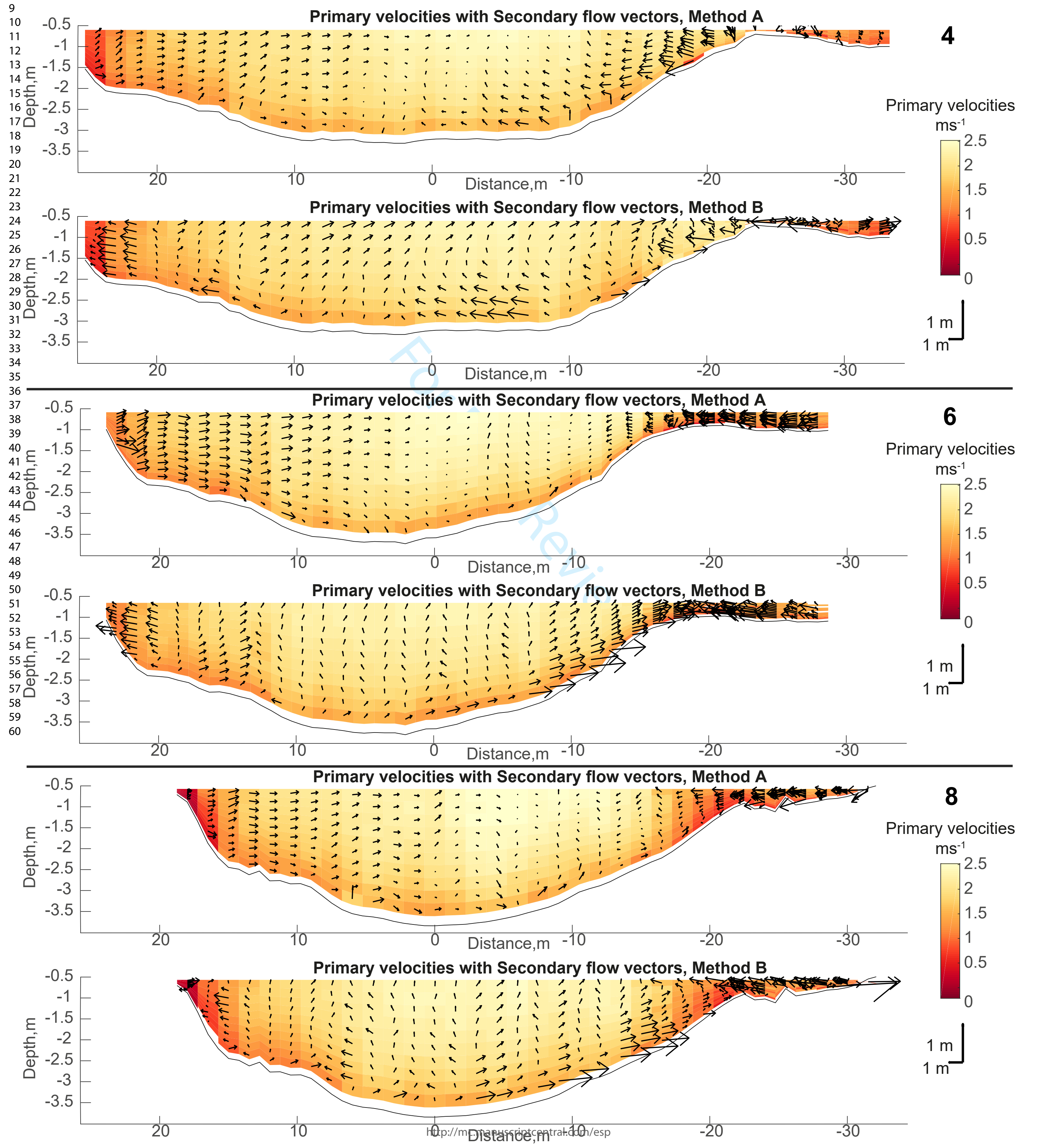
Figure 16: Relationship between lateral velocity gradient, depth and relative differences in secondary velocities for a) the Lizerne-Rhône confluence at cross-section 6 and b) the Grande Eau-Rhône confluence at cross-section 3

a Lateral velocity gradient $\left(\mathrm{s}^{-1}\right)$

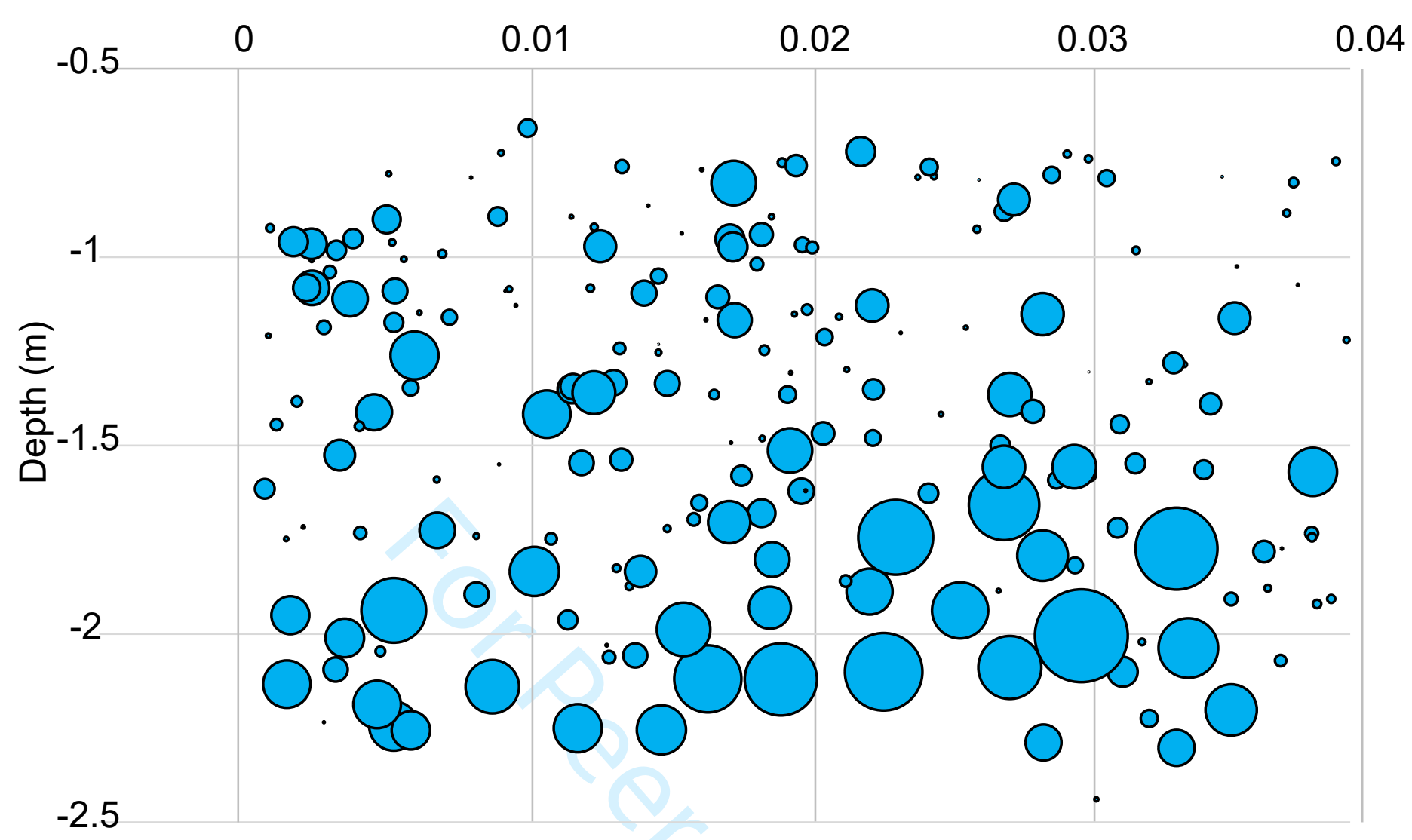

- Relative differences in secondary velocities $\left(\mathrm{ms}^{-1}\right)$

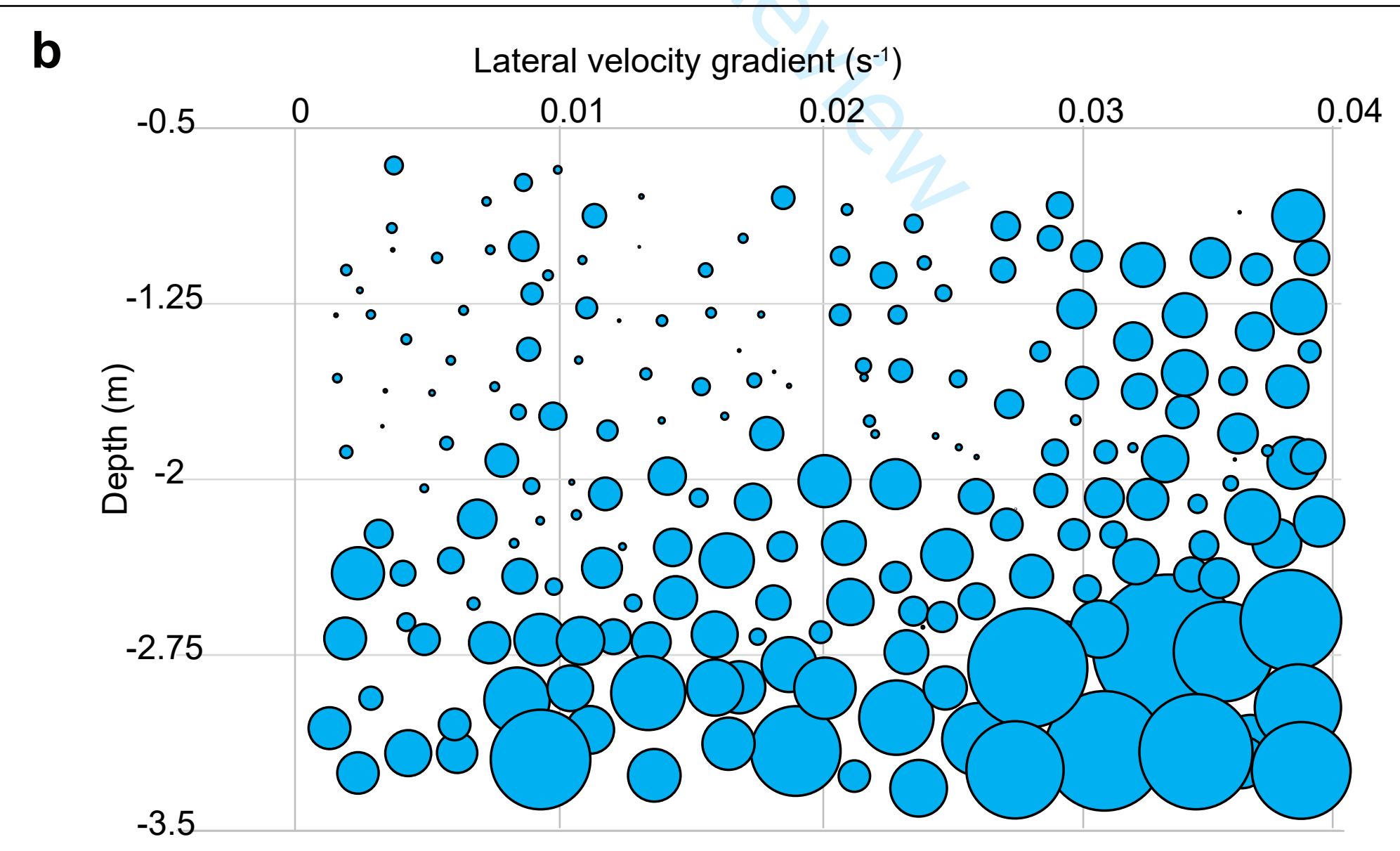

- Relative differences in secondary velocities $\left(\mathrm{ms}^{-1}\right)$ 
1 "For the ESPL special issue: Measuring and numerical modelling of hydro-

2 morphological processes in open-water"

\section{Evaluation of aDcp processing options for secondary flow}

\section{4 identification at river junctions}

5 Gelare Moradi ${ }^{1}$, Bart Vermeulen ${ }^{3}$, Colin D. Rennie ${ }^{2}$, Romain Cardot ${ }^{1}$, Stuart N. Lane ${ }^{1}$

6 1: University of Lausanne - Institute of Earth Surface Dynamics (UNIL - IDYST)

7 Université de Lausanne - IDYST Quartier Mouline - Bâtiment Géopolis - Switzerland

8 2: Civil Engineering Department, University of Ottawa

9 3: Water Engineering and Management Department, University of Twente, the

10 Netherlands

\section{Abstract}

12 Secondary circulation in river confluences results in a spatial and temporal variation of

13 fluid motion and a relatively high level of morphodynamic change. Acoustic Doppler

14 current profiler (aDcp) vessel-mounted flow measurements are now commonly used to 15 quantify such circulation in shallow water fluvial environments. It is well established that

16 such quantification using vessel-mounted aDcps requires repeated survey of the same 17 cross-section. However, less attention has been given to how to process these data. Most 18 aDcp data processing techniques make the assumption of homogeneity between the 19 measured radial components of velocity. As acoustic beams diverge with distance from 20 the aDcp probe, the volume of the flow that must be assumed to be homogeneous 21 between the beams increases. In the presence of secondary circulation cells, and where 22 there are strong rates of shear in the flow, the homogeneity assumption may not apply, 
23 especially deeper in the water column and close to the bed. To reduce dependence on 24 this assumption, we apply a newly-established method to aDcp data obtained for two 25 medium-sized ( $\sim 60-80 \mathrm{~m}$ wide) gravel-bed river confluences and compare the results with 26 those from more conventional data processing approaches. The comparsion confirms that 27 in the presence of strong shear our method produces different results to more 28 conventional approaches. In the absence of a third set of fully independent data, we 29 cannot demonstrate conclusively which method is best, but our method involves less 30 averaging and so in the presence of strong shear is likely to be more reliable. We conclude 31 that it is wise to apply both our method and more conventional methods to identify where 32 data analysis might be impacted upon by strong shear and where inferences of secondary 33 circulation may need to be made more cautiously.

\section{Keywords}

37 Acoustic Doppler current profiler

38 Secondary circulation

39 River confluences

40 River junctions

41 Introduction

42 Acoustic Doppler current profilers (aDcps) are now used widely to measure river flow in 43 three-dimensions, notably for the quantification of secondary flows. Applications have 
44 been made to river bedforms (e.g., Parsons et al., 2005; Kostaschuk et al., 2009; Shugar 45 et al., 2010), bends (e.g., Dinehart and Burau, 2005; Kasvi et al., 2013; Vermeulen et al., 46 2014a, 2015; Engel and Rhoads, 2016; Knox and Latrubesse, 2016; Kasvi et al., 2017; 47 Lotsari et al., 2017; Parsapour-Moghaddam and Rennie, 2018), junctions (e.g., Parsons 48 et al., 2007; Lane et al., 2008; Szupiany et al., 2009; Riley and Rhoads, 2012; Riley et al., 49 2015; Gualtieri et al., 2017), bifurcations (e.g., Parsons et al., 2007; Szupiany et al., 50 2012), canyons (e.g., Alvarez et al., 2017; Tomas et al., 2018; Venditti et al., 2014), deltas 51 (e.g., Czuba et al., 2011) and gravity currents (e.g., Garcia et al., 2007; Garcia et al., 52 2012). Research has also shown the need to make repeat section measurements (e.g., 53 Szupiany et al., 2007; Jackson et al., 2008) and also to process these data carefully, 54 (Muste et al., 2004; Rennie and Church 2010; Tsubaki et al., 2012; Parsons et al., 2013; 55 Petrie et al., 2013). Such processing must take into account positioning (Rennie and 56 Rainville, 2006) and orientation (Zhao et al., 2014) errors, and the treatment of repeat 57 section measurements (e.g., Szupiany et al., 2007; Jackson et al., 2008).

58 This paper is concerned with recent observations regarding the inference of secondary 59 flows from aDcp data and concerns regarding the assumption that flow is homogenous in 60 the fluid volumes defined by the acoustic beams emitted from an aDcp and used to 61 calculate any one point estimate (Vermeulen et al., 2014b). Acoustic beams are reflected 62 by suspended particles, which, if moving, cause a Doppler shift in beam frequency, which 63 is then detected at the sensor. This shift is directional so each beam measures the radial 64 velocity, which is the velocity of particle motion parallel to the acoustic path. This can be 65 assumed to be the flow velocity if the particle motion is identical to fluid motion. In order 66 to resolve flow in more than one direction, aDcps require at least three acoustic beams to 
67 estimate three Cartesian components of velocity. The radial velocities originating from the

68 beams are traditionally analyzed for a single measurement cycle at a single depth at a

69 time (Vermeulen et al., 2014b). The velocity then applies to the volume of fluid defined by

70 the beams at each depth. Flow within this volume is assumed to be homogeneous.

71 However, as the beams spread from the sensor, depth bins increase in horizontal size

72 (Rennie et al., 2002). This means that: (1) bins further from the sensor are likely to produce

73 less reliable velocities because the bin size is greater and the flow within bins is more

74 likely to be heterogeneous (Gunawan et al., 2011); and (2), even in smaller bins, velocities

75 may be less reliable in zones of strong shear where also the within-bin flow is less likely

76 to be homogeneous. In a river where measurements are made throughout the flow depth,

77 the maximum shear may be close to the bed, where the beam divergence may also be 78 greatest.

79 One solution to this problem accounts for first order shear within the flow volume (e.g. 80 Marsden and Ingram, 2004) through a Taylor expansion of the coordinate transform used

81 to determine the Cartesian velocity components. Under this solution, flow is allowed to 82 vary linearly within the bin, but the bin's volume becomes potentially larger with distance 83 from the sensor. Vermeulen et al. (2014b) developed and tested a second solution. As 84 explained in detail below, multiple radial (beam) velocity measurements within a single bin 85 are put through a Cartesian transform to obtain a localized within-bin three-dimensional 86 velocity. This method strongly reduces the volume over which homogeneity should be 87 assumed and Vermeulen et al. (2014b) found that this significantly impacted 88 interpretations of secondary velocities in the presence of strong shear. In this paper, we 89 seek to quantify the effects of this method for the measurement of secondary flow in two 
90 medium-sized river junctions (c. $60-80 \mathrm{~m}$ post-junction channel width). River junctions are 91 associated with very strong shear (e.g. Best and Roy, 1991; Biron et al., 1993, 1996a, 92 1996b; Sukhodolov and Rhoads, 2001; Rhoads and Sukhodolov, 2004, 2008; Konsoer 93 and Rhoads, 2014; Sukhodolov et al., 2017), as well as well-developed secondary 94 circulation (e.g. Ashmore et al., 1992; Rhoads and Kenworthy, 1995, 1998; Rhoads and 95 Sukhodolov et al., 2001; Lane et al., 2008; Riley and Rhoads, 2012; Riley et al., 2015). 96 Thus, understanding how to process effectively the aDcp data used to describe them is of 97 paramount importance.

\section{Methods for estimating Cartesian velocity components from aDcp data}

99 In this section, we describe the two different methodological approaches used in this study 100 to estimate Cartesian velocity components: (1) Method A, the Vermeulen et al., (2014b) 101 method; and (2) Method B, the conventional method. Common to all methods is the 102 assumption that data are available from repeat measurement of the same cross-section, 103 as has been shown to be critical for obtaining reliable estimates of secondary circulation 104 from aDcp data (Szupiany et al., 2007), particularly when single transect measurements 105 are not close enough together.

\section{Method A: based on Vermeulen et al., (2014b)}

107 Application of the Vermeulen et al. (2014b) method requires mapping of radial beam 108 velocity data onto a predefined mesh. This mesh requires both a bottom topography or 109 bathymetric model, and an upper limit just below the water surface. As the measurements 110 were made using several repeat transects for each cross section, the first step is to define 111 a mean cross section for each set of individual transects (boat tracks). The second step 
112 is to define a grid mesh for this mean cross section. Third, all measured beam velocities

113 are projected on to this cross section mesh. Finally, the beam velocities within each mesh

114 cell are then used to resolve a Cartesian velocity for the mesh cell. Errors that influence

115 these steps can be estimated.

116 The first step is estimation of the mesh extremes, both the lower boundary or bathymetry

117 model and the upper boundary near the water surface. To generate the bathymetry model

118 we use depth soundings collected with the aDcp. We recognize that each beam may

119 register a different distance of the stream bed from the sounder, especially as we are

120 dealing with bathymetrically irregular cross-sections. Specifically, for each bottom track

121 sounding within each transect, we use the UTM coordinates obtained with a coupled

122 differential GPS (dGPS), the range of each bottom track beam return, and the instrument

123 tilt to estimate the bed elevation and horizontal position of each beam impingement point

124 on the bed. These bed positions are combined together to identify an initial mean transect.

125 Provided a point is within a certain distance from the initial mean cross-section, LOWESS

126 interpolation (Appendix A) is applied, which has the effect of defining a bathymetric model

127 that gives most weight to points that appear to be closer to the cross-section. It is important

128 to note that this mean transect is not necessarily orthogonal to the primary flow direction

129 and so will not yield true primary and secondary flow estimates without further correction.

130 We address this below.

131 Once the initial bathymetric model is defined, we estimate a unique vector using the initial

132 mean transect; that is the principal direction of the scatter cloud of all $x$ and $y$ UTM 133 positions at the bed. This unique vector points in the direction of the largest eigenvector 134 of the covariance matrix of all UTM positions $(t)$. We then calculate the mean UTM position 
$135\left(p_{\text {mean }}\right)$ for each set of individual transects and the difference between each measured 136 beam position $\left(p_{b}\right)$ and the mean position. The dot product of these obtained values and 137 the unique vector is then used to define the projection of each UTM position in the direction 138 of the unique vector. To identify the final mean cross section, we sum up all individual 139 projected vectors and obtain the best fit to all available data (Figure 1).

140 To define the upper boundary of the mesh, we estimate the elevation of the water surface.

141 As there is a blanking distance at the surface of the water during the measurement, we

142 then remove this blanking distance, taken as $0.30 \mathrm{~m}$. Thus, the mesh has also a blanking

143 distance and the upper part of the cross-section is, strictly, the upper limit of available 144 data, not the water surface.

145 "Figure1"

146 The second step uses the defined bathymetric model and available velocity bins within 147 the measured area (not influenced by side lobes, and below the blanking distance) to 148 define a cross-section mesh. The side-lobe interference is caused by the striking of the 149 channel bed by side-lobe energy from each of the acoustic beams. This side-lobe energy 150 has strong reflections from the bed, which result in echoes that overwhelm the signal from 151 scatters near the bed. The thickness of the side-lobe layer is typically $6-7 \%$ of the 152 measured depth (Morlock, 1996).

153 To generate the mesh, the cross section is initially subdivided into vertical slices with equal 154 widths $(\Delta n)$. For each slice, the simplest definition of mesh cell thicknesses $(\Delta z)$ divides 155 each vertical equally. These verticals are converted to non-dimensional $\sigma$ coordinates 156 using following equation: 
$157 \sigma=1-\left(\frac{p_{v} \cdot \boldsymbol{k}-\eta}{p_{b} \cdot \boldsymbol{k}-\eta}\right) \quad$ (Vermeulen et al. 2014b)

158 where $p_{v}$ stands for velocity measurement positions $(m), p_{b}$ is the corresponding bed 159 position $(\mathrm{m})$ that is found using velocity measurement horizontal positions and applying 160 the bathymetric model, $\boldsymbol{k}$ is the upward pointing unit vector and $\eta$ are the water surface 161 fluctuations around the mean water level at which $z=0$.

162 However, because of beam spreading and differences in the distance of the sounder from 163 the bed, which varies with position of the sounder, this tends to produce a highly 164 heterogeneous number of measurements in each cell within the mesh. The alternative, 165 adopted here, is to allow mesh cell thickness to vary through the water column such that 166 there is a roughly equal number of beam velocities contributing to each mesh cell (see 167 Figure 2 for a typical distribution).

168 As the river bed form is varying, to follow its shape, each mesh cell is considered to be a 169 cuboid with 6 edges, two on the left side, two in the middle and two on the right side. To 170 define these edges, the first step is to define the middle point of each mesh cell. Once 171 defined, by calculating the slope for each half part of the mesh cell, edges can be obtained. 172 The mesh cell faces are then calculated on the basis of adjacent verticals and the mesh 173 cell upper and lower boundaries.

174 To identify the beams that contribute to each mesh cell, an index for each beam velocity 175 is defined, which shows its associated mesh cell, using the projection of each radial 176 velocity onto the estimated mean cross section (Figure 2).

177 "Figure 2" 
178 In the third step, the radial velocities for each beam (b) that contribute to each mesh cell 179 (the $\mathrm{N}$ beam velocities) have to be transformed into Cartesian velocities $\left(\mathrm{v}_{\mathrm{x}}, \mathrm{v}_{\mathrm{y}}\right.$ and $\left.\mathrm{v}_{\mathrm{z}}\right)$ 180 using:

$181 \quad\left(\begin{array}{c}b_{1} \\ \vdots \\ b_{N}\end{array}\right)=\left(\begin{array}{c}q_{1} \\ \vdots \\ q_{N}\end{array}\right) \cdot\left(\begin{array}{c}v_{x} \\ v_{y} \\ v_{z}\end{array}\right) \leftrightarrow b=Q \cdot u$

182 where $\mathbf{q}$ is a unit vector which describes the direction of the acoustic beam.

183 To obtain the raw beam velocities, we use matrix transformations obtained from the raw 184 data to transform measured velocities in $\mathrm{XYZ}$ coordinates into beam velocities. The 185 Vermeulen et al., (2014b) method includes in the transformations an explicit treatment of 186 the random errors due to internal and external factors and the bias (systematic errors) 187 caused by the measurement system and the nature of river flow (Tsubaki et al., 2012). 188 Random errors include those that come from sampling a time-varying flow in the presence 189 of strong gradients and represent a form of aliasing. By adding a combined term of errors $190 \varepsilon,(2)$ becomes:

$191 \quad b=Q u+\varepsilon$

192 A least squares solution is fitted to (3) that minimizes the sum of the square of the errors. 193 The optimal estimation $(\hat{\mathbf{u}})$ for $(\mathbf{u})$ is then given by the normal equation:

$194 \hat{\mathbf{u}}=Q^{+} \mathbf{b}+\varepsilon$

195 where $\mathrm{Q}^{+}$can be defined as:

$196 Q^{+}=\left(Q^{\top} Q\right)^{-1} Q^{\top}$ 
197 To solve three Cartesian velocity components, we need at least three equations. Each 198 beam measurement in a mesh cell adds an equation. Where enough beam velocities are 199 collected in a mesh cell and the equations are different from each other (beam velocities 200 are measured from different directions), the velocity can be estimated. To check whether 201 this is the case, the matrix describing the system of equations can be analyzed. In the 202 processing we use the rank which indicates how many unknowns can be solved from the 203 system of equations. When the rank is three, the three Cartesian velocities can be solved. 204 Where the rank of the matrix is one or two, the system cannot be solved. Where the 205 system of equations is overdetermined, the obtained solution is a matrix with more 206 equations (rows) than unknowns (columns). The velocity can be solved using the 207 generalized inverse of the matrix and in such a way that the sum of squared errors is 208 minimized. As this combined term of errors also contains information about the turbulence 209 and accuracy of the measurements, we can obtain the covariance matrix of the velocity 210 components:

$211 \hat{\varepsilon}=\mathbf{b}-Q \hat{u}$

$212 \operatorname{var}(\hat{\mathbf{u}})=\frac{\hat{\varepsilon}^{\top} \hat{\varepsilon}\left(Q^{\top} Q\right)^{-1}}{\mathrm{~N}-3}$

213 and the variance of the velocity across the section can be then estimated as:

$214 \operatorname{var}(\mathbf{u})=\frac{\operatorname{var}(\hat{\mathbf{u}})}{\mathrm{N}}$

$215(8)$

216 Method B: the standard aDcp method

217 As the Doppler shift is directional, it can only measure radial velocities. With the standard 218 method, to determine Cartesian velocity components, radial velocities then have to be 
219 resolved into three orthogonal velocity vectors. To do so, at least three beam velocities 220 pointed in known directions are required. Also, because the beams are measuring 221 different water profiles along their individual slant ranges, the assumption of horizontal 222 homogeneity must be taken into account. Hence, in the standard method, the three 223 dimensional velocity for each depth bin for each ping can be solved for a typical four-beam 224 system using the following equations (Mueller and Wagner, 2009):

$225 \quad V_{x}=\frac{\left(b_{3}-b_{1}\right)}{\sqrt{2} \sin \theta}$

$226 \quad V_{y}=\frac{\left(b_{4}-b_{2}\right)}{\sqrt{2} \sin \theta}$

$227 \quad V_{z}=\frac{-\left(b_{1}+b_{3}\right)}{(2 \cos \theta)}=\frac{-\left(b_{2}+b_{4}\right)}{(2 \cos \theta)}$

$228(11)$

229 where $V_{y}$ is the cross stream velocity assuming beam 3 is pointed upstream, $V_{x}$ is the 230 streamwise velocity, $V_{z}$ is the vertical velocity, $b_{1}, b_{2}, b_{3}$ and $b_{4}$ are the radial velocities 231 measured in beams $1,2,3$ and 4 respectively and $\theta$ is the tilt angle of the beams referenced 232 to vertical. These data should then be corrected for pitch and roll angles, obtained from 233 the internal inclinometer and the heading angle from the internal compass. Velocity 234 outputs are already corrected for ship velocities.

235 To compare results obtained using Method B with those of Method A, we use the same 236 mean cross section built for Method A, as well as the same bathymetric model and the 237 same mesh. Each measured velocity vector is assigned to the appropriate mesh cell by 238 projecting its 3D position (horizontal position and depth) onto the mean cross section 
239 mesh. We then average $x, y$, and $z$ components of all velocities measured within a mesh 240 cell to obtain the mean velocity vector for the mesh cell.

\section{Methodology}

242 This paper is motivated by the need to acquire three-dimensional data from junctions of 243 tributaries with a main river stem, here the River Rhône, western Switzerland, and so the 244 need to identify methods for reliably obtaining Cartesian velocities from aDcp data. The 245 Rhône tributaries typically have very high bedload transport rates for short periods of time, 246 leading to the formation of very large tributary mouth bars downstream of their junctions 247 with the main river. These bars are maintained for weeks or months such that at lower 248 tributary flow, with negligible sediment supply, there is a legacy effect of previous high 249 momentum tributary events upon junction morphology and secondary flow formation.

250 For this paper, we used a specially-designed rope and pulley system to collect aDcp data 251 from the junction of two tributaries with the Rhône (Figure 3).

252 "Figure 3"

253 The Lizerne is a Rhône tributary of almost $20 \mathrm{~km}$ length that flows south-westward from 254 the western slopes of the Tête Noire $(2451 \mathrm{~m})$ or La Fava $(2612 \mathrm{~m})$, in the Bernese Alps. 255 This river is heavily regulated for hydropower with sediment extracted upstream of the 256 junction. As a result, there is negligible sediment supply and no evidence of point bar 257 formation. It reaches the Rhône between Ardon and Vétroz, forming a $90^{\circ}$ junction angle 258 and it has a bed that is nearly concordant with the Rhône. 
259 The Grande Eau is a second tributary of the Rhône River which has a length of $26 \mathrm{~km}$ and 260 takes its source on the Vaud side of the Les Diablerets and flows into the Rhône River 261 with a $70^{\circ}$ confluence angle, near Aigle. The Grande Eau bed is $1.5 \mathrm{~m}$ higher than the 262 Rhône such that it is markedly discordant.

263 In this section, we: (1) describe the aDcp used to collect data; (2) describe how the aDcp 264 was deployed; and (3) outline the analytical approaches used to interpret the results from 265 the different methods. Although the method is valid for any aDcp that has an onboard 266 compass and potential for differential GPS positioning, as is standard with most aDcps, 267 we use a Sontek M9 aDcp in this study.

\section{The Sontek M9 aDcp}

269 The SonTek M9 aDcp is a nine-transducer system with three acoustic frequencies, 270 configured as two sets of four profiling beams $(3 \mathrm{MHz}$ and $1 \mathrm{MHz}$ transducers in Janus 271 configurations) and one vertical beam ( $0.5 \mathrm{MHz}$ Echo sounder) for depth measurements 272 (SonTek YSI, 2010). It uses these two sets of four beams to provide raw radial velocity 273 samples. These beams are equally spaced at $90^{\circ}$ azimuth angles and are projected at an 274 angle $\theta$ of $25^{\circ}$ off the vertical axis (SonTek YSI, 2000). For the standard configuration, 275 the four beams encompass a sampling diameter of $93 \%$ of the distance from the aDcp 276 (7\% of side-lobe) (SonTek YSI, 2000).

277 The output velocities from the SonTek M9 Riversurveyor are either in Cartesian 278 coordinates $(X Y Z)$ that are relative to sensor orientation or in Earth coordinates (ENU) for 279 a SonTek system with compass and tilt sensors. These raw velocity data in Earth 280 coordinates or XYZ coordinates are already corrected for the ship motion. To apply 
281 Method A to Sontek output data, as this method is based on radial velocities, it is 282 necessary to transform these output velocities to radial velocities. To do so, we add ship 283 velocities to these output velocities and then apply the inverses of the instrument's matrix 284 coordinate transformations (obtained from MATLAB files output by the SonTek data 285 collection software RiverSurveyor). As the survey is being undertaken using a moving 286 vessel, these radial velocities then have to be corrected again for the boat velocity. There 287 are two key methods for doing this. The first uses the bottom tracking to measure the boat 288 velocity relative to the river bed, under the assumption that the latter is stationary (i.e. 289 there is no bedload transport). The second tracks the boat position using differential GPS 290 (dGPS, e.g. Zhao et al., 2014). In this study, we corrected all raw beam velocities for ship 291 velocities, using dGPS as we could not exclude the possibility of there being bedload 292 transport.

293 To apply Method B in this study, we use the raw velocity data in Earth coordinates and 294 we correct it for pitch and roll angles, obtained from internal inclinometer and heading 295 angle data for the internal compass. For SonTek M9 aDcps, pitch is a y-axis rotation and 296 roll an $\mathrm{x}$-axis rotation.

297 Depending on the water depth and velocity, the Sontek M9 firmware changes the acoustic 298 operating frequency and the water profiling mode on-the-fly, thus the number of sampled 299 points in the vertical varies automatically from one profile to the next. Specifically, when 300 the water is shallower than $0.75 \mathrm{~m}$ and the maximum velocity is less than $0.4 \mathrm{~ms}^{-1}$, the $301 \mathrm{M} 9$ reports data acquired with a $3 \mathrm{MHz}$ frequency using the pulse coherent mode to obtain 302 a $2 \mathrm{~cm}$ depth measurement resolution. For deeper situations, this frequency changes to 1 $303 \mathrm{MHz}$ pulse coherent pings using a $6 \mathrm{~cm}$ aDcp cell size. If the maximum velocity is greater 304 than $0.4 \mathrm{~ms}^{-1}$ then SmartPulse (i.e., broadband) mode is utilized, with the $3 \mathrm{MHz}$ beams 
305 if depth is less than $5 \mathrm{~m}$ and the $1 \mathrm{MHz}$ beams if depth is greater than $5 \mathrm{~m}$, with the aDcp 306 cell size optimized based on the current water depth. As a result of these on-the-fly 307 changes, each measured profile has a different number of aDcp cells and different aDcp 308 cell sizes. Hence, to correct the aDcp cell size variability, for both methods A and B there 309 is the need to define a cross-sectional mesh and to project the measured velocities to this 310 mesh. For Method A we use the beam velocity vertical positions in a non-dimensionalized 311 coordinate system using equation 1 , within the predefined mesh explained in section 2.1.

\section{Deployment of the Sontek M9 in the river junctions}

313 The survey work was undertaken in two junctions of the Swiss River Rhône, the Lizerne314 Rhône confluence in August 2017 and the Grande Eau-Rhône confluence in May 2018, 315 using a Sontek M9 vessel mounted aDcp and a specially-designed rope-pulley system 316 (Figure 3c). The survey was spatial, monitoring 11 cross-sections from upstream of the 317 junction to its downstream at the Lizerne-Rhône confluence with a Momentum ratio (Mr) 318 of 0.018 (Figure 3a) and 11 cross-sections at the Grande Eau-Rhône confluence with a $319 \mathrm{Mr}$ of 0.022 (Figure $3 \mathrm{~b}$ ). Table 1 shows the general characteristics of these two 320 confluences on the date of the measurements.

\section{1 "Table 1"}

322 As proposed previously by Dinehart and Burau (2005), Szupiany et al. (2007), Gunawan 323 et al. (2011) and Vermeulen et al., (2014b) at least five repeats are required to have a 324 robust estimation of secondary velocities. Hence, in this paper, data are processed for 325 cross-section 6 at the Lizerne-Rhône confluence (Figure 3a), and for cross section 3 at 326 the Grande Eau-Rhône confluence (Figure 3b). Identification of the minimum number of 
327 repeat transects necessary per cross-section was undertaken using cross-section 9 at the

328 Lizerne-Rhône confluence (Figure 3a), which involves 16 repetitions. We noted that after

329 application of Method A, the standard deviation of velocity stabilized with six repetitions, 330 which is the number we adopt for this study.

\section{Bin position error determination}

332 Application of Method A requires estimation of the error terms in (2). The size of the 333 sampling volume in each beam is determined by the size of the bin used. As the SonTek 334 M9 aDcp uses different bin sizes depending on the water track frequency (section 2.1.3), 335 these volumes could vary. Applying Method A might improve the velocity estimation for 336 large measurement volumes at depth, as it does not rely on the homogeneity assumption. 337 But as bins with a small number of velocity measurements will have greater error, this 338 method can estimate velocities with error. Also, if the beam velocity distribution within 339 each mesh cell is not linear, as averaging is made in the middle of each mesh cell, it can 340 introduce error in velocity estimation. Thus, it is necessary to calculate a minimum 341 necessary mesh cell size when applying Method A.

342 Method B is inherently limited by spatial averaging due to the potential use of divergent 343 beams and the associated homogeneity assumption. In other words, one must assume 344 that the velocity is homogeneous over the horizontal domain defined by beam divergence 345 (Eq.12). Method A has the advantage that velocities are recorded within an individual 346 beam depth bin, thus no spatial averaging between beams is required. However, in order 347 for Method A to overcome the uncertainty induced by spatial averaging inherent to Method $348 \mathrm{~B}$, it is essential that the bin location is known explicitly. Error in bin location can be induced 349 by dGPS position and or tilt sensor (pitch and roll) errors. We therefore compare possible 
350 bin position errors using Method A to beam divergence obtained from Method B to indicate 351 when Method A should be advantageous over Method B.

352 Beam divergence is the spatial separation of the beams due to the Janus configuration of 353 the beams with beam angles of $25^{\circ}$. This divergence determines the sampling volume that 354 must be considered homogeneous for Method B and can be calculated using equation 355 12:

$356 x_{b}=2 d \tan \theta$

357 where $d$ is the depth in $m$ and $\theta$ is the beam angle which for a SonTek aDcp is $25^{\circ}$. The 358 aDcp dGPS is used to reference the velocity measurements in space and to estimate the 359 ship velocity. If dGPS is used for ship velocity, this introduces errors in measurement of 360 the absolute water velocity (because ship velocity is subtracted from the water velocity 361 measured in the reference frame of the aDcp). This uncertainty introduces error in velocity 362 calculations.

363 To estimate the errors due to dGPS and the tilt sensors, in this study we assume normally 364 distributed random errors with a standard deviation of $\pm 1^{\circ}$ for tilt sensors, based on 365 manufacturer specifications, and a normally distributed displacement error measured by 366 the dGPS for the dGPS positions (as a function of satellite configuration during 367 measurement), and we apply a Monte Carlo approach which we run 100 times sampling 368 under these uncertainties. Each time we calculate the estimated secondary velocity 369 differences as compared with the original secondary velocities.

370 To be able to reduce the uncertainty due to velocity estimation using Method A compared 371 to Method B, the errors induced in Method A related to GPS uncertainty and tilt sensors 
372 must be less than the errors in Method B due to beam divergence and the homogeneity 373 assumption. Hence, Method A can be used if the error associated with a minimum aDcp 374 cell size is in between the error due to beam divergence and the maximum estimated error 375 due to the GPS and tilt sensors. Otherwise using this method introduces more error in 376 velocity estimations than using Method B.

\section{Data interpretation}

378 Methods A and B, described above, were applied to the Sontek M9 data, to determine 379 Cartesian velocities $\left(v_{x}, v_{y}\right.$ and $\left.v_{z}\right)$. As our interest is in process estimation, here we 380 describe the methods we apply to the Cartesian velocities to estimate processes relevant 381 to junction dynamics. In order to distinguish between primary and secondary components 382 of flow, we need to rotate the initial mean transect. Options for doing this are reviewed in 383 Lane et al. (2000) and we do not assess them here, but rather apply the zero net cross 384 stream discharge definition (Lane et al., 2000). By calculating the mean values of the $x$ 385 and $y$ velocity components ( $U$ and $V$ ), we then calculate the velocity magnitude $(v)$. By 386 rotating these velocity components to the direction of the cross-stream velocity, using the 387 unique vector $(\boldsymbol{\sigma})$, primary velocity vectors $\left(\boldsymbol{v}_{\boldsymbol{p}}\right)$ and secondary velocity vectors $\left(\boldsymbol{v}_{\boldsymbol{s}}\right)$ then 388 can be estimated.

$389 \quad v=\sqrt{U^{2}+V^{2}}$

$390 \quad(13)$

$391 \quad\left(\begin{array}{l}\sigma_{x} \\ \sigma_{y}\end{array}\right)=\left(\begin{array}{l}U \\ V\end{array}\right) / v$

392 where $\sigma_{\mathrm{x}}$ and $\sigma_{\mathrm{y}}$ are sin and cos of the angle between the section angle and east. 
$393 \quad \boldsymbol{v}_{p}=\sigma_{x} \boldsymbol{v}_{x}+\sigma_{y} \boldsymbol{v}_{y}$

$394 \boldsymbol{v}_{s}=-\sigma_{y} \boldsymbol{v}_{x}+\sigma_{x} \boldsymbol{v}_{y}$

395 However, secondary circulation is all flow that is orthogonal to the primary flow and not 396 just horizontal flow; there should be not net secondary flux in a section; and so correction 397 should also consider vertical velocities. Thus, we extend these relationships to include 398 vertical velocities:

$399 \quad\left(\begin{array}{lll}\sigma_{x, 1} & \sigma_{x, 2} & \sigma_{x, 3} \\ \sigma_{y, 1} & \sigma_{y} & \sigma_{y, 3} \\ \sigma_{z, 1} & \sigma_{z, 2} & \sigma_{z, 3}\end{array}\right)=\left(\begin{array}{c}U \\ V \\ W\end{array}\right) / v$

400 where: $U, V$ and $W$ are the mean velocities of $x, y$ and $z$ velocity components, respectively 401 and $v$ is the magnitude of the velocity which can be obtained using:

$402 v=\sqrt{U^{2}+V^{2}+W^{2}}$

$403 \boldsymbol{v}_{p}=\sigma_{x, 1} \boldsymbol{v}_{x}+\sigma_{x, 2} \boldsymbol{v}_{y}+\sigma_{x, 3} \boldsymbol{v}_{z}$

$404 \quad(19)$

$405 \boldsymbol{v}_{s}=\sigma_{y, 1} \boldsymbol{v}_{x}+\sigma_{y, 2} \boldsymbol{v}_{y}+\sigma_{y, 3} \boldsymbol{v}_{z}$

$406 \quad(20)$

$407 \quad \boldsymbol{v}_{v}=\sigma_{z, 1} \boldsymbol{v}_{x}+\sigma_{z, 2} \boldsymbol{v}_{y}+\sigma_{z, 3} \boldsymbol{v}_{z}$

$408 \quad(21)$

409 To estimate velocity gradients, and to correct for weak curvature with the survey method 410 at the edges of each transect line (e.g. Figure 3), all data have been transformed into row 411 and column coordinates ( $\eta$ and $\zeta$ ) using the following transformation: 
$412 \quad\left(\begin{array}{l}\frac{\partial}{\partial n} \\ \frac{\partial}{\partial z}\end{array}\right)=\left(\begin{array}{ll}\frac{\partial \eta}{\partial n} & \frac{\partial \zeta}{\partial n} \\ \frac{\partial \eta}{\partial z} & \frac{\partial \zeta}{\partial z}\end{array}\right)\left(\begin{array}{c}\frac{\partial}{\partial \eta} \\ \frac{\partial}{\partial \zeta}\end{array}\right)$

413 where $n$ and $z$ are horizontal and vertical coordinates on the section plane, respectively

414 (Vermeulen et al., 2014b).

\section{Results}

\section{Primary and secondary velocities}

417 Primary and secondary velocities estimated using methods $A$ and $B$ for the Lizerne-Rhône 418 confluence appear to be similar at cross-section 6 (Figures $4 a$ and $4 b$ ) and the differences 419 in estimated secondary flows are minor. The differences are most pronounced between 42010 and $5 \mathrm{~m}$, in the middle of the main channel.

421 These primary and secondary velocity patterns show higher differences at cross-section 4223 of the confluence of Grande Eau-Rhône (Figures 4c and 4d) despite it having a similar 423 momentum ratio to the Lizerne during measurement. Primary velocities differ significantly 424 between methods A and B: (1) at greater distance from the aDcp because the bins contain 425 larger volumes of water assumed to be homogenous; and (2) at the edges of the cross426 section where there are more beam velocity measurements (contours in Figures $4 \mathrm{c}$ and $4274 d)$. Secondary velocity vectors estimated using Method A indicate flow convergence at 428 the surface and flow descending towards the riverbed throughout the centre of the channel 429 (Figure 4c). This is due to a high degree of bed discordance between the Grande Eau and 430 the Rhône, which increases the penetration of the tributary flow into the main channel over 431 the junction, and which forms a zone of high lateral and vertical shear, on the one hand, 432 and main channel narrowing because of penetration of the tributary mouth bar on the other 
433 hand. The secondary velocity vectors estimated by Method B show a weaker penetration 434 of the tributary flow into the main channel, which results in a reverse flow towards the bank 435 on the tributary side of the channel at the surface of the mixing interface (Figure $4 d$ ). In 436 this case, the core of the secondary circulation is located in the middle of the main channel 437 and closer to the inner bank.

438 "Figure 4"

439 Figure 5 and Figure 6 quantify the differences in primary and secondary velocity patterns 440 estimated using methods $A$ and $B$, for the Lizerne-Rhône confluence. Figures $5 \mathrm{a}$ and $5 \mathrm{c}$ 441 and Figures $6 a$ show that almost $4 \%$ of mesh cells have a relative difference in primary 442 velocities between methods $A$ and $B$ of more than $10 \%$. These differences can exceed $4430.2 \mathrm{~ms}^{-1}$ and so they are relatively small. Velocity differences are more pronounced in 444 estimated secondary velocities, with almost $82 \%$ of mesh cells having a difference of more 445 than $10 \%$, and almost $37 \%$ of mesh cells having a difference of more than $50 \%$ (Figure $4465 b, 5 d$ and $6 b)$.

447 "Figure 5"

448 "Figure 6"

449 At the Grande Eau-Rhône confluence, these differences are greater as compared with 450 those of the Lizerne-Rhône confluence. Figures 7a, 7c and 8a show that these differences 451 for primary velocities exceed $0.4 \mathrm{~ms}^{-1}$ in the zone of high vertical and lateral shear and 452 near the inner bank. Almost $20 \%$ of the mesh cells have a relative difference in primary 453 velocities between methods $A$ and $B$ of more than $10 \%$. The secondary velocity 454 differences are more pronounced between these two methods. Figures $7 \mathrm{~b}$ and $7 \mathrm{~d}$ show 
455 differences with a magnitude of $0.4 \mathrm{~ms}^{-1}$ near the edges and near the bed. Almost all the 456 mesh cells have a difference in estimated secondary velocities between two methods.

457 Figure $8 \mathrm{~b}$ shows that almost $93 \%$ of the mesh cells have a relative difference of $10 \%$ 458 between methods $A$ and $B$. although this value decreases to $55 \%$ for a relative difference 459 of $90 \%$ between these two methods.

460 "Figure 7"

461 "Figure 8"

462 Velocity gradients

463 As Figure 9 shows, there is a strong relationship between lateral gradient in secondary 464 velocities and differences between the secondary velocities estimated using methods $A$ 465 and B for both the Lizerne-Rhône and the Grande Eau-Rhône confluences. This is 466 because a stronger velocity gradient increases the probability that the assumption of flow 467 homogeneity within a bin is likely to fail. Indeed, the marked differences between methods $468 \mathrm{~A}$ and $\mathrm{B}$ at the Grande Eau confluence (Figure 7) are also in a zone of strong lateral shear.

469 "Figure 9"

\section{Number of repeat transects}

471 One way to reduce data fluctuations due to random errors and turbulence, during the 472 measurement using moving vessel aDcps, is to average by using several repeat transects 473 together in one cross section. As each estimated velocity measurement is a single sample 474 in time, adding in a repeat section adds in an additional estimated velocity measurement. 475 Under [8], this should cause the variance to increase, despite the number of 476 measurements used in its estimation increasing, until the point at which there are enough 
477 repeats to capture the effects the range of scales of variation in turbulence impacting the 478 measurement. Then, this variance will become stable. At this stage we can consider the 479 number of repeats as the minimum number required to have a robust estimation of 480 secondary velocity vectors that is to have reached estimates of velocity that are 481 asymptotic on this stable state.

482 Here we apply both methods A and B to the survey of 16 repeats at cross-section 9 in 483 Figure $3 a$ at the Lizerne-Rhône confluence. To allow a reasonable comparison, three 484 mesh cells in the middle of the cross section, and at three different depths (near the 485 surface, middle depth and near the bed) have been chosen (Figure 11). Results show 486 that by using Method $A$, after six repeats, a stable variance of the velocity estimator is 487 obtained at the Lizerne-Rhône confluence (Figure 11a). Many more repeats are needed 488 using Method B (Figure 11b) and this is likely because Method B uses fewer 489 measurements per mesh cell. These results also show a higher standard deviation of the 490 velocity estimation near the surface, using Method A and before achieving the stable 491 situation. This can be explained by the fact that near the surface Method A is more 492 sensitive to errors caused by positioning, while near the bed, hence with distance from 493 the sounder, as the beam spread increases, the improvement obtained using Method $A$ 494 is more pronounced (Figure 11a).

495 "Figure 10"

496 "Figure 11"

497 DGPS and tilt sensor uncertainty analysis

498 As explained above a normally distributed random error has been applied 100 times to 499 both dGPS positioning (by adding a random offset) and tilt sensors (by changing pitch and 
500 roll angles randomly) and the secondary velocities have been estimated using Method A 501 for each perturbed dataset. As Figure 12 shows, the magnitude of errors related to dGPS 502 accuracy are higher than those related to tilt sensor accuracy, for both confluences. These 503 values can reach $\pm 0.03 \mathrm{~ms}^{-1}$ and confirms the earlier finding of Rennie and Rainville 504 (2006) which showed that GPS corrections can have average errors of about $\pm 0.03 \mathrm{~ms}^{-1}$ 505 (Figures 12a and 12c). These magnitudes are also higher near the surface and near the 506 bed for the Lizerne-Rhône confluence (Figure 12a). Near the surface, as there fewer 507 measurements that can contribute to the estimation of aDcp position and tilt, uncertainties 508 in dGPS data will have a greater effect. Near the bed, as the velocity gradient is higher, 509 errors will be greater as well. Figure 12c shows higher magnitudes near the surface at 510 cross-section 3 in Figure 3b for the Grande Eau-Rhône confluence.

511 Errors related to tilt sensor uncertainty are higher where there is a higher velocity gradient.

512 This is related to the fact that within the mesh cells with higher velocity gradients, as the 513 velocity distribution is not linear, and as averaging is made in the middle of the mesh cell, 514 it is more probable that the velocity will be affected by sensor inaccuracies of bin 515 positioning, and so be in error (Figures $12 b$ and 12d).

516 "Figure 12"

517 Homogeneity assumption analysis

518 Figure13 shows the maximum inhomogeneity allowance, using Method B for both case 519 studies. These results are obtained by dividing the velocity gradient obtained from 520 equation 22 by the divergence of the beams from equation 12 . They confirm that, for the 521 homogeneity assumption to be valid and thus error to be minimized using Method B, the 
522 maximum mesh cell size, which can be used is as small as $5 \mathrm{~cm}$ near the bed. Clearly, 523 this is impossible as the configuration of the beams using aDcps always results in beam 524 divergence greater than $5 \mathrm{~cm}$.

525 "Figure 13"

526 Primary and secondary flow patterns

527 In this section, we compared estimated primary and secondary velocities using methods

$528 \quad A$ and $B$ for other cross sections in Figure 3 for both river confluences.

529 Figure 14 shows the results for cross sections 4,5 and 7 (in Figure 3a) at the Lizerne530 Rhône confluence. These cross sections also show similar results in primary and 531 secondary velocity patterns for both methods A and B. Figure15 shows different patterns 532 in primary and secondary velocities estimation using Method A and B for cross sections 5334,6 and 8 in Figure $3 \mathrm{~b}$ at the Grande Eau-Rhône confluence. Method A leads to the 534 identification of a stronger and more coherent tributary penetration at cross-section 4 and 535 weaker upwelling mid-channel, giving the impression of less intense secondary circulation 536 (Figure 15). At section 6, flow towards the true left across the shallow top of the tributary 537 mouth bar is identified and is coherent with Method A. At the channel-scale there is 538 general flow convergence reflecting channel narrowing (Figure 15). When using Method $539 \mathrm{~B}$, these patterns are less coherent and flow is towards the true right in the vicinity of the 540 tributary mouth bar. These patterns are repeated for section 8 (Figure 15).

541 "Figure 14"

542 "Figure 15" 


\section{Discussion}

544 In this paper we used data collected with boat-mounted aDcp technology at two

545 confluences of the Swiss river Rhône, both with similar and very low momentum ratios

$546(0.018,0.022)$ and analysed these using two different methods, $A$ and $B$, to estimate

547 Cartesian velocity components. Method A is based on a methodological approach

548 developed by Vermeulen et al. (2014b). It differs by treating explicitly each individual beam

549 velocity based on its position within a predefined mesh. Results show that this method

550 reduces the volume over which the flow must be assumed to be homogenous (Fig 13). It

551 can, but not necessarily does, result in differences in estimated primary and secondary

552 velocities as compared with the more traditional method ( $B$ in this study), that involves

553 determining velocities by averaging data from the spreading beams. Our results show that

554 these differences are more pronounced in estimated secondary velocities than primary

555 velocities and are higher where there is a greater lateral velocity gradient (Figure 9). The

556 comparison between the two case studies shows that even though both confluences have

557 a very low momentum ratio, as the confluence of the Grande Eau-Rhône has a more

558 complex shear zone, likely due to the effects of bed discordance, and there are more

559 significant differences in the estimation of primary and secondary velocities. This is related

560 to the extent to which spreading of the aDcp measurement beams influences the

561 secondary velocities, particularly in relation to lateral gradients in flow conditions. More

562 standard methods (Method B in this study) are valid if the flow is completely homogenous

563 over the diameter of the fluid column that the beams spread. This diameter varies over

564 depth and is largest near the bed. In the case of the Grande Eau-Rhône confluence where

565 stronger lateral velocity gradients exist in the flow, individual beams will not be measuring 
566 homogenous conditions, particularly near the bed and in the zone of high shear near the 567 inner bank, because the spread of the beams may be greater in diameter than the width 568 of the zone of lateral velocity variation. In this case, as Method A involves less spatial569 averaging than Method B, it may provide more accurate information on the flow behavior, 570 but such a conclusion really needs a third and independent method to confirm it. At the 571 Lizerne-Rhône confluence, even though the momentum ratio is similar to Grande Eau572 Rhône confluence, there is only more localized shear in the flow and a simplified shear 573 zone (Figure 9). In such a situation, using Method B to detect the large scale patterns of 574 secondary flow may be more advantageous, because it involves more spatial averaging.

575 The above discussion suggests that whether or not high rates of later shear influence the 576 potential importance of Method A depends on distance from the aDcp: with more 577 divergence at greater depths, lower levels of lateral shear are likely to be acceptable. 578 Figures $16 \mathrm{a}$ and $16 \mathrm{~b}$ quantifies the relationship between lateral velocity gradient, depth 579 and the magnitude of the relative differences in secondary velocities estimated using 580 methods $A$ and $B$ for the cross-section 6 of the Lizerne-Rhône and cross-section 3 of the 581 Grande Eau-Rhône confluences, respectively. At the Lizerne-Rhône confluence, as the 582 zone of high lateral shear is absent, even though there is a strong relationship between 583 the magnitude of the relative differences in secondary velocities estimated using methods $584 \quad A$ and $B$ and the depth (Figure 16a), their relationship with the lateral velocity gradients is 585 poor. In contrast, for the case of the Grande Eau-Rhône confluence (Figure 16b), where 586 increasing the lateral velocity gradient and depth results in higher relative differences in 587 secondary velocities. Thus, the need to use Method A will depend on the case being used 588 and the extent to which there is lateral shear at greater distances from the aDcp. This is 
589 why whilst it may be tempting to introduce some kind of shear or velocity gradient 590 threshold to identify when Method A might be preferable, to do so would be misleading as 591 the threshold will also depend on the distance of the shear from the aDcp.

592 "Figure 16"

593 Results also confirm that several repeat transects are indispensable to provide a robust 594 estimation of secondary circulation and to reduce the effect of spatial inhomogeneity and 595 temporal variations. Although Method A reduces the minimum number of repeat transects 596 needed to estimate the secondary velocities, a larger number of these minimum repeat 597 transects (6 or more repeats for Lizerne-Rhône confluence) appeared to be required. This 598 is higher than in the earlier findings of Szupiany et al. (2007) and Vermeulen et al., (2014b) 599 who argue that 5 repeats are enough to have a robust estimation of the turbulence 600 averaged velocity. We also note that an even number of repeats may be important to 601 avoid directional bias in dGPS positions.

602 Since Method A is based on the position of beams, if the bin position errors related to 603 dGPS accuracy as well as sensor tilt are greater than homogeneity errors associated with 604 beam divergence, standard Method $B$ is more reliable. This is likely to be the case 605 particularly in rivers shallower than those studied here and where high resolution is 606 required due to large velocity gradients. In rivers of the scale studied here, and deeper, 607 by increasing the mesh cell size, we can still have sufficient data to estimate velocity 608 vectors, and the effects dGPS and tilt sensor errors have a minor effect. This confirms the 609 earlier findings by Vermeulen et al., (2014b), which showed that Method A provides the 610 greatest improvement where the aDcp cell size is much smaller than the beam spread. 611 We are not yet in a position to identify the depth at which Method A becomes preferable 
612 to Method B, and again this will depend on other parameters such as the intensity of shear 613 and so may not be readily generalizable between confluences.

614 The difficulty of identifying the depths of rivers and intensities of shear that make one 615 method preferable over another precludes adoption of simple quantitative guidance on 616 which method to use when. As both methods have some disadvantages, we argue that 617 both methods should be applied. If they give similar results, then there should be 618 confidence in both. If and where they differ, analysis should be undertaken to identify why, 619 and hence which method is likely to be preferable. Association of the differences in 620 primary and secondary velocities inferred between the two methods with estimates of 621 shear intensity and with estimated tilt and positioning errors should then help decide 622 whether Method A or Method B is preferable in a particular case. This preference may 623 vary between confluences but also through time at a confluence, if shear or flow depth 624 changes significantly between survey dates.

625 Finally, we wish to emphasise that the impact of averaging is only one element that must 626 be considered in obtaining reliable primary and secondary clow estimates in river 627 confluences. Other issues, such as the rotation method needed to distinguish primary and 628 secondary circulation, remain important and should be considered routinely.

\section{Conclusions}

630 This paper shows the advantage of working with the radial (beam) velocity measurements 631 of an aDcp within each bin prior to averaging them across a given volume of fluid (Method 632 A) as opposed to identify volumes of fluid and assuming bend homogeneity within them 633 (Method B). Such a treatment is important where there are strong velocity gradients in the 634 flow as with river channel confluences. In the first of our case-study confluences, the 
635 Lizerne-Rhône, a very small tributary joined the main river, and the pattern of primary and 636 secondary velocities obtained with methods $A$ and $B$ were relatively similar, more so for 637 primary velocities. But for a second confluence, the Grande Eau-Rhône, with a similar 638 momentum ratio, there were much larger differences. We attributed this to the formation 639 of much stronger shear at this confluence. Method A also appeared to reduce the number 640 of repeat transects needed to estimate secondary velocities reliably. The main downside 641 is that Method $A$ is more sensitive to errors related to positioning. Thus, good dGPS 642 accuracy and precision are required to perform a robust estimation of velocity.

643 In smaller/shallower rivers, Method B may be acceptable indeed preferable as it is less 644 sensitive to GPS errors. In larger rivers, Method A may be necessary, especially in the 645 presence of strong shear at the confluence. Choice between these methods should be 646 based upon an initial screening of the extent to which there is strong shear in the flow as 647 well as the extent to which bins further from the aDcp are influenced by beam divergence.

\section{Appendix A}

649 The LOWESS model is a locally weighted polynomial regression, which at each point and 650 in the range of dataset, a low degree polynomial is fitted to a subset of the data, using 651 weighted least squares. This polynomial fit gives more weight to points closer to the point 652 whose response is being estimated. The value of the regression function for the point is 653 then obtained by evaluating the local polynomial using the explanatory variable values for 654 that data point. The LOWESS fit is complete after regression function values have been 655 computed for each of the $n$ data points. Many of the details of this method, such as the 656 degree of the polynomial model and the weights, are flexible ("Local regression," n.d.). 


\section{Acknowledgements}

658 This paper benefited from constructive and detailed suggestions from two anonymous 659 reviewers and Editor Mike Kirkby. The work was supported by Swiss National Science 660 Foundation Grant 200021_160020. The codes used may be obtained upon request to 661 Gelare Moradi.

\section{References}

663 Alvarez, L. V., Schmeeckle, M.W., Grams, P.E., 2017. A detached eddy simulation model 664 for the study of lateral separation zones along a large canyon-bound river. J. Geophys. 665 Res. Earth Surf. 122, 25-49. https://doi.org/10.1002/2016JF003895.

666 Ashmore, P.E., Ferguson, R.I., Prestegaard, K.L., Ashworth, P.J. and Paola, C., 1992. 667 Secondary flow in coarse-grained braided river confluences. Earth Surface Processes 668 and Landforms, 17, 299-312.

669 Best, J.L. and Roy, A.G., 1991. Mixing-layer distortion at the confluence of channels of 670 different depth. Nature, 350, 411-413.

671 Biron, P., De Serres, B., Roy, A.G., Best, J.L., 1993. Shear layer turbulence at an unequal 672 depth channel confluence. In: Clifford, N.J., French, J.R., Hardisty J. (editors) 673 Turbulence: Perspectives on Flow and Sediment Transport, Wiley, Chichester, 197674213.

675 Dinehart, R.L. and Burau, J.R., 2005. Averaged indicators of secondary flow in repeated 676 acoustic Doppler current profiler crossings of bends. Water Resources Research, 41, $677 \quad$ W09405. 
678 Engel, F.L. and Rhoads, B.L., 2016. Three-dimensional flow structure and patterns of bed 679 shear stress in an evolving compound meander bend. Earth Surface Processes and $680 \quad$ Landforms, 41, 1211-1226.

681 Gualtieri, C., lanniruberto, M., Filizola, N., Santos, R. and Endreny, T., 2017. Hydraulic 682 complexity at a large river confluence in the Amazon basin. Ecohydrology, 10, e1863

683 Gunawan, B., Neary, V.S., McNutt, J.R., 2011. Ornl Adv Post-Processing Guide and 684 Matlab Algorithms for Mhk Site Flow and Turbulence Analysis, Ornl/Tm-2011/404. 685 doi:10.2172/1034377.

686 Jackson, P.R., Garcia, C.M., Oberg, K.A., Johnson, K.K. and Garcia, M.H., 2008. Density 687 currents in the Chicago River: Characterization, effects on water quality, and potential 688 sources. Science of the Total Environment 401: 130-143.

689 Kasvi, E., Vaaja, M., Alho, P., Hyyppä, H., Hyyppä, J., Kaartinen, H. and Kukko, A., 2013. 690 Morphological changes on meander point bars associated with flow structure at 691 different discharges. Earth Surface Processes and Landforms, 38, 577-590.

692 Kasvi, E., Laamanen, L., Lotsari, E. and Alho, P., 2017. Flow Patterns and Morphological 693 Changes in a Sandy Meander Bend during a Flood-Spatially and Temporally Intensive 694 ADCP Measurement Approach. Water, 9, 106.

695 Knox, R. L. and Latrubesse, E.M., 2017. A geomorphic approach to the analysis of 696 bedload and bed morphology of the Lower Mississippi River near the Old River Control 697 Structure. Geomorphology, 268, 35-47. 
698 Konsoer, K.M. and Rhoads, B.L., 2014. Spatial-temporal structure of mixing interface 699 turbulence at two large river confluences. Environmental Fluid Dynamics, 14, $1043-$ $700 \quad 1070$.

701 Kostaschuk, R.A., Shugar, D., Best, J.L., Parsons, D.R., Lane, S.N., Hardy, R.J. and 702 Orfeo, O., 2009. Suspended sediment transport and deposition over a dune: Río 703 Paraná, Argentina. Earth Surface Processes and Landforms, 34, 1605-1611.

704 Lane, S.N., Parsons, D.R., Best, J.L., Orfeo, O., Kostaschuk, R. a., Hardy, R.J., 2008. 705 Causes of rapid mixing at a junction of two large rivers: Río Paraná and Río Paraguay, 706 Argentina. Journal of Geophysical Research, 113, 1-16. 707 https://doi.org/10.1029/2006JF000745.

708 Lane, S.N., Bradbrook, K.F., Richards, K.S., Biron, P.M., Roy, a. G., 2000. Secondary 709 circulation cells in river channel confluences : measurement artefacts or coherent flow 710 structures? Hydrological Processes, 14, 2047-2071. https://doi.org/10.1002/1099711 1085(20000815/30)14:11/12<2047::aid-hyp54>3.0.co;2-4.

712 Local regression [WWW Document], n.d.

713 URL:https://en.wikipedia.org/wiki/Local_regression (accessed 1.22.18).

714 Lotsari, E., Kasvi, E., Kämäri, M., and Alho, P., 2017. The effects of ice cover on flow 715 characteristics in a subarctic meandering river. Earth Surface Processes and 716 Landforms, 42, 1195-1212.

717 Marsden, R.F. and Ingram, R.G., 2004. Correcting for beam spread in acoustic Doppler 718 current profiler measurements. Journal of Atmospheric and Oceanice Technology, 21, 719 1491-1498. 
720 Morlock, S.E., 1996. Evaluation of Acoustic Doppler Current Profiler Measurements of

721 River Discharge. U.S. Geological Survey Water Resources Investigations Report 95$722 \quad 4218$.

723 Muste, M., Yu, K. and Spasojevic M. 2004. Practical aspects of ADCP data use for 724 quantification of mean river flow characteristics: Part I: Moving-vessel measurements. 725 Flow Measurement and Instrumentation, 15, 1-16.

726 Parsapour-Moghaddam, P. and Rennie, C.D., 2018. Calibration of a 3D Hydrodynamic 727 meandering river model using fully spatially distributed 3D ADCP velocity data. Journal 728 of Hydraulic Engineering, 144, 04018010.

729 Parsons, D.R., Best, J.L., Orfeo, O., Hardy, R.J., Kostaschuk, R.A. and Lane, S.N., 2005. 730 The morphology and flow fields of three-dimensional dunes, Rio Paraná, Argentina: 731 results from simultaneous multibeam echo sounding and acoustic Doppler current 732 profiling. Journal of Geophysical Research, 110, F04S03.

733 Parsons, D.R., Best, J.L., Lane, S.N, Orfeo, O., Hardy, R.J. and Kostaschuk, R.A., 2007. 734 Form roughness and the absence of secondary flow in a large confluence-diffluence, 735 Rio Paraná, Argentina. Earth Surface Processes and Landforms, 32, 155-162.

736 Parsons, D.R., Jackson, P.R., Czuba, J.A., Engel, F.L., Rhoads, B.L., Oberg, K.A., Best, 737 J.L., Mueller, D.S., Johnson, K.K., Riley, J.D., 2013. Velocity Mapping Toolbox (VMT): 738 A processing and visualization suite for moving-vessel ADCP measurements. Earth 739 Surface Processes and Landforms, 38, 1244-1260. 
740 Petrie, J., Diplas, P., Gutierrez, M. and Nam, S., 2013. Combining fixed- and moving741 vessel acoustic Doppler current profiler measurements for improved characterization 742 of the mean flow in a natural river. Water Resources Research, 49, 5600-5614.

743 Rennie, C.D., Millar, R.G. and Church, M.A., 2002. Measurement of bed load velocity 744 using an acoustic Doppler current profiler. Journal of Hydraulic Engineering - ASCE, $745 \quad 128,473-483$.

746 Rennie, C.D. and Millar, R.G., 2004. Measurement of the spatial distribution of fluvial 747 bedload transport velocity in both sand and gravel. Earth Surface Processes and $748 \quad$ Landforms, 29, 1173-1193.

749 Rennie, C.D., Rainville, F., 2006. Case study of precision of GPS differential correction 750 strategies: influence on aDcp Velocity and discharge estimates. Journal of Hydraulic $751 \quad$ Engineering, 132, 225-234.

752 Rennie, C. D., and Church, M., 2010. Mapping spatial distributions and uncertainty of 753 water and sediment flux in a large gravel bed river reach using an acoustic Doppler 754 current profiler, Journal of Geophysical Research - Earth Surface, 115.

755 Rhoads, B.L. and Kenworthy, S.T., 1995. Field measurements of flow structure at a high756 angle asymmetrical stream confluence. Geomorphology 11, 273-293.

757 Rhoads, B.L. and Kenworthy, S.T., 1998. Time-averaged flow structure in the central 758 region of a stream confluence. Earth Surface Processes and Landforms, 23, 171-191.

759 Rhoads, B.L., and Sukhodolov, A.N., 2001. Field investigation of three-dimensional flow 760 structure at stream confluences: 1 . Thermal mixing and time-averaged velocities. Water $761 \quad$ Resources Research, 27, 2393-410. 
762 Rhoads, B.L., and Sukhodolov, A.N., 2004. Spatial and temporal structure of shear layer 763 turbulence at a stream confluence. Water Resources Research, 40, W06304.

764 Rhoads, B.L., and Sukhodolov, A.N., 2008. Lateral momentum flux and the spatial 765 evolution of flow within a confluence mixing interface. Water Resources Research, 44, 766 W08440.

767 Riley, J.D. and Rhoads, B.L., 2012. Flow structure and channel morphology at a natural 768 confluent meander bend. Geomorphology, 163, 84-98.

769 Riley, J.D., Rhoads, B.L., Parsons, D.R. and Johnson K.K., 2015. Influence of junction 770 angle on three-dimensional flow structure and bed morphology at confluent meander 771 bends during different hydrological conditions. Earth Surface Processes and 772 Landforms, 40, 252-271.

773 Shugar, D.H., Kostaschuk, R., Best, J.L., Parsons, D.R., Lane, S.N., Orfeo, O. and Hardy, 774 R,J., 2010. On the relationship between flow and suspended sediment transport over 775 the crest of a sand dune, Río Paraná, Argentina. Sedimentology 57: 252-272.

776 SonTek YSI, 2000. Acoustic Doppler Profiler (ADP ®) Principles of Operation.

777 SonTek YSI, 2010. RiverSurveyor S5/M9 System Manual 115.

778 Sukhodolov, A.N. and Rhoads, B. L., 2001. Field investigation of three-dimensional flow 779 structure at stream confluences 2. Turbulence. Water Resources Research, 27, 24117802424

781 Sukhodolov, A. N., Krick, J., Sukhodolova, T. A., Cheng, Z., Rhoads, B. L. and 782 Constantinescu, G. S., 2017. Turbulent flow structure at a discordant river confluence: 
783 Asymmetric jet dynamics with implications for channel morphology. Journal of 784 Geophysical Research: Earth Surface, 122(6), 1278-1293. 785 https://doi.org/10.1002/2016JF004126.

786 Szupiany, R.N., Amsler, M.L., Best, J.L. and Parsons, D.R., 2007. Comparison of fixed787 and moving vessel measurements with an aDp in a large river. Journal of Hydraulic $788 \quad$ Engineering, 133, 1299-1309.

789 Szupiany, R.N., Amsler, M.L., Parsons, D.R. and Best, J.,L., 2009. Morphology, flow 790 structure, and suspended bed sediment transport at two large braid-bar confluences. $791 \quad$ Water Resources Research, 45, W05415.

792 Szupiany, R.N., Amsler, M.L., Hernandez, J., Parsons, D.R., Best, J.L., Fornari, E. and 793 Trento, A., 2012. Flow fields, bed shear stresses, and suspended bed sediment 794 dynamics in bifurcations of a large river. Water Resources Research, 48, W11515.

795 Tomas, G., Bleninger, T., Rennie, C.D., Guarneri, H., 2018. Advanced 3D mapping of 796 hydrodynamic parameters for the analysis of complex flow Motions in a submerged 797 bedrock canyon of the Tocantins River, Brazil. Water (Switzerland) 10, 1-19. $798 \quad$ https://doi.org/10.3390/w10040367.

799 Tsubaki, R., Kawahara, Y., Muto, Y. and Fujita, I., 2012. New 3-D flow interpolation 800 method on moving ADCP data. Water Resources Research, 48, 1-15.

801 Venditti, J.G., Rennie, C.D., Bomhof, J., Bradley, R.W., Little, M., Church, M., 2014. Flow 802 in bedrock canyons. Nature 513, 534-537. https://doi.org/10.1038/nature13779. 
803 Vermeulen, B., Hoitink, A.J.F., van Berkum, S.W. and Hidayat, 2014a. Sharp bends 804 associated with deep scours in a tropical river: The river Mahakam (East Kalimantan, 805 Indonesia). Journal of Geophysical Research, Earth Surface, 119, 1441-1454.

806 Vermeulen, B., Sassi, M.G. and Hoitink, A.J.F., 2014b. Improved flow velocity estimates 807 from moving-boat ADCP measurements. Water Resources Research, 50, 4186-4196.

808 Vermeulen, B., Hoitink, A.J.F. and Labeur, R.J., 2015. Flow structure caused by a local 809 cross-sectional area increase and curvature in a sharp river bend. Journal of 810 Geophysical Research, Earth Surface, 120, 1771-1783.

811 Zhao, J., Chen, Z., and Zhang, H., 2014. A robust method for determining the heading 812 misalignment angle of GPS compass in ADCP measurement. Flow Measurements and 813 Instrumentation, 35, 1-10. 